Am. J. Trop. Med. Hyg., 102(Suppl 3), 2020, pp. 234-236

Copyright (C) 2020 by The American Society of Tropical Medicine and Hygiene

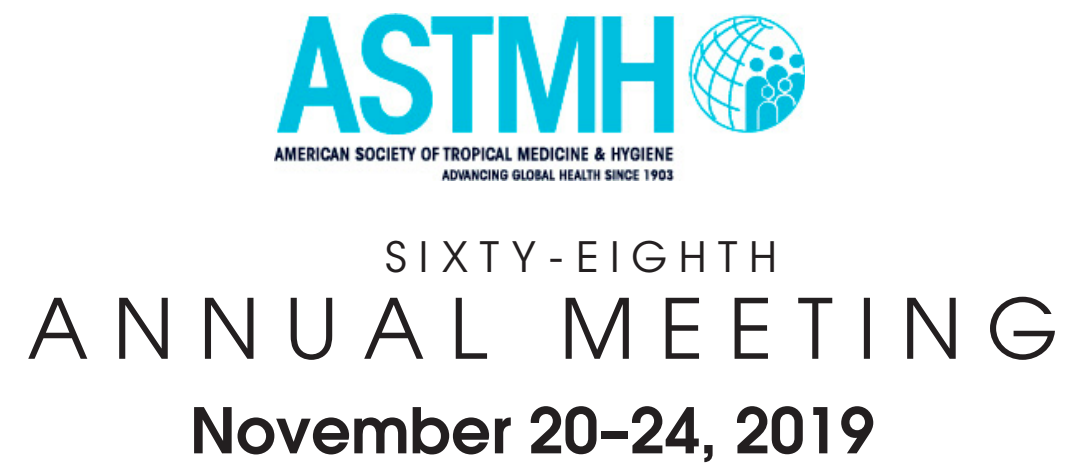

Gaylord National Resort and Convention Center | National Harbor, Maryland

ANNUAL BUSINESS MEETING

November 19, 2019

MINUTES

*DENOTES VOTING MEMBER

2019 Executive Committee

President

Chandy C. John, MD, FASTMH*

President-Elect

Joel G. Breman, MD, DTPH, FASTMH*

Immediate Past President

N. Regina Rabinovich, MD, FASTMH*

Secretary-Treasurer

David R. Hill, MD, DTMH, FASTMH

Scientific Program Chair

Daniel G. Bausch, MD, MPH\&TM, FASTMH

AJTMH Editor-in-Chief

Philip Rosenthal, MD, FASTMH

CEO

Karen A. Goraleski
2019 Board of Directors

Nicole Achee, PhD*

Koya Allen, PhD, MSPH*

Abdoulaye Djimde, PharmD, PhD*

David Fidock, PhD, FASTMH*

David Hamer, MD, FASTMH*

Albert Icksang Ko, MD, FASTMH*

Julie Jacobson, MD*

Miriam Laufer, MD*

Jetsumon Sattabongkot Prachumsri, PhD*

Anna Uehara, PhD, MScGH*

\section{SUBGROUP LEADERS}

American Committee on Arthropod-Borne Viruses (ACAV) Lark Coffey, PhD

American Committee of Medical Entomology (ACME)

Matthew Thomas, PhD

American Committee of Molecular, Cellular and Immunoparasitology (ACMCIP)

Michael Ferdig, PhD

American Committee on Clinical Tropical Medicine and Travelers' Health (ACCTMTH)

M. Patricia Joyce, MD, FIDSA, FASTMH

ASTMH Committee on Global Health (ACGH)

Julie Pavlin, MD, PhD, MPH 


\section{2019- Year in Review}

Karen A. Goraleski acknowledged staff and thanked them for their work and dedication. She reported on the accomplishments of ASTMH over the past year including:

- Inclusion/Respect Policy

- Two Journal supplements

- Selection of the 2019 Alan J. Magill Fellowship recipient Deusdedith Ishengoma, PhD, NIMR-TANGA Centre, Tanzania

- $\quad$ Second Congressional Staff delegation to the CDC in partnership with GHTC, PATH and GHC

- $\quad$ Fourth annual WRAIR Alan Magill Malaria forum

- $\quad$ ASTMH is a member of the National Academies' "Forum on Microbial Threats"

\section{Society Business}

A. Reports from the Board

a. Finances

David R. Hill, MD, DTM\&H, FRCP, FASTMH

reviewed the Society's assets and explained the

difference between restricted and non-restricted funds.

Dr. Hill also reviewed the Society's income sources and expenses.

b. Recognition of Deceased Members

Dr. Hill reviewed the names of those members who have passed away since the last Annual Business Meeting and requested that all stand for a moment of silence.

c. Membership

Dr. Hill presented the membership report. He stated that membership remains steady and reviewed the numbers for each member type. He noted that trainee members often do not transition to full membership, and that we now have an early career membership category to encourage long-term engagement with the Society.

He also reviewed changes to the affiliate membership category the Membership Committee is proposing to the Board of Directors. An outline of the proposed changes of the affiliate category are as follows:

- Universities, university programs, NGOs, professional societies

- Goal is to create a sense of community amongst affiliates, while also providing all membership benefits

- Particularly aimed at trainees

- Broad outline:

o Membership for designated faculty member/representative and 10-15 trainees

O Tiered fee system for LMICs, UMI and HI countries d. Fellows of ASTMH (FASTMH)

Dr. Hill reviewed the 2019 FASTMH and recognized them for their excellence and dedication to the Society.

e. Annual Meeting- Dan Bausch, MD, MPH\&TM, FASTMH reported that the 2019 Annual Meeting had the highest attendance to date with 4,800 registrants (final number to be confirmed after all counts are finalized). Dr. Bausch also reported that in the coming year, the Society will be working with the Digital Education Committee to extend Annual Meeting content beyond the Annual Meeting in an effort to engage persons who were unable to attend.

Dr. Bausch stated that 2020 will be his last year as the Chair of the Scientific Program Committee.

The search for the next Chair will begin after the 2019 Annual Meeting.

f. AJTMH-Philip Rosenthal, MD, FASTMH reviewed highlights from the Society's journal, the American Journal of Tropical Medicine and Hygiene, from the past year. He thanked the Section Editors, Editorial Board and Journal staff, in particular Cathi Siegel, for their hard work and dedication.

B. Subgroup Reports

a. ACAV (American Committee on ArthropodBorne Viruses) - Chair, Lark Coffey, PhD reported on the accomplishments of ACAV from the past year:

- Initiated a strategic plan with a focus on fundraising

- Created a new logo

- $\quad$ Selected a student member dedicated to social media

- $\quad$ Several travel awards were provided to students/trainees

b. ACME (American Committee of Medical Entomology) - Chair-Elect, Ellen Dotson, BS, MS, DSc reported the accomplishments of ACME from the past year:

- $\quad$ Successful speed networking event and student lunch

- $\quad$ Several travel awards were provided to students/trainees

- 2019 Harry Hoogstraal Medal awarded to Frank H. Collins, PhD, University of Notre Dame

c. ACMCIP (American Committee of Molecular, Cellular and Immunoparasitology) President, Michael Ferdig, PhD reported the accomplishments of ACMCIP over the last year:

- $\quad$ Created a new logo and Twitter account

- 2019 William Trager Award awarded to David Horn, PhD, University of Dundee 
- Formed a Student/Trainee Committee

d. ACCTMTH (American Committee on Clinical Tropical Medicine and Travelers' Health) - M. Patricia Joyce, MD, FIDSA, FASTMH reported on the accomplishments of the Clinical Group over the past year:

- Inaugural Martin S. Wolfe Mentoring Award awarded to Elaine Jong, MD, FASTMH, University of Washington School of Medicine

- Hosted a webinar for students on the topic Chagas disease

- Donated funds to the Journal for page waivers

e. ACGH (ASTMH Committee on Global Health)-Julie Pavlin, MD, PhD, MPH President reported the accomplishments over the past year:

- Developing a strategic plan

- Completed a membership survey

- Hosted a webinar for the first time

- Awarded several travel awards

C. Election Results

Dr. Hill reviewed the 2020 election results: Julie Jacobson, MD is the President-Elect; Hanna Ehrlich, PhD; A. Desiree LaBeaud, MD, MS and Katherine Taylor, PhD, MSc have been elected to the Board of Directors. Jonathan Stiles, PhD was appointed by President Chandy John, MD, MS, FASTMH to fulfill Dr. Jacobson's last year as Board member.

\section{Leadership Transition}

A. Recognition of Outgoing Board of Director members

Chandy John, MD, MS, FASTMH thanked the outgoing Board members, Nicole Achee, Koya Allen, David Fidock and Julie Jacobson for their service and gave each a small gift of gratitude.

B. Acknowledgement of Continuing Board Members Dr. John asked the Executive Committee and continuing Board members to stand and be recognized.

C. Transfer of Authority

Dr. John acknowledged and thanked his colleagues, ASTMH and Board members for their support. He then transferred authority to Joel G. Breman, MD, DTPH, FASTMH.

Dr. Breman thanked Dr. John for his service and leadership and presented him with a gift of gratitude. He then thanked Dr. Rabinovich, Dr. Jacobson and Karen Goraleski for their efforts in implementing the inclusion/respect policy and briefly outlined his vision for the next year.

There being no further business, Dr. Hill adjourned the meeting at 1:25 pm EST. 


\section{STATEMENT OF CHANGES IN NET ASSETS FOR THE TWELVE MONTHS ENDING 6/30/19}

\author{
Revenue \\ Unrestricted Revenue \\ 10-Membership \\ 20-Annual Meeting \\ 25-Website \\ 28-Update Course \\ 29-CTropMed Exam \\ 90-Journal Sheridan \\ 91-Journal Ingenta \\ 92-Journal Other \\ 93-ACAV PMC \\ 95-ACCTMTH PMC \\ 96-ACMCIP PMC \\ 97-ACGH PMC \\ 98-ACME PMC \\ Total Unrestricted Revenue \\ Restricted Revenue \\ 31-34 - ACAV \\ 35 - Council Initiatives \\ 30 - Presidents Challenge \\ 40-42 - ACCTMTH \\ 45-ACMCIP \\ 50-52 - ACME \\ $60-\mathrm{ACGH}$ \\ 71-72 - Ben Kean \\ 74-74 - Centenial \\ 78-Shope \\ 79 \& 84 - Magill \\ 80-81 - Fred Soper \\ 83-Commerative Lec \\ 85-BWF Research
}

AMERICAN SOCIETY OF TROPICAL MEDICINE AND HYGIENE
Summary Income Statement

For the Twelve Months Ending Sunday, June 30, 2019

Current Current Fiscal Annual Budget

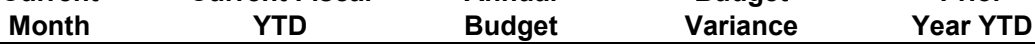

\begin{tabular}{|c|c|c|c|c|}
\hline \multirow[t]{2}{*}{$\$ 43,091$} & $\$ 662,391$ & $\$ 611,175$ & $\$ 51,216$ & $\$ 575,638$ \\
\hline & $3,080,254$ & $2,999,480$ & 80,774 & $2,995,337$ \\
\hline 1,270 & 5,534 & & 5,534 & 9,061 \\
\hline \multirow[t]{2}{*}{2,485} & 24,640 & 60,170 & $(35,530)$ & 13,845 \\
\hline & 79,100 & 82,820 & $(3,720)$ & \\
\hline \multirow[t]{4}{*}{79,136} & 941,343 & $1,010,400$ & $(69,057)$ & $1,151,031$ \\
\hline & 2,247 & 3,500 & $(1,253)$ & 4,475 \\
\hline & 99,842 & 104,500 & $(4,658)$ & 42,954 \\
\hline & & & & 20,480 \\
\hline \multirow[t]{2}{*}{1,655} & 18,135 & 33,585 & $(15,450)$ & 12,635 \\
\hline & 13,445 & 20,860 & $(7,415)$ & 9,350 \\
\hline 300 & 12,355 & 27,125 & $(14,770)$ & 20,785 \\
\hline 150 & 7,875 & 22,050 & $(14,175)$ & \\
\hline 128,087 & $4,947,161$ & $4,975,665$ & $(28,504)$ & $4,855,591$ \\
\hline 848 & 9,406 & 3,375 & 6,031 & 6,276 \\
\hline 63,612 & 63,612 & & 63,612 & \\
\hline 845 & 84,911 & & 84,911 & \\
\hline 8,999 & 31,981 & 13,200 & 18,781 & 24,235 \\
\hline 1,111 & 9,685 & 2,835 & 6,850 & 6,283 \\
\hline 145 & 18,235 & 3,800 & 14,435 & 5,091 \\
\hline 1,182 & 12,518 & 6,700 & 5,818 & 7,026 \\
\hline 64,463 & 99,560 & & 99,560 & 116,607 \\
\hline 69,091 & 104,683 & & 104,683 & 101,790 \\
\hline 65,292 & 96,089 & & 96,089 & 90,651 \\
\hline 99,556 & 188,791 & & 188,791 & 170,605 \\
\hline 1,581 & 3,373 & & 3,373 & 2,863 \\
\hline 451 & 2,637 & & 2,637 & 1,496 \\
\hline 21,058 & 34,359 & & 34,359 & $1,192,810$ \\
\hline
\end{tabular}


AMERICAN SOCIETY OF TROP MED \& HYGIENE

\section{STATEMENT OF CHANGES IN NET ASSETS FOR THE TWELVE MONTHS ENDING 6/30/19}

86-Gates

87-Kazura

Total Restricted Revenue

Total Revenue

\section{Expenses}

Unrestricted Expenses

10-Membership

11-Administration

12-Council

13-Membership/Committee

14-Gov't Relations

15-PR/Media

20-Annual Meeting

25-Website

28-Update Course

29-CTrpoMed Exam

90-Journal Sheridan

91-Journal Ingenta

92-Journal Other

93-ACAV PMC

95-ACCTMTH PMC

96-ACMCIP PMC

97-ACGH PMC

98-ACME PMC

Total Unrestricted Expenses

Restricted Expenses

31-34 - ACAV

40-42 - ACCTMTH

45-ACMCIP

50-52 - ACME

\begin{tabular}{rrrrr}
\multicolumn{1}{c}{$\begin{array}{c}\text { Current } \\
\text { Month }\end{array}$} & $\begin{array}{c}\text { Current Fiscal } \\
\text { YTD }\end{array}$ & $\begin{array}{c}\text { Annual } \\
\text { Budget }\end{array}$ & \multicolumn{1}{c}{$\begin{array}{c}\text { Budget } \\
\text { Variance }\end{array}$} & \multicolumn{1}{c}{$\begin{array}{c}\text { Prior } \\
\text { Year YTD }\end{array}$} \\
\hline 193 & 357,201 & & 357,201 & 1,195 \\
162 & 5,106 & & 5,106 & 1,764 \\
\hline 398,589 & $1,122,147$ & 29,910 & $1,092,237$ & $1,728,692$ \\
\hline & & & & \\
\hline $\mathbf{5 2 6 , 6 7 6}$ & $\mathbf{6 , 0 6 9 , 3 0 8}$ & $\mathbf{5 , 0 0 5 , 5 7 5}$ & $\mathbf{1 , 0 6 3 , 7 3 3}$ & $\mathbf{6 , 5 8 4 , 2 8 3}$ \\
\hline
\end{tabular}

\begin{tabular}{rrrrr}
1,133 & 30,228 & 29,517 & 711 & \\
238,619 & 819,492 & 208,448 & 611,044 & 313,734 \\
13,957 & 425,560 & 336,612 & 88,948 & 389,422 \\
42,027 & 207,451 & 262,813 & $(55,362)$ & 257,394 \\
18,908 & 257,330 & 262,136 & $(4,806)$ & 206,766 \\
18,290 & 326,179 & 287,514 & 38,665 & 209,138 \\
50,402 & $2,437,656$ & $2,573,173$ & $(135,517)$ & $2,409,981$ \\
33,118 & 177,988 & 95,441 & 82,547 & 125,183 \\
52,982 & 87,041 & 72,681 & 14,360 & 73,233 \\
9,176 & 67,795 & 100,871 & $(33,076)$ & 41,051 \\
26,126 & 320,545 & 442,700 & $(122,155)$ & 398,842 \\
9,104 & 46,537 & 43,875 & 2,662 & 36,348 \\
64,605 & 369,094 & 344,797 & 24,297 & 362,470 \\
& & & & 12,051 \\
& 24,526 & 29,155 & $(4,629)$ & 25,025 \\
& 17,823 & 20,605 & $(2,782)$ & 15,586 \\
& 19,735 & 25,955 & $(6,220)$ & 13,790 \\
\hline 578,447 & 8,877 & 21,377 & $(12,500)$ & \\
\hline
\end{tabular}

$\begin{array}{rrrrr} & 534 & 5,673 & (5,140) & 4,003 \\ & 7,146 & 10,908 & (3,762) & 3,802 \\ 108 & 109 & 8,325 & (8,216) & 1,872 \\ 3,000 & 3,000 & 4,080 & (1,080) & 6,240\end{array}$




\section{STATEMENT OF CHANGES IN NET ASSETS FOR THE TWELVE MONTHS ENDING 6/30/19}

\begin{tabular}{|c|c|c|c|c|c|}
\hline & $\begin{array}{l}\text { Current } \\
\text { Month }\end{array}$ & $\begin{array}{c}\text { Current Fiscal } \\
\text { YTD }\end{array}$ & $\begin{array}{l}\text { Annual } \\
\text { Budget }\end{array}$ & $\begin{array}{c}\text { Budget } \\
\text { Variance }\end{array}$ & $\begin{array}{c}\text { Prior } \\
\text { Year YTD } \\
\end{array}$ \\
\hline 60-Global Health Council Designated & & 659 & 3,942 & $(3,283)$ & 685 \\
\hline 71-72 - Ben Kean & 9,011 & 32,625 & 65,718 & $(33,093)$ & 67,518 \\
\hline 74-74 - Centenial & & & 25,300 & $(25,300)$ & 57,975 \\
\hline 78-Shope & & 50,348 & 600 & 49,748 & 7,112 \\
\hline $79 \& 84$ - Magill & $(5,124)$ & 93,849 & 201,680 & $(107,831)$ & 94,478 \\
\hline 80-81 - Fred Soper & & $(376)$ & & $(376)$ & 2,727 \\
\hline 83-Commerative Lec & & 2,554 & 9,256 & $(6,702)$ & 3,082 \\
\hline 85-BWF Research & 130,000 & 525,269 & 195,300 & 329,969 & 174,286 \\
\hline 86-Gates & & & 14,032 & $(14,032)$ & 105,104 \\
\hline 87-Kazura & & & & & 28 \\
\hline Total Restricted Expenses & 136,995 & 715,717 & 544,814 & 170,901 & 528,912 \\
\hline Total Expenses & 715,442 & $6,359,574$ & $5,702,484$ & 657,089 & $5,418,926$ \\
\hline Net Income (Loss) - Unrestricted & $(450,360)$ & $(696,696)$ & $(182,005)$ & $(514,692)$ & $(34,423)$ \\
\hline Net Income (Loss) - Restricted & 261,594 & 406,430 & $(514,904)$ & 921,337 & $1,199,780$ \\
\hline Net Income (Loss) & $(\$ 188,766)$ & $(\$ 290,266)$ & $(\$ 696,909)$ & $\$ 406,645$ & $\$ 1,165,357$ \\
\hline
\end{tabular}


Am. J. Trop. Med. Hyg., 102(Suppl 3), 2020, pp. 240-246

Copyright (C) 2020 by The American Society of Tropical Medicine and Hygiene

\section{CONSTITUTION AND BYLAWS OFTHE AMERICAN SOCIETY OF TROPICAL MEDICINE AND HYGIENE}

Organized 17 November 1951 at Chicago, Illinois Incorporated under the laws of the State of Delaware, 21 January 1952

\section{CONSTITUTION}

\section{NAME}

Section 1.1 Name

The name of the corporation is the "American Society of Tropical Medicine and Hygiene" (the "Society").

\section{ARTICLE II}

\section{REGISTERED OFFICE AND RESIDENT AGENT}

\section{Section 2.1 Registered Office}

This Society shall continuously maintain a registered office in the State of Delaware, as specified in the Articles of Incorporation, which may be, but need not be, the same as its principal office.

\section{Section 2.2 Resident Agent}

The Society shall have a registered agent, as specified in the Articles of Incorporation.

\section{ARTICLE III \\ PURPOSE, POWERS, AND OPERATIONS}

\section{Section 3.1 Purpose and Powers}

The Society is organized exclusively for charitable, educational, and scientific purposes, including, for such purposes, the making of distributions to organizations that qualify as exempt organizations under Section 501(c)(3) of the United States Internal RevenueCode of 1986, as amended (the "Code"), or the corresponding provision of any future United States Internal Revenue law, and the Delaware General Corporation Law (the "DGCL").

In furtherance of its purposes, the Society has adopted the following primary objective:

The advancement of tropical medicine and hygiene, including medicine, nursing, public health, engineering, entomology, parasitology, global health, and allied specialties in this field. In furthering its charitable and educational purposes and fulfilling its mission, the Society shall have the power to do anything else that the Society may be authorized to do under the DGCL and that is consistent with its tax-exempt status under Section 501(c)(3) of the Code, or the corresponding provision of any future United States Internal Revenue law.
No action shall be taken that would adversely affect the qualifi- cations of the Society under Section 501(c)(3) of the Code, or the corresponding provision of any future United States Internal Revenue law.

\section{Section 3.2 Limitations on Powers}

In the course of the operations of the Society: No part of the net earnings of the Society shall inure to the benefit of, or be distributable to, any private shareholder or individual, its directors, officers or other private persons, except that the Society shall be authorized and empowered to pay reasonable compensation for services rendered and to make payments and distributions in furtherance of the purposes set forth herein;

No substantial part of the activities of the Society shall be the carrying on of propaganda, or otherwise attempting to influence legislation, and the Society shall not participate in, or intervene in, (including the publishing or distribution of statements) any political campaign on behalf of, or in opposition to, any candidate for public office, except as authorized under the Code (or any future Internal Revenue law);

Notwithstanding any other provisions of this Constitution and Bylaws, the Society shall not carry on any other activities not permitted to be carried on by (1) a corporation exempt from federal income tax because it is described in Section 501(c) (3) of the Code (or the corresponding provision of any future Internal Revenue law); (2) a corporation, contributions to which are deductible under Section 170(c)(2) of the Code; or (3) a corporation which is not a private foundation under Section 509(a) of the Code.

\section{ARTICLE IV}

\section{MEMBERSHIP}

\section{Section 4.1 Membership}

Members shall have only those rights and obligations set forth in this Constitution and Bylaws.

\section{Section 4.2 Qualifications}

Individuals interested in any phase of tropical medicine, hygiene and related disciplines may apply for Membership in accordance with Section 4.4.

\section{Section 4.3 Classes}

There shall be seven classes of members: Active, Honorary, Life, Emeritus/a, Student, Affiliate, and Fellow. 


\section{ACTIVE MEMBER}

Active Members in good standing pay dues and have the right to vote and hold office. In addition, Active Members shall be granted access to the official publications of the Society, such as the annual volume of The American Journal of Tropical Medicine and Hygiene (the "Journal") and published issues of the Tropical Medicine and Hygiene News (the "News").

\section{HONORARY INTERNATIONAL FELLOW OF ASTMH (FORMERLY KNOWN AS HONORARY MEMBER)}

Any individual not an American citizen who has made eminent contributions to some phase of tropical medicine or hygiene shall be eligible for Honorary International Fellow of ASTMH by election as provided in the Bylaws of the Society. Such Honorary International Fellows of ASTMH shall not vote or pay dues, but shall be given access to the official publications of the Society.

\section{LIFE MEMBER}

Any Active Member or Fellow in good standing may become a Life Member by payment of twenty times the current annual Active or Fellow membership dues, whichever is applicable, in one sum, by which the member becomes exempt from the payment of any future dues assessable during his/her lifetime. Each Life Member shall continue to exercise all rights of an Active Member during his/her lifetime.

\section{EMERITUS/A MEMBER}

Active Members and Fellows in good standing may be transferred to Emeritus/a Membership at their written request to the Secretary-Treasurer. Eligibility consists of 15 continuous years of membership as an Active Member and/or Fellow in good standing and retirement from active work in tropical medicine. Such Emeritus/a Members shall not hold office, vote or pay dues, but will be given access to the official publications of the Society and notices of meetings. Emeritus/a Members may be reinstated as Active or Fellow members upon request and payment of annualdues.

\section{STUDENT MEMBER}

Student Members shall have all the rights and privileges of an Active Member except the right to hold office. Student members include pre-doctoral students and those in postdoctoral fellowships.

Upon graduation or other termination of his/her student status, he/ she may become an Active Member upon payment of Active Member dues. Student status must be certified by the student's department chairperson or major professor upon application, and annuallythere- after upon paying Student Memberdues.

\section{AFFILIATE MEMBER}

Organizations and individuals with demonstrated activity or beneficial interests in the area of tropical medicine may apply to be Affiliate Members.

Each Affiliate member shall appoint one individual as a contact, and that individual may vote and hold office as long as he/she remains the contact person for that Affiliate Member organization. Each Affiliate Member can designate a list of individuals who shall be given access to the official publications of the Society.

\section{FELLOW MEMBER (FASTMH)}

Fellow Member Status (Fellowship) in the Society is an honor recognizing sustained professional excellence in any phase of tropical medicine, hygiene, global health and related disciplines. Any individual with 5 years of continuous Active, Life, or Emeritus/a member- ship in the Society in good standing may be nominated for Fellow Membership on the basis of sustained excellence in investigation or practice. The criteria for nomination and advancement to Fellowship are determined by Council. Applications will be reviewed annually by a Fellowship Committee that will recommend individual members for advancement. Election to Fellowship will occur by majority vote of Council in time to announce new Fellows before the close of the next Annual Business meeting. To maintain Fellowship status, a Fellow Member shall pay annual Society dues until such time as he/she elects to become a Life Member in accordance with the Bylaws. Life Members accepted to Fellow Member status shall continue not to be subject to payment of Society dues.

\section{Section 4.4 Application for Membership}

Application for membership shall be made on the official application form. An application may be rejected by the Secretary-Treasurer should he/she determine it would be in the best interest of the Society to do so. In addition to the categories of individual membership, the Council may create one or more categories within Affiliate Mem- bership and may specify dues for these memberships.

\section{Section 4.5 Membership Dues}

The dues for Active, Fellow, Student and Affiliate Members of the Society shall be determined by the Council. Changes in the dues shall require approval by two-thirds of the Council. Dues shall become due and payable the first day of January of each year.

Any Active, Fellow or Student member who previously paid dues and whose dues payment is in arrears for more than one year shall be dropped from membership. Any Affiliate Member whose dues payment is in arrears for more than three months shall be dropped from membership. 


\section{Section 4.6 Annual Meeting}

The Society shall hold an annual meeting for the conduct of business and scientific sessions unless the Council orders otherwise. The Scientific Program Chair, with the approval of the Council, shall determine the time and place for the annual meeting.

An annual business meeting session shall be held during the annual meeting, and no other event shall be scheduled for the same time.

The minutes of the annual business meeting shall be published in the Journal or the News or other appropriate venue.

In the conduct of business sessions, the current version of Robert's Rules of Order shall be the guide, and at the beginning of each session the President shall appoint a member to serve as Parliamentarian.

\section{Section 4.7 Voting}

Voting of the Membership may be either (a) in person, (b) via mailed or electronically transferred ballots, and (c) via both/either mechanisms. The Active, Life, Student, Affiliate, and Fellow Members (the "Voting Members") attending the annual business meeting, or any other meeting, or responding to a ballot request shall constitute a quorum. Any action of the Members shall be taken by the affirmative vote of the majority of the Voting Members present and voting at such meeting or responding to a ballot request. A Voting Member shall be considered present if he/she either attends the meeting in person or returns a ballot in accordance with the requirements for ballots set forth in Section 4.8.

\section{Section 4.8 Ballots}

For votes to be performed via mailed or electronically transferred ballots, at least two months prior to the annual business meeting, or any other vote to be taken by the Voting Members on matters determined by Council, the Secretary-Treasurer shall send a ballot to each Voting Member of the Society who is in good standing. To be valid, a ballot must be received either via mail or electronic transmission by the Secretary-Treasurer or his/her designee at least two weeks prior to the applicable meeting, so that the Secretary-Treasurer or his/her designee can count the votes and announce the results of the election at the annual business meeting. The timing requirements set forth in this paragraph aremandatory.

\section{Section 4.9 Tie Votes}

In the event balloting as prescribed in section 4.8 results in a tie vote between the top two candidates for any position, the tie will be broken by coin toss. The Secretary-Treasurer, having designated one side of the coin to each candidate in advance, shall conduct the toss.

\section{OFFICERS \\ Section 5.1 Designation of Elected Officers}

The elected Officers of the Society shall be a President, a President-Elect, an Immediate Past President and eight additional Councilors. The elected officers may also be referred to herein as the "Councilors."

\section{Section 5.2 Qualifications}

Any member in good standing and whose membership category permits holding office in the Society is eligible for election to serve as either an elected or appointed officer.

\section{Section 5.3 Designation of Appointed Officers}

A Secretary-Treasurer, a Scientific Program Chairperson, the Editor of the Journal, the Editor of the News, and the Editor of the Web Site all are Officers appointed by the Council. These appointed

Officers are ex-officio, non-voting members of the Council; however, they may make and second motions.

\section{Section 5.4 Election/Appointment of Officers and Term of Office}

The President-Elect shall be elected by the Voting Members in good standing of the Society for a term of one year or until the President-Elect's successor is elected. At the end of the term, the President-Elect shall become the President of the Society for the ensuing year and at the completion of that term will serve for one year as Immediate Past President.

The Secretary-Treasurer, Scientific Program Chairperson, Editor of the Journal, Editor of the News, and Editor of the Web Site shall be appointed by the Council to serve a threeyear term, renewable.

Two Councilors shall be elected annually by the Voting Members in good standing of the Society to serve a four -year term.

Terms of office in the Society shall begin at the close of the business meeting at the annual meeting at which the officers were elected and appointed, except that the SecretaryTreasurer's term of office shall begin on the first day of the Society's next fiscal year following the annual meeting at which the Secretary-Treasurer was appointed.

\section{Section 5.5 Removal and Resignation}

Any officer, whether elected or appointed, may be removed, with or without cause, by a vote of two-thirds of the Voting Members of the Council then in office or two-thirds of the Voting Members in good standing of the Society, whenever in their judgment the best interests of the Society will be served. 


\section{Section 5.6 Vacancies}

If the Presidency becomes vacant, the President-Elect shall become President for the remainder of the unexpired term, and shall then continue in office as President for the full year for which he/she was elected. In the event the PresidentElect is unable to serve, the office shall be filled by the candidate who received the next larger number of votes for President-Elect in the last election, for the remainder of the unexpired term as President-Elect, in which event the offices of President and President-Elect shall be filled in the next election.

The President may fill vacancies among the other Councilors occurring during the year by appointing Councilors to serve until the next annual meeting, when new Councilors are elected.

The Council may fill vacancies among the appointedoffices occurring during the year by naming replacement officers to serve until the next annual meeting, when new appointed officers are designated.

\section{Section 5.7 Duties of the President}

The duties of the President shall be those regularly exercised by the chief executive officer. The President shall preside at meetings of the Council and the Society, appoint committees as provided in the Bylaws, and shall deliver an address at the annual meeting.

\section{Section 5.8 Duties of the President-Elect}

The President-Elect shall succeed the President at the annual meeting except as noted in Section 5.6. The President-Elect shall preside at meetings of the Society in the absence of the President.

\section{Section 5.9 Duties of the Scientific Program Chairperson} The Scientific Program Chairperson shall be responsible for the structure of scientific sessions at the annual meeting. The Scientific Program Chairperson shall be the Chair of the Scientific Program Committee and shall appoint members to the Scientific Program Committee in accordance with Section 7.8 .

\section{Section 5.10 Duties of the Secretary-Treasurer}

The Secretary-Treasurer shall be the custodian of all records of the Society. The Secretary-Treasurer shall handle the business and finances of the Society, including those of Society publications, and shall submit an annual financial report reviewed by a certified public accountant. The Secretary-Treasurer shall be responsible for the arrangement of meetings. The Secretary-Treasurer shall attend and provide for the recording of the minutes of meetings.

\section{Section 5.11 Duties of the Editor of the Journal}

The Editor of the Journal, with the aid of the Editorial Board, is responsible for the form and content of the Journal within the limits and directives established by the Council.

\section{Section 5.12 Duties of the Editor of the News}

The Editor of the News is responsible for the form and content of the News within the limits and directives established by the Council.

\section{Section 5.13 Duties of the Editor of the Web Site}

The Editor of the Web Site is responsible for the form and content of the Web Site within the limits and directives established by the Council.

COUNCIL

\section{ARTICLE VI}

\section{Section 6.1 Membership}

The President, President-Elect, Immediate Past President, and eight Additional Councilors shall constitute the Council.

\section{Section 6.2 Power and Responsibility}

The Council shall manage the Society's affairs and direct its busi- ness. It shall initiate, coordinate, and terminate the work of the committees. It shall appoint the SecretaryTreasurer, the Scientific Program Chairperson, the Editor of the Journal, the Editor of the News, and the Editor of the Web Site. It shall have the power to approve the expenditure of funds and establish an annual budget for the Society. The Council may appoint an Executive Director to assist in the administration of the affairs of the Society. The Executive Director shall have the duties and authority specified by the Council. The Council may delegate to the Executive Director any duties that are delegable under this Constitution and Bylaws and section 141(c)(1) of the Delaware General Corporation Law or any other provision of the DGCL.

\section{Section 6.3 Quorum}

At any meeting of the Council, either regular or special, the presence of a majority of the voting members of the Council shall constitute a quorum.

\section{Section 6.4 Majority Action as Board Action}

Unless otherwise required by the State of Delaware, the Articles of Incorporation, or this Constitution or Bylaws, the vote of a majority of the Councilors present and voting at a meeting at which a quorum is present shall be necessary for the adoption of any matter.

\section{Section 6.5 Telephone/Videoconference Meetings}

Members of the Council may participate in any meeting by means of conference telephone, videoconference, or similar equipment by means of which all persons participating can hear one another in real time. 


\section{Section 6.6 Informal Action}

Any action taken at a meeting of the Council may, with unanimous written consent, be taken by electronic transmission. The written consent action, in the form of an e-mail, text, or other electronic communication which identifies the sender and date and time of the transmission, shall be filed with the minutes of the meeting.

\section{Section 6.7 Chapters of the Society}

The Council shall have the authority to establish affiliated groups as Chapters of the Society upon receipt of a written petition signed by five (5) or more persons who reside in an area where no Chapter exists. Each such Chapter shall function under the provisions of a standard charter which defines the general organizational structure and purposes of the Chapter. There shall be provisions in each Chapter's charter to the effect that:

a) The objectives and purposes of the Chapter are compatible with those of the Society.

b) The President and the President-Elect of the chapter shall be persons who are members in good standing of the Society.

c) Any person who is a member of the Society becomes a member of any class of the Chapter upon presenting proof of membership in the Society and the payment of dues appropriate to the membership class sought in the chapter.

Chapters shall be established as separate legal entities and the Society shall not be responsible for debts or obligations of Chapters.

\section{COMMITTEES}

\section{ARTICLE VII}

\section{Section 7.1 Executive Committee}

The Council shall establish an Executive Committee, which shall exercise the authority of the Council and the management of the Society between meetings of the Council, except as otherwise prohibited by Section 141(c)

(1) of the $\mathrm{DGCL}$ or any other provision of the $\mathrm{DGCL}$, which prohibits a committee from amending the certificate of incorporation, adopting an agreement of merger or consolidation, or amending the Constitution and Bylaws. The Executive Committee shall be composed of the President, the President-Elect, the Immediate Past President, the Secretary-Treasurer, the Editor of the Journal, the Scientific Program Chairperson, and the Executive Director. The Secretary-Treasurer, The Editor of the Journal, the Scientific Program Chairperson and the Executive Director shall participate as non-voting members of the Executive Committee. The duties of the Executive Committee will be as specified and authorized by the Council and may include administrative and management decisions during the course of the year and authorizations of expenditures within guidelines established by the Council.

\section{Section 7.2 Nominating Committee}

The Nominating Committee is charged with developing the slate of candidates for annual Council elections as described below.

The Nominating Committee will be composed of twelve Members of the Society in good standing who represent, insofar as possible, the various scientific disciplines of the Society. Members of the Nominating Committee will be appointed for a two-year term. Half of the new members of the Nominating Committee will be appointed on or before January 1 annually and these members will include the immediate Past-President, the two outgoing Council members from the previous year and three additional members appointed by the President, who also may appoint Members of the Society in good standing to fill any other vacancies on the Committee.

Each immediate Past-President appointed to this Committee will, during the second year of his/her term of appointment, serve as its Chairperson. In the event this individual is unable to serve as Chairperson, the President will appoint another current or past member of the Committee to this position.

At least four months before the next annual meeting the Nominating Committee shall submit to the SecretaryTreasurer the names of two nominees for each elected officer position, with the proviso that nominees should reflect the diversity of the Society membership, including with respect to age, gender, and professional and scientific interests and expertise. The timing requirements set forth in this paragraph are guidelines only, and the failure to rigidly adhere to them shall not invalidate an election.

\section{Section 7.3 Membership Committee}

The Membership Committee supports the SecretaryTreasurer in planning and oversight of the Society's membership recruitment and retention initiatives. The President shall appoint annually a new member with a three-year term to such Membership Committee. The Membership Committee shall be composed of at least three members with staggered terms.

\section{Section 7.4 Honorary International Fellow of ASTMH Membership Committee}

The Honorary International Fellow of ASTMH Membership Committee is charged with nominating potential Honorary International Fellow of ASTMH Members for consideration by Council.

The President shall appoint annually a new member with a three-year term to such Honorary International Fellow of ASTMH Membership Committee. The HonoraryInternational Fellow ofASTMHMembership Committee shall be composed of three members with staggered terms. The Honorary 
International Fellow of ASTMH Membership Committee shall nominate potential Honorary International Fellow of ASTMH Members, who are then elected by unanimous vote of the Council. The Honorary International Fellow of ASTMH Membership Committee shall provide the Council with curricula vitae and summaries of the scientific qualifications of the candidates whom it recommends. No more than five Honorary International Fellow of ASTMH Members may be elected in any one year.

\section{Section 7.5 Scientific Program Committee}

The Scientific Program Committee, under the direction of the Scientific Program Chair, oversees content development for the Society's annual scientific meeting.

The Scientific Program Committee shall be comprised of the Scientific Program Chairperson and additional members as needed. The Scientific Program Chairperson shall appoint members to the Scientific Program Committee for threeyear terms, renewable. Due consideration will be given so that the appointees represent different sub-disciplines of tropical medicine.

\section{Section 7.6 Finance Committee}

The Finance Committee, under the direction of the Secretary-Treasurer, monitors the Society's finances and advises the Council on fiduciary matters.

The Finance Committee shall be comprised of the SecretaryTreasurer, acting as chairperson, the Executive Director, the President-Elect and Additional members as needed.

\section{Section 7.7 Committee on Awards of the Society}

The Committee on Awards of the Society administers the Society's awards and medals, including the Walter Reed Medal, the Joseph Augustin LePrince Medal, the Ben Kean Medal, the Donald Mackay Medal, and the Bailey K. Ashford Medal.

The Council shall appoint the Committee on Awards of the Society consisting of three members, appointing one PastPresident each year for a term of three years; the Chair of the Committee shall be the member with longest seniority on the Committee.

\section{Section 7.8 Other Committees}

The Council is responsible for creating, appointing, and discontinuing other committees required to transact the business of the Society.

\section{ARTICLE VIII \\ PUBLICATIONS OF THE SOCIETY Section 8.1 The American Journal of Tropical Medicine and Hygiene}

The Society may publish the Journal, as the Council deems to be in the best interests of the Society from time to time.
The Editorial Board shall be composed of Members of the Society in good standing to be selected and changed by the Editor, subject to approval by the Council.

\section{Section 8.2 Tropical Medicine and Hygiene News}

The Society may publish the News, as the Council deems to be in the best interests of the Society from time totime.

\section{INDEMNIFICATION}

\section{ARTICLE IX}

\section{Section 9.1 Indemnification}

Any person who was or is a party or is threatened to be made a party to any threatened, pending, or completed action, suit or proceeding, whether civil, criminal, administrative or investigative, by reason of the fact that such person is or was a Councilor or officer of the Society, or is or was serving while a Councilor or officer of the Society at the request of the Society as a director, officer, employee, agent, fiduciary or other representative of another corporation, partnership, joint venture, trust, employee benefit plan or other enterprise, shall be indemnified by the Society against expenses (including attorneys' fees), judgments, fines, excise taxes and amounts paid in settlement actually and reasonably incurred by such person in connection with such action, suit or proceeding to the full extent permissible under Delaware law, except that the Society shall not indemnify such individuals for willful misconduct.

\section{Section 9.2 Advances}

Any person claiming indemnification within the scope of Section 9.1 shall be entitled to advances from the Corporation for payment of the expenses of defending actions against such person in the manner and to the full extent permissible under Delaware law.

\section{Section 9.3 Procedure}

On the request of any person requesting indemnification under Section 9.1, the Council or a committee thereof shall determine whether such indemnification is permissible. If the Council or committee so directs or if the Council or committee is not empowered by statute to make such determination, then such determination shall be made by independent legal counsel.

\section{Section 9.4 Other Rights}

The indemnification and advancement of expenses provided by this Article 9 shall not be deemed exclusive of any other rights to which those seeking indemnification or advancement of expenses may be entitled under any insurance or other agreement, vote of the Council or otherwise, both as to actions in their official capacity and as to actions in another capacity while holding an office, and shall continue as to a person who has ceased to be a Councilor or officer and shall inure to the benefit of the heirs, executors, and administrators of such person. 


\section{Section 9.5 Insurance}

The Society shall have power to purchase and maintain insurance on behalf of any person who is or was a Councilor, officer,employee or agent of the Society or is or was serving at the request of the Society as a director, officer, employee, agent, fiduciary or other representative of another corporation, partnership, joint venture, trust, employee benefit plan or other enterprise, against any liability asserted against him and incurred by him in any such capacity, or arising out of his status as such, whether or not the Society would have the power to indemnify him against such liability under the provisions of this Constitution and Bylaws.

\section{Section 9.6 Modification}

The duties of the Society to indemnify and to advance expenses to a Councilor or officer provided in this Article 9 shall be in the nature of a contract between the Society and each such Councilor or officer, and no amendment or repeal of any provision of this Article 9 shall alter, to the detriment of such Councilor or officer, the right of such person to the advancement of expenses or indemnification related to a claim based on an act or failure to act which took place prior to such amendment, repeal or termination.

\section{ARTICLE $X$ \\ AMENDMENT \\ Section 10.1 Amendment}

The Constitution and Bylaws may be amended at the annual busi- ness meeting by (i) a two-thirds vote of the Voting Members of the Society present and voting, or (ii) at the Council's option, by a favorable two-thirds vote of the Voting Members of the Society who submit a ballot. In either case, such amendments shall have been sent to the SecretaryTreasurer for circulation to the Council at least three months before the meeting. Results of ballots shall be announced at the annual business meeting. 


\section{Executive Committee}

*DENOTES VOTING MEMBER

\section{President}

Joel G. Breman, MD, DTPH, FASTMH*

President-Elect

Julie Jacobson, MD*

Immediate Past President

Chandy C. John, MD, FASTMH*

Secretary-Treasurer

David R. Hill, MD, DTMH, FASTMH
Scientific Program Chair

Daniel G. Bausch, MD, MPH\&TM, FASTMH

\section{AJTMH Editor-in-Chief}

Philip Rosenthal, MD, FASTMH

CEO

Karen A. Goraleski

\section{Board of Directors}

Abdoulaye Djimde, PharmD, PhD*

Hanna Ehrlich, PhD (Candidate)*

David Hamer, MD, FASTMH*

Albert Icksang Ko, MD, FASTMH*

Desiree LaBeaud, MD, MS*
Miriam Laufer, MD*

Jetsumon Sattabongkot Prachumsri, PhD*

Jonathan K. Stiles, PhD*

Katherine Taylor, PhD*

Anna Uehara, PhD, MScGH*

\section{Subgroup Leaders}

$\begin{array}{ll}\text { American Committee on Arthropod-Borne Viruses (ACAV) } & \text { American Committee on Clinical Tropical Medicine and } \\ \text { David M. Morens, AB, MD, FASTMH } & \text { Travelers' Health (ACCTMTH) } \\ & \text { Latha Rajan, MD, MPHTM, FASTMH }\end{array}$

American Committee of Medical Entomology (ACME)

Ellen M. Dotson, MS, DSc

ASTMH Committee on Global Health (ACGH)

Robert D. Newman, MD, MPH

American Committee of Molecular, Cellular and Immunoparasitology (ACMCIP)

Michael Ferdig, PhD

ASTMH Organizational Chart

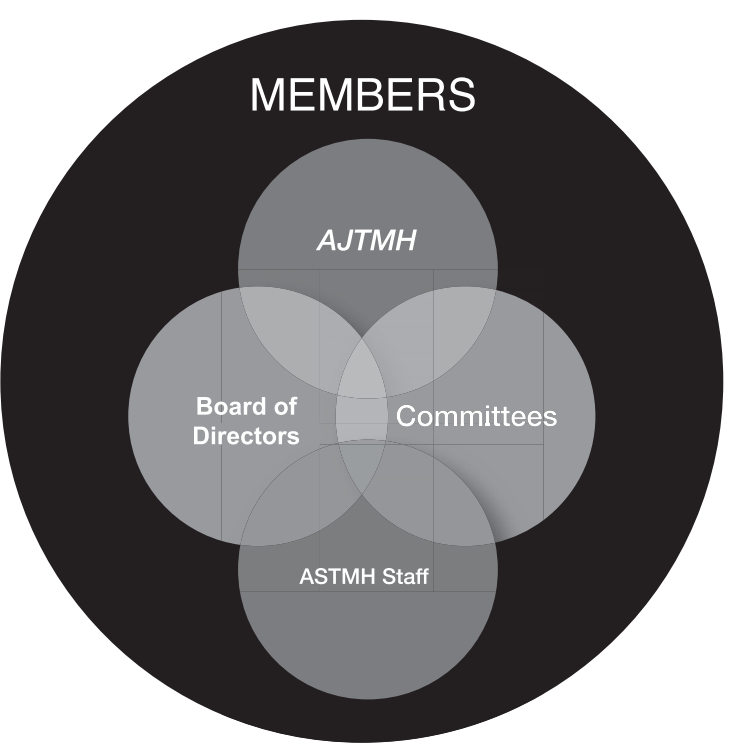




\section{Former Presidents of the Parent Societies}

\section{The National Malaria Society}

*W.H.W. Kemp . . . . . . . 1951-1952

*Justin M. Andrews . . . 1950-1951

* Paul F. Russell . . . . . . . 1949-1950

*Wendell Gingrich . . . . . 1948-1949

*E. Harold Hinman . . . . . 1947-1948

*Mark D. Hollis . . . . . . . . 1946-1947

*Mark F. Boyd . . . . . . . . 1945-1946

*H. A. Johnson . . . . . . . 1944-1945

*George H. Bradley. . . . . 1943-1944

*James S. Simmons . . . . 1942-1943

*John H. O’Neill . . . . . . . 1941-1942

\section{The American Society of Tropical Medicine}

*William A. Sodeman . . 1951-1952 *Paul F. Russell . . . . . . . . 1950-1951

*Hamilton H. Anderson . 1949-1950

*Norman H. Topping . . . 1948-1949

*Joseph S. D’Antoni . . . 1947-1948

*Edward J. Salisbury . . . 1946-1947

*James S. Simmons . . . . 1945-1946

* Rolla E. Dyer . . . . . . . . 1944-1945

*Wilbur A. Sawyer . . . . 1943-1944

*N. Paul Hudson . . . . . . . 1942-1943

*Ernest Carroll Faust . . . 1941-1942

*Thomas T. Mackie. . . . . 1940-1941

*Louis L. Williams . . . . . . 1939-1940

*Alfred C. Reed . . . . . . . . 1938-1939

*Mark F. Boyd . . . . . . . . 1937-1938

*Herbert C. Clark ... . . . 1936-1937

*Henry E. Meleney. . . . . 1935-1936

*Edward B. Vedder . . . . 1934-1935

*Frederick F. Russell . . . 1933-1934

*George R. Callender . . 1932-1933

*Frank Smithies. . . . . . . 1931-1932

*Sidney K. Simon ... . . . 1930-1931

*Kenneth M. Lynch. . . . . 1929-1930

*William E. Deeks . . . . . 1928-1929

*Charles C. Butler . . . . . 1927-1928

*Joseph E. Siler . . . . . . . 1925-1926

*Samuel T. Darling . . . . . 1924-1925

*Allen J. Smith. . . . . . . . 1923-1924

* George Dock . . . . . . . . . 1922-1923

*Victor G. Heiser. . . . . . . 1921-1922

*John M. Swan . . . . . . . 1920-1921

*Henry J. Nichols . . . . . 1919-1920

*Charles C. Bass . . . . . . 1917-1919

*Bailey K. Ashford. . . . . . 1916-1917

*Milton J. Rosenau . . . 1915-1916

*Charles F. Craig . . . . . . 1914-1915

*Richard P. Strong. . . . . 1913-1914

*Edward R. Stitt. . . . . . . 1912-1913

*Joseph H. White . . . . . 1911-1912
*William S. Thayer . . . . 1910-1911

*Willaim C. Gorgas . . . . 1909-1910

*James G. Anders . . . . . . 1908-1909

*Roland G. Curtis . . . . . 1905-1907

*Thomas H. Fenton . . . 1904-1905

\section{The American Society of Tropical Medicine and Hygiene}

Chandy C. John . . . . . . . . . 2019

N. Regina Rabinovich . . . . . 2018

Patricia F. Walker. . . . . . . . . 2017

Stephen Higgs . . . . . . . . . . . 2016

Christopher Plowe . . . . . . . 2015

*Alan J. Magill . . . . . . . . . . . 2014

David H. Walker. . . . . . . . . . 2013

James W. Kazura . . . . . . . . . 2012

Peter J. Hotez . . . . . . . . . . 2011

Edward T. Ryan . . . . . . . . . . 2010

Thomas Wellems ... . . . . . . 2009

Claire Panosian . . . . . . . . . 2008

Carlos C. Campbell . . . . . . . 2007

Myron M. Levine. . . . . . . . . . 2006

Thomas P. Monath . . . . . . . 2005

Peter F. Weller. . . . . . . . . . . 2004

William A. Petri . . . . . . . . . . 2003

Michele Barry . . . . . . . . . . . . 2002

Stephen L. Hoffman . . . . . . . 2001

Duane J. Gubler. . . . . . . . . . . 2000

Dyann Wirth . . . . . . . . . . . 1999

Stephanie James. . . . . . . . . . 1998

Richard L. Guerrant. . . . . . . . 1997

Donald S. Burke . . . . . . . . 1996

Carole A. Long . . . . . . . . . . . 1995

Barnett L. Cline . . . . . . . . . . . 1994

Daniel G. Colley. . . . . . . . . . . 1993

Donald J. Krogstad . . . . . . . . 1992

Scott B. Halstead. . . . . . . . . 1991

John R. David. . . . . . . . . . . . 1990

Louis H. Miller. . . . . . . . . . . 1989

*Jacob K. Frenkel . . . . . . . . 1988

Joseph A. Cook . . . . . . . . . 1987

*Franz von Lichtenberg . . . . 1986

* Paul P. Weinstein. . . . . . . . . 1985

Karl M. Johnson . . . . . . . . 1984

Philip K. Russell . . . . . . . . . . 1983

*Mark T. Hoekenga. . . . . . . . 1982

*William F. Scherer . . . . . . . 1981

* Robert E. Shope . . . . . . . . 1980

*William Trager. . . . . . . . . . 1979

Irving G. Kagan . . . . . . . . . 1978

*Wilford S. Bailey . . . . . . . . 1977

*Leon Rosen . . . . . . . . . . . . 1976

*Geoffrey M. Jeffrey. . . . . . . 1975

* Lloyd E. Rozeboom . . . . . . . 1974

*Harold W. Brown. . . . . . . . 1973

* Paul H. Thompson ... . . . 1972

*William C. Reeves . . . . . . . 1971

*Telford H. Work. . . . . . . . . 1970

* Paul C. Beaver . . . . . . . . . . 1969

*William McD. Hammon . . . 1968
*Harry Most . . . . . . . . . . 1967

* John E. Larsh, Jr. . . . . . . . . 1966

*E. Harold Hinman . . . . . . . 1965

*Thomas H. Weller . . . . . . . 1964

*Clay G. Huff . . . . . . . . . . . . 1963

*G. Robert Coatney . . . . . . 1962

*William W. Frye. . . . . . . . . 1961

*Willard H. Wright . . . . . . . 1960

*Lewis W. Hackett. . . . . . . . 1959

* Donald L. Augustine . . . . . . 1958

*Justin M. Andrews . . . . . . 1957

*Asa C. Chandler . . . . . . . . 1956

*William H. Taliaferro . . . . . . 1955

*Frederick J. Brady . . . . . . . . 1954

*William A. Sodeman . . . . . 1953

* Martin D. Young . . . . . . . . 1952

* Deceased

\section{Past and Present Executive Committee Members}

Secretary-Treasurer-ASTMH
$2012-\ldots . \ldots$. . . David R. Hill
2009-2011 . . . . Jonathan Berman
2003-2008 . . . . George Hillyer
1992-2002 . . . . Peter Weller
1990-1991 . . . . Jonathan I. Ravdin
1988-1989 . . . . William A.
1981-1987 . . . . John E. Scanlon
1976-1980 . . . . Mark T. Hoekenga
1971-1975 . . . . George R. Healy
1968-1970 . . . . Marion M. Brooke
1962-1967 . . . . Geoffrey M. Jeffery
1961. . . . . . . . Don E. Eyles
1957-1960 . . . . Rolla B. Hill
1953-1956 . . . . John E. Larsh, Jr.
1952 . . . . . . . Q Quentin M. Geiman

\section{Secretary-Treasurer-ASTM*}

1950-1951 . . . . . Quentin M. Geiman 1948-1949 ...... Frederick J. Brady

1946-1947 . . . . . Norman H. Topping 1943-1945 ..... . Joseph S. D'Antoni 1941-1942 ... . . . E. Harold Hinman *American Society of Tropical Medicine 


\section{Former Presidents of the Parent Societies (cont.)}

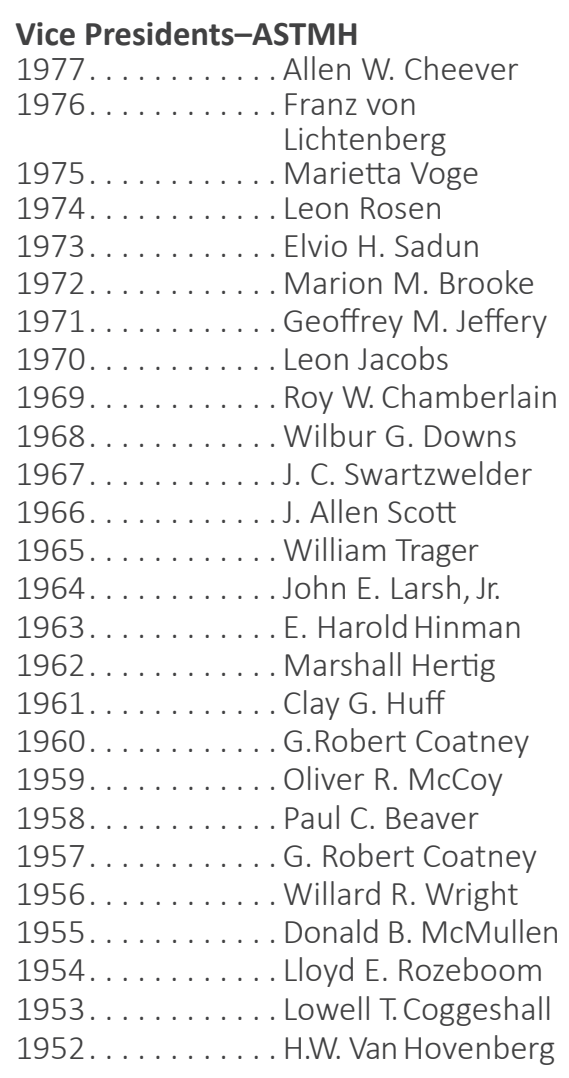

\section{Vice Presidents-ASTM*}

1951. . . . . . . . . Harry Most 1950. . . . . . . . . Frederick J. Brady

1949. . . . . . . . . . J.J. Sapero

1948. . . . . . . . . E.G. Hakansson

1947. . . . . . . . . L.T. Coggeshall

1946. . . . . . . . . J.S. D'Antoni

1945. . . . . . . . . Paul F. Russell

1944. . . . . . . . R.E. Dyer

1943 . . . . . . . . . William A. Sawyer

1942 . . . . . . . . . Fred L. Soper

1941 ... . . . . . . . Fred L. Soper

*American Society of Tropical Medicine
Scientific Program Chairs-ASTMH

2015- . . . . . . . . . Daniel G. Bausch

2009-2014 ... . . Christopher King

2002-2008 . . . . . Edward T. Ryan

1993-2001 ... . . William A. Petri

1990-1992 ... . . . Thomas P. Monath

1984-1989 . . . . . Donald J. Krogstad

1981-1983 ... . . Gordon Wallace

1978-1980 ... . . . McWilson Warren

\section{AJTMH Editor-in-Chief}

2014-. . . . . . . . Philip Rosenthal

2013 . . . . . . . . . . James W. Kazura

2011-2012 . . . . . Joseph M. Vinetz

1998-2011 . . . . . James W. Kazura

1991-1997 . . . . . McWilson Warren

1985-1990 ... . . . W.D. Tigertt

1972-1984 ... . . Paul C. Beaver

1967-1971 . . . . . Robert Briggs Watson

1960-1966 . . . . . Paul C. Beaver

1958-1959 ... . . Martin Frobisher

1952-1957 . . . . . L.W. Hackett

\section{Editor-ASTM* Journal}

1951. . . . . . . . . L.W. Hackett

1947-1950 . . . . . Mark F. Boyd

1941-1946 ... . . Charles F. Craig

*American Society of Tropical Medicine

\author{
Editor-ASTMH News \\ 2006-2010 ... . * *William Collins \\ *Geoffrey Jeffery \\ 2005. . . . . . . . . Michael R. \\ Hollingdale \\ 2000-2004 . . . . . Kathryn S. Aultman \\ 1987-1999 . . . . . Karl A. Western \\ 1967-1986 . . . . . Colvin Gibson \\ 1961-1966 . . . . . William B. DeWitt \\ 1956-1960 . . . . . L Louis J. Olivier \\ 1952-1955 . . . . . . Leon Jacobs \\ *Deceased
}

Editor-ASTM* News

1948-1951 . . . . . . Frederick J. Brody

1946-1947 . . . . . Norman Topping

1944-1945 . . . . . . Joseph S. D'Antoni

*American Society of Tropical Medicine

\section{Editor-Website-ASTMH}

2009-2011 . . . . . Jonathan Mayer

1999-2008 ... . . * *John Woodall

* Deceased

Council Advisor-ASTMH

2006-2014 ... . . Peter F. Weller

Board Advisor-ASTMH

2019-. . . . . . . . Patricia F. Walker 


\section{BOARD OF DIRECTORS (formerly known as Councilors), 1952-2020}

Board of Directo
$\mathbf{2 0 2 0}$
A. Djimde
D. Hamer
J. Sattabongkot
Prachumsri
A. Uehara
A. Ko
M. Laufer
D. LaBeaud
J. Stiles
K. Taylor
H. Ehrlich
2019
N. Achee
D. Fidock
K. Allen
A. Djimde
J. Jacobson
D. Hamer
J. Sattabongkot
Prachumsri
A. Uehara
A. Ko
M. Laufer

\section{Councilors}

2018

L. Kramer

A. Powers

N. Achee

D. Fidock

A. Djimde

J. Jacobson

D. Hamer

J. Settabongkot

Prachumsri

\section{7}

S. Aksoy

L. Slutsker

L. Kramer

A. Powers

N. Achee

D. Fidock

A. Djimde

J. Jacobson

\section{6}

E. Harris

R. Fairhurst

S. Aksoy

L. Slutsker

L. Kramer

A. Powers

N. Achee

D. Fidock

\section{5}

K. Kester

S. Volkman

E. Harris

P. Walker

S. Aksoy

L. Slutsker

L. Kramer

A. Powers

2014

K. Stuart

V. McGovern

D. Bausch

S. Volkman

E. Harris

P. Walker

S. Aksoy

L. Slutsker

\section{3}

P. Zimmerman

L. Ivers

K. Stuart

V. McGovern

D. Bausch

S. Volkman

E. Harris

P. Walker

\section{2}

S. Higgs

R. Steketee

A. LaBeaud

P. Zimmerman

K. Stuart

V. McGovern

D. Bausch

S. Volkman

2011

J. Breman

J. Hughes

S. Higgs

R. Steketee

D. Hill

P. Zimmerman

K. Stuart

V. McGovern

\section{0}

F. Richards

M. Wilson

J. Breman

J. Hughes

S. Higgs

R. Steketee

D. Hill

P. Zimmerman

\section{9}

R. Rabinovich

S. Weaver

F. Richards

M. Wilson

J. Breman

J. Hughes

S. Higgs

R. Steketee

\section{8}

J. Donelson

C. Sibley

R. Rabinovich

S. Weaver

F. Richards

M. Wilson

J. Breman

J. Hughes

\section{7}

G. Weil

A.C. White

J. Donelson

C. Sibley

R. Rabinovich

S. Weaver

F. Richards

M. Wilson

\section{6}

A. Magill

C. Panosian

G. Weil

A.C. White

J. Donelson

C. Sibley

R. Rabinovich

S. Weaver

\section{5}

A. James

M. Wilson

A. Magill

C. Panosian

G. Weil

A.C. White

J. Donelson

C. Sibley

\section{4}

L. Laughlin

J. Maguire

A. James

M. Wilson

A. Magill

C. Panosian

G. Weil

A.C. White

\section{3}

P. Hotez

A.A. James

L. Laughlin

A. Magill

J. Maguire

C. Panosian

C. Plowe

M.L. Wilson

2002

D.O. Freedman

P. Hotez

A.A. James

D.C. Kaslow

L. Laughlin

J. Maguire

C. Plowe

M.L. Wilson

\section{1}

D.O. Freedman

P. Hotez

D.C. Kaslow

L. Laughlin

J. Maguire

C. Plowe

R.E. Shope

T.E. Taylor

\section{0}

D.O. Freedman

M. Gottlieb

B.L. Herwaldt

P. Hotez

D.C. Kaslow

C. Plowe

R.E. Shope

T.E. Taylor

\section{9}

B.L. Doughty

D.O. Freedman

M. Gottlieb

B.L. Herwaldt

D.C. Kaslow

J.M. Ribeiro

R.E. Shope

T.E. Taylor

\section{8}

D.J. Gubler

J.C. Beier

B.L. Doughty

J.M. Ribeiro

M. Gottlieb

B.L. Herwaldt

R.E. Shope

T.E. Taylor
1997

D. McMahon-Pratt

B.M. Christensen

D.J. Gubler

J.C. Beier

B.L. Doughty

J.M. Ribeiro

M. Gottlieb

B.L. Herwaldt

\section{6}

E. Cupp

M.E. Wilson

D. McMahon-Pratt

B.M. Christensen

D.J. Gubler

J.C. Beier

B.L. Doughty

J.M. Ribeiro

\section{5}

M. Barry

H. Spencer

E. Cupp

M.E. Wilson

D. McMahon-Pratt

B.M. Christensen

D.J. Gubler

J.C. Beier

1994

C. Campbell

E. Jong

M. Barry

H. Spencer

E. Cupp

M.E. Wilson

D. McMahon-Pratt

B.M. Christensen

1993

S. Hoffman

R. Rico-Hesse

C. Campbell

E. Jong

M. Barry

H. Spencer

E. Cupp

M.E. Wilson

1992

D. Despommier

S. James

S. Hoffman

R. Rico-Hesse

C. Campbell

E. Jong 


\section{BOARD OF DIRECTORS (formerly known as Councilors), 1952-2019 (cont.)}

\author{
M. Barry \\ H. Spencer

\section{1} \\ R. Nussenzweig \\ J. Williams \\ D. Despommier \\ S. James \\ D. Krogstad \\ R. Rico-Hesse \\ C. Campbell \\ E. Jong
}

1990

G. Higashi

D. Wirth

R. Nussenzweig

J. Williams

D. Despommier

S. James

D. Krogstad

R. Rico-Hesse

1989

T. Frothingham

E. Ottesen

G. Higashi

D. Wirth

R. Nussenzweig

J. Williams

D. Despommier

S. James

\section{8}

D. Burke

R. Tesh

T. Frothingham

E. Ottesen

G. Higashi

D. Wirth

R. Nussenzweig

J. Williams

1987

R. Damian

M. Schultz

D. Burke

R. Tesh

T. Frothingham

E. Ottesen

G. Higashi

D. Wirth

\section{6}

G. Hillyer

W. Sodeman, Jr.

R. Damian

M. Schultz
D. Burke

R. Tesh

T. Frothingham

E. Ottesen

1985

J. Cook

D. Wyler

G. Hillyer

W. Sodeman, Jr.

R. Damian

M. Schultz

D. Burke

R. Tesh

\section{4}

J. Frenkel

D. Heyneman

J. Cook

D. Wyler

G. Hillyer

W. Sodeman, Jr.

R. Damian

M. Schultz

\section{3}

C. Diggs

T. Monath

J. Frenkel

D. Heyneman

J. Cook

D. Wyler

G. Hillyer

W. Sodeman, Jr.

1982

D. Colley

G. Craig, Jr.

C. Diggs

T. Monath

J. Frenkel

D. Heyneman

J. Cook

D. Wyler

1981

B. Cline

A. Senft

D. Colley

G. Craig, Jr.

C. Diggs

T. Monath

J. Frenkel

D. Heyneman

1980

P. Galindo

G. Wallace

B. Cline
A. Senft

D. Colley

G. Craig, Jr.

C. Diggs

T. Monath

1979

E. Barrett-Connor

M. Wolfe

P. Galindo

G. Wallace

B. Cline

R. Shope

D. Colley

G. Craig, Jr.

1978

T. Orihel

P. Russell

E. Barrett-Connor

M. Wolfe

P. Galindo

G. Wallace

B. Cline

R. Shope

1977

L. Ash

E. Chernin

T. Orithel

P. Russell

E. Barrett-Connor

M. Wolfe

P. Galindo

G. Wallace

\section{6}

H. Hoogstraal

K. Warren

L. Ash

E. Chemin

T. Orihel

P. Russell

E. Barrett-Connor M. Wolfe

\section{5}

R. Kuntz

L. Olivier

H. Hoogstraal

K. Warren

L. Ash

E. Chernin

T. Orihel

P. Russell

\section{4}

R. Shope

P. Weinstein
R. Kuntz

L. Olivier

H. Hoogstraal

K. Warren

L. Ash

E. Chemin

1973

A. Cheever

K. Johnson

R. Shope

P. Weinstein

R. Kuntz

L. Olivier

H. Hoogstraal

K. Warren

1972

A. Foster

L. Rosen

A. Cheever

K. Johnson

R. Shope

P. Weinstein

L. Olivier

R. Kuntz

1971

Q. Geiman

M. Voge

A. Foster

L. Rosen

A. Cheever

K. Johnson

R. Shope

P. Weinstein

1970

J. Steele

R. Jung

Q. Geiman

M. Voge

A. Foster

L. Rosen

A. Cheever

K. Johnson

1969

G. Otto

L. Schmidt

J. Steele

R. Jung

Q. Geiman

P. Thompson

A. Foster

L. Rosen

1968

L. Jacobs
D. Davis

G. Otto

L. Schmidt

J. Steele

R. Jung

Q. Geiman

T. Work

1967

E. Chernin

R. Lewert

L. Jacobs

M. Brooke

G. Otto

D. McMullen

J. Steele

R. Jung

1966

W. Downs

J. Oliver-González

W. McD. Hammon

J. Schwartzwelder

M. Brooke

L. Jacobs

D. McMullen

G. Otto

1965

C. Johnson

L. Stauber

W. Downs

J. Oliver-González

W. McD. Hammon

J. Schwartzwelder

M. Brooke

L. Jacobs

1964

L. Rozeboom

C. Philip

C. Johnson

L. Stauber

W. Downs

J. Oliver-González

W. McD. Hammon

J. Schwartzwelder

1963

A. Gabaldon

H. Most

L. Rozeboom

C. Phillip

C. Johnson

L. Stauber

W. Downs

J. Oliver-González 


\section{BOARD OF DIRECTORS (formerly known as Councilors), 1952-2019 (cont.)}

1962
F. Neva
J. Larsh, Jr.
A. Gabaldon
H. Most
L. Rozeboom
C. Phillip
C. Johnson
L. Stauber
1961
P. Russell
J. Smadel
M. Hertig
J. Larsh, Jr.
A. Gabaldon
H. Most
L. Rozeboom
C. Philip

1960

J. Henderson

J.A. Scott
P. Hussell
J. Smadel
M. Hertig
J. Larsh, Jr.
A. Gabaldon Venez
H. Most
1959
L. Stauber
W. Reeves
J. Henderson
J.A. Scott
P. Russell
J. Smadel
M. Hertig
J. Larsh, Jr.
1958
M. Balfour
T. Weller
P. Beaver

\section{W. Reeves}

J. Henderson

J.A. Scott

P. Russell

J. Smadel

1957

A. Sabin

J.J. Sapero

M. Balfour

T. Weller

P. Beaver

W. Reeves

J. Henderson

J.A. Scott

1956

C. Huff

S. Simmons

A. Sabin

J.J. Sapero

M. Balfour
T. Weller

P. Beaver

W. Reeves

1955

E. Lennette

G.R. Coatney

C. Huff

S. Simmons

A. Sabin

J.J. Sapero

M. Balfour

T. Weller

1954

T. Whayne

H. Johnstone

E. Lennette

G.R. Coatney

C. Huff

S. Simmons

A. Sabin
J.J. Sapero

1953

J.M. Andrews

D.L. Augustine

T.F. Whayne

H.G. Johnstone

E.H. Lennette

G.R. Coatney

C.G. Huff

S.W. Simmons

1952

G. Bradley

H. Most

J. Andrews

D. Augustine

T. Whayne

H. Johnstone

E. Lennette

G.R. Coatney

AMERICAN SOCIETY OF TROPICAL MEDICINE COUNCILORS, 1941-1951

\begin{abstract}
1951
J.C. Snyder

M. Hertig

G.R. Coatney

J.N. DeLamater

D.L. Augustine

G.M. Saunders

H.G. Johnstone

T.F. Whayne

1950

H.R. Cox

K.R. Lundeberg

J.C. Snyder

W.A. Sodeman

H. Most

J. DeLamater

D. Augustine

G. Saunders
\end{abstract}

1949

J. Andrews

M.E. Barnes
H.R. Cox

K.R. Lundeberg

W.A. Sodeman

J.C. Snyder

H. Most

J. DeLamater

1948

K.R. Lundeberg

H.R. Cox

W.A. Sodeman

H. Most

J. Andrews

P.E. Russell

M.E. Barnes

J.C. Snyder

1947

K.R. Lundeberg

H.R. Cox

J.F. Kessel

E.G. Hakansson

J. Andrews
O.R. McCoy

P.E. Russell

M.E. Barnes

1946

G.R. Callender

J.F. Kessel

E.G. Hakansson

J. Andrews

R.B. Watson

O.R. McCoy

P.E. Russell

M.E. Barnes

\section{5}

L.T. Coggeshall

A.J. Warren

G.R. Callender

R. Briggs Watson

J.F. Kessel

O.R. McCoy

E.G. Hakansson

E.I. Salisbury
1944

L.T. Coggeshall

T.J. LeBlanc

A.J. Warren

J.S. Simmons

G.R. Callender

R. Briggs Watson

J.F. Kessel

O.R. McCoy

1943

A.C. Reed

J.A. Curran

R.E. Dyer

T.J. LeBlanc

A.J. Warren

J.S. Simmons

G.P. Callender

R. Briggs Watson

1942

M.F. Boyd

L.T. Coggeshall
A.C. Reed

J.A. Curran

R.E. Dyer

T.J. LeBlanc

A.J. Warren

J.S. Simmons

1941

N. Paul Hudson

H.E. Meleney

M.F. Boyd

L.T. Coggeshall

A.C. Reed

J.A. Curan

R.E. Dyer

T.J. LeBlanc 


\section{MEDALS}

There are several medals given by the Society. The Walter Reed Medal, the Ben Kean Medal and the Joseph Augustin LePrince Medal are given sequentially every third year, whereas the Bailey K. Ashford Medal is given annually. The Donald Mackay Medal is given annually with recipients chosen in alternating years by the American Society of Tropical Medicine and Hygiene and the Royal Society of Tropical Medicine and Hygiene. These awards, as well as Certificates of Recognition, are selected by a committee composed of the immediate three past presidents of the Society. Nominations for these awards may be submitted by any member and should briefly summarize the candidate's qualifications and accomplishments. Young Investigator Awards are selected based on presentations made at the ASTMH Annual Meeting.

BAILEY K. ASHFORD MEDAL-

For distinguished work in tropical medicine. Initially the medal was to go only to workers under the age of 35 , but over time the specific upper age limit was gradually increased, and eventually dropped. The medal is awarded every year, and more than one award may be given.

Shannon Takala Harrison . . . . . . . . . . 2019

Joel Tarning . . . . . . . . . . . . . . . . 2019

Sharon Tennant. . . . . . . . . . . . . . . 2019

Wendy Prudhomme . . . . . . . . . . . . . 2018

Tyler Sharp. . . . . . . . . . . . . . . . . . . . . 2018

Margaret Kosek. . . . . . . . . . . . . . . . 2017

Stefan Kappe . . . . . . . . . . . . . . . . . . . . . 2016

Peter Crompton . . . . . . . . . . . . . . 2015

David A. Fidock . . . . . . . . . . . . . . . . . 2014

Slobodan Paessler. . . . . . . . . . . . . . . . . 2014

Elizabeth A. Winzeler . . . . . . . . . . . . . 2014

Rick M. Fairhurst. . . . . . . . . . . . . . . . . 2013

Simon I. Hay . . . . . . . . . . . . . . . . . . 2013

Kristy Murray. . . . . . . . . . . . . . . . . . . 2013

John Crump. . . . . . . . . . . . . . . . . . . . . 2012

Beth Kirkpatrick. . . . . . . . . . . . . . . . . 2012

Louise Ivers . . . . . . . . . . . . . . . . . . . . . 2011

Chandy John . . . . . . . . . . . . . . . . 2011

Joseph Vinetz . . . . . . . . . . . . . . . . . 2011

Eric Houpt . . . . . . . . . . . . . . . . . . . . 2010

Carolina Barillas-Mury . . . . . . . . . . . . 2010

Jesus Valenzuela . . . . . . . . . . . . . . . . . . 2009

Kevin Kain . . . . . . . . . . . . . . . . . . 2008

Michael Cappello . . . . . . . . . . . . . . . . 2007

Jeremy Farrar . . . . . . . . . . . . . . . . 2006

Thomas A. Wynn. . . . . . . . . . . . . . . 2006

Mary E. Wilson . . . . . . . . . . . . . . . 2005

Frank O. Richards . . . . . . . . . . . . . 2005

J. Kevin Baird . . . . . . . . . . . . . . . . 2005

Xinzhuan Su. . . . . . . . . . . . . . . . . . . 2004

Amy Klion. . . . . . . . . . . . . . . . . . . . . . . 2004

Peter Hotez . . . . . . . . . . . . . . . . . . 2003

Christopher Plowe . . . . . . . . . . . . . . 2002

Edward J. Pearce. . . . . . . . . . . . . . . 2002

Philip Scott. . . . . . . . . . . . . . . . . . . 2001

David Sacks . . . . . . . . . . . . . . . . . . . 2001

Barbara Mann . . . . . . . . . . . . . . . . . 2001

Terrie Taylor . . . . . . . . . . . . . . . . . . . . 2000

Thomas Nutman . . . . . . . . . . . . . . . . 2000

W. Ripley Ballou. . . . . . . . . . . . . . . . . . 2000

Jose Ribeiro . . . . . . . . . . . . . . . . . . . . . 1999

Barbara L. Herwaldt . . . . . . . . . . . . . 1999

Ayoade M. Oduola . . . . . . . . . . . . . . . . 1998

Donald A. Harn . . . . . . . . . . . . . . . . 1998

James W. Kazura . . . . . . . . . . . . . . . . . 1997

Stephanie James. . . . . . . . . . . . . . . . . . 1996

Dyann F. Wirth . . . . . . . . . . . . . . . 1995

Thomas E. Wellems . . . . . . . . . . . . . . 1995
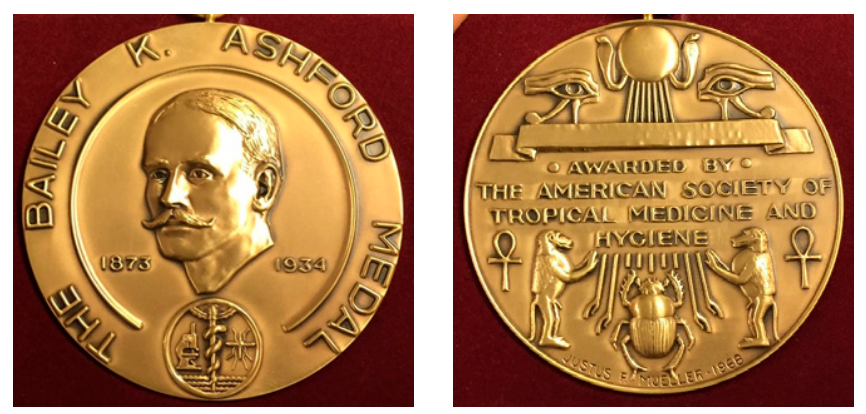

Jonathan I. Ravdin. . . . . . . . . . . . . . . 1994

Wilbur K. Milhous. . . . . . . . . . . . . . . 1993

Richard M. Locksley . . . . . . . . . . . . . 1993

Kamini Mendis . . . . . . . . . . . . . . . . . . 1993

Nancy G. Saravia. . . . . . . . . . . . . . . . 1993

Diane McMahon-Pratt . . . . . . . . . . . . . 1992

Charles H. Hoke, Jr. . . . . . . . . . . . . . . . 1992

Stephen L. Hoffman . . . . . . . . . . . . . 1992

Richard Yanagihara . . . . . . . . . . . . . . . . 1991

Peter F. Weller. . . . . . . . . . . . . . . . . . . 1991

Donald S. Burke. . . . . . . . . . . . . . . . . 1990

Alan F. Sher . . . . . . . . . . . . . . . . . 1989

Eric A. Ottesen . . . . . . . . . . . . . . . . . 1989

Daniel G. Colley. . . . . . . . . . . . . . . . . . . 1989

George V. Hillyer . . . . . . . . . . . . . . . 1986

Adel Mahmoud. . . . . . . . . . . . . . . . . . . 1983

Joel M. Dalrymple. . . . . . . . . . . . . . . 1983

Myron G. Schultz . . . . . . . . . . . . . . . 1980

James L. Hardy ....... . . . . . . . . . . 1977

Kenneth S. Warren . . . . . . . . . . . . . . 1974

Robert E. Shope . . . . . . . . . . . . . . . 1974

Karl M. Johnson . . . . . . . . . . . . . . 1971

Leon Rosen . . . . . . . . . . . . . . . . . . . 1968

Franklin A. Neva . . . . . . . . . . . . . . 1965

Eli Chernin . . . . . . . . . . . . . . . . 1961

Geoffrey M. Jeffery. . . . . . . . . . . . . . . . 1959

Paul P. Weinstein. . . . . . . . . . . . . . 1957

Joseph Greenberg. . . . . . . . . . . . . . 1954

Leo J. Jachowski, Jr. . . . . . . . . . . . . . . 1952

Robert J. Huebner. . . . . . . . . . . . . . . 1949

Jose Oliver-Gonzalez. . . . . . . . . . . . . . 1947

James Watt . . . . . . . . . . . . . . . . . . . 1945

Norman E. Topping . . . . . . . . . . . . . . . 1943

Lloyd E. Rozeboom . . . . . . . . . . . . . . 1941 
BEN KEAN MEDAL-

To recognize a clinician or educator whose dedication to clinical tropical medicine and impact on the training of students, fellows and/or practitioners of tropical medicine is in keeping with the tradition established by Dr. Kean.

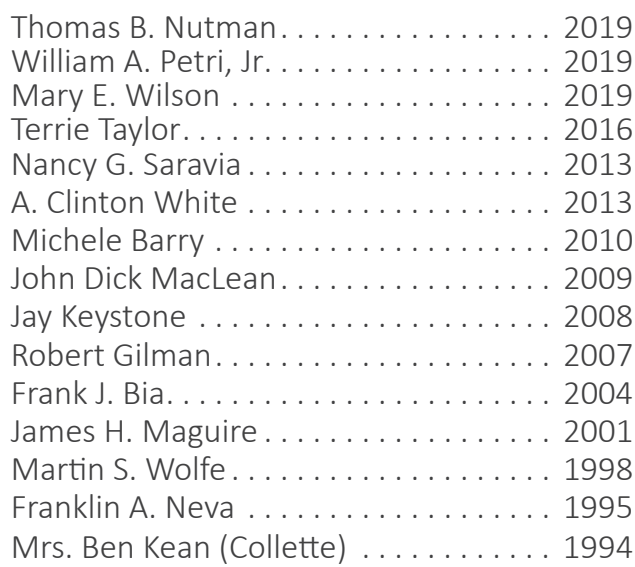
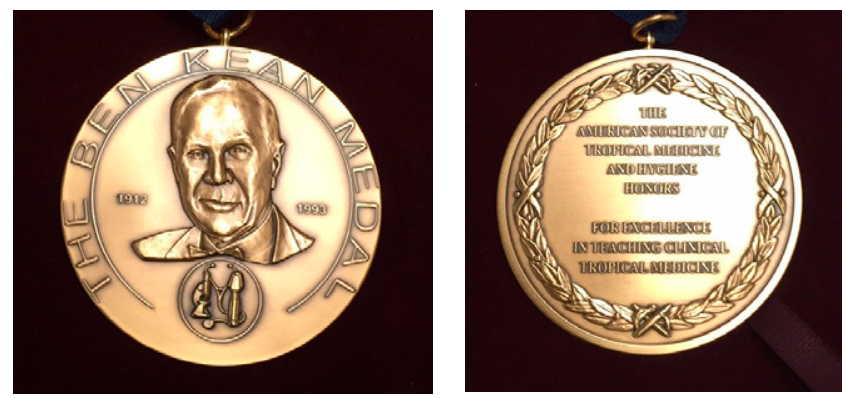

JOSEPH AUGUSTIN LePRINCE MEDAL-

In recognition of outstanding work in the field of malariology.

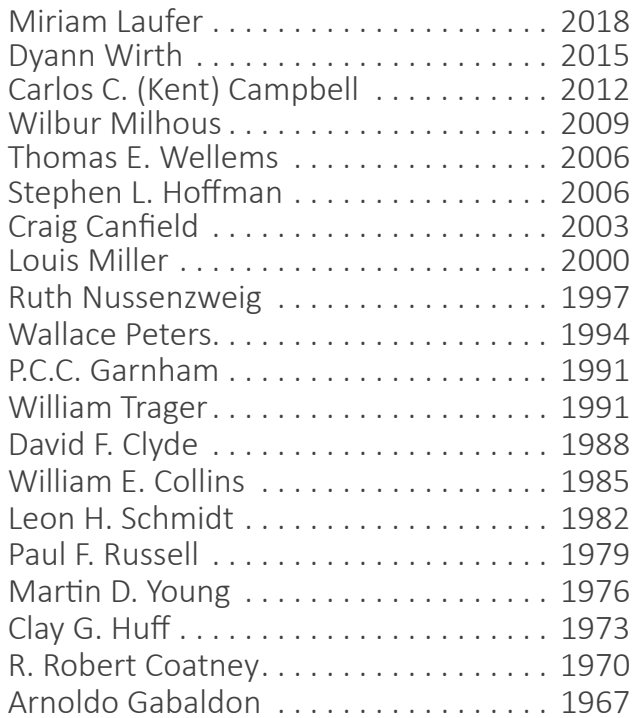
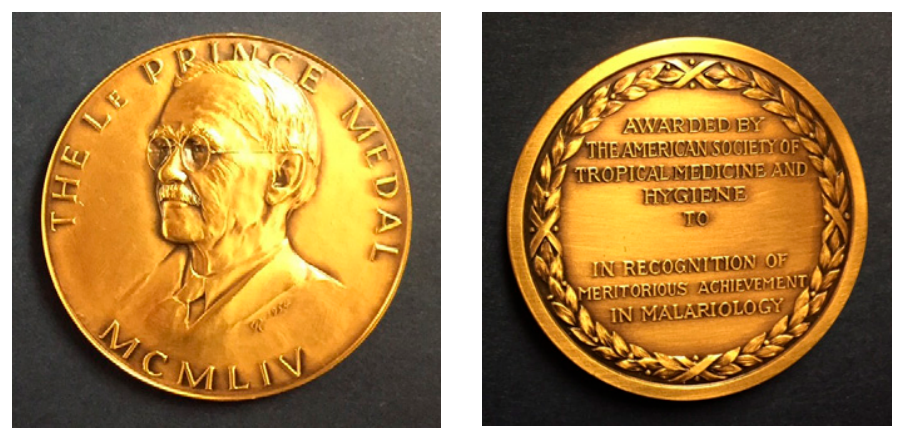

Don E. Eyles. . . . . . . . . . . . . . 1964

Justin Andrews ... . . . . . . . . . . . 1960

Louis L. Williams, Jr. . . . . . . . . . 1957

Brian Maegraith . . . . . . . . . . . . . 1954

Joseph Augustin LePrince .......... 1951

\section{LUDLOW MEDAL -}

This medal is the first to be named after a female icon in tropical medicine. It recognizes honorees for their inspirational and pioneering spirit, whose work represents success despite obstacles and advances the field of tropical medicine. Clara Southmayd Ludlow (1852-1924) was an entomologist with scientific zeal and tenacity who battled the odds of age, gender and skepticism of women in the sciences to advance the understanding of tropical medicine.
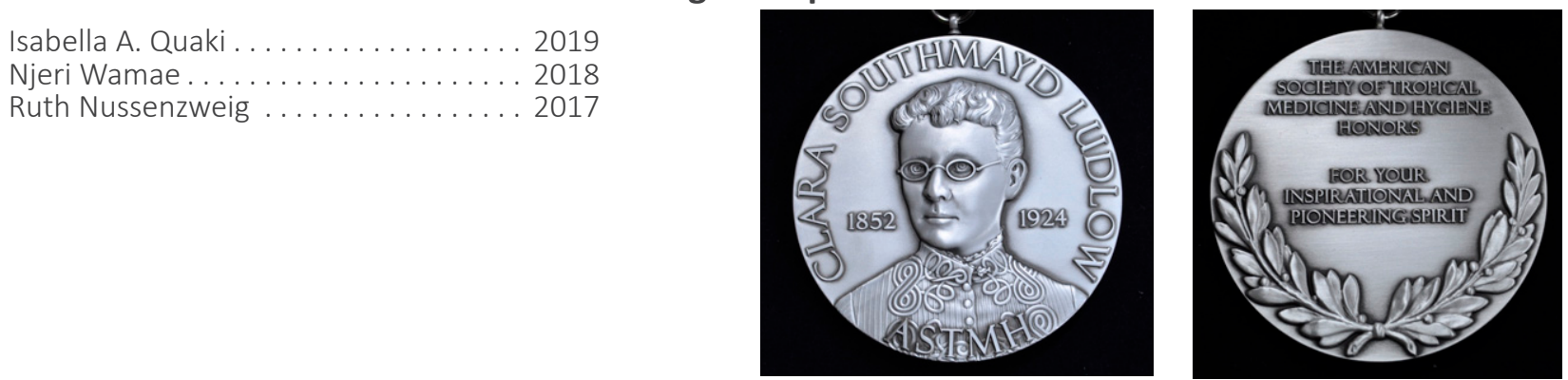
DONALD MACKAY MEDAL-

For outstanding work in tropical health, especially relating to improvements in the health of rural or urban workers in the tropics. Preference is given to suitable medically qualified individuals. The Medal is awarded annually with the Royal Society of Tropical Medicine and Hygiene selecting awardees in even- numbered years and the American Society of Tropical Medicine and Hygiene selecting recipients in odd-numbered years.
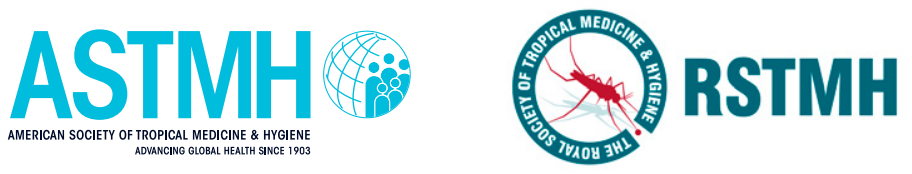

\begin{tabular}{|c|c|}
\hline Christopher King & 2019 \\
\hline Ahmed Hassan Fahal. & 2018 \\
\hline Patrick Lammie. . & 2017 \\
\hline Moses Bockarie. & 2016 \\
\hline G. Dennis Shanks & 201 \\
\hline Alimuddin Zumla. & 2014 \\
\hline Myron M. Levine. & 2013 \\
\hline Gary J. Weil. & 2013 \\
\hline Tewolde Gebremeskel. . & 20 \\
\hline David Sack & 2011 \\
\hline R. Bradley Sack & 2011 \\
\hline Tran Tinh Hien . & 20 \\
\hline Jane Cardosa. & 20 \\
\hline nthony Bryceson. & 200 \\
\hline David Molyneux & 200 \\
\hline Paul E.M. Fine. . & 2006 \\
\hline David Heymann & 20 \\
\hline Alan Fenwick. & 200 \\
\hline Eric Ottesen. & 20 \\
\hline Joseph Cook & 20 \\
\hline L. Tulloch. . & 2 \\
\hline anklin Neva & \\
\hline H & \\
\hline Groot & \\
\hline
\end{tabular}
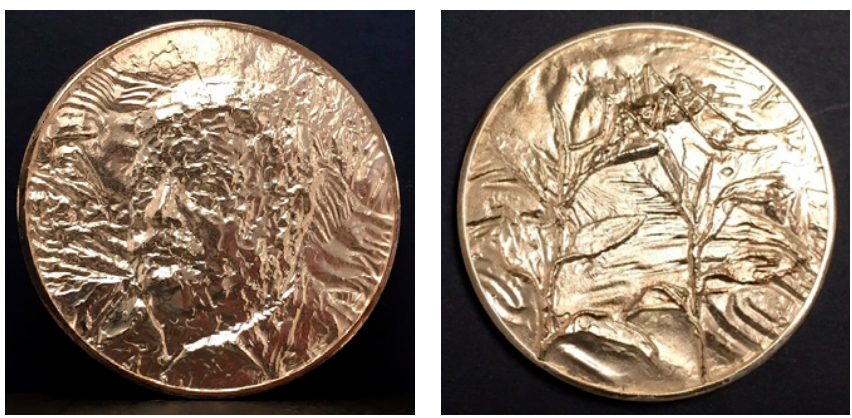

Ahmed El Hassan . . . . . . . . . . . 1996

Alfred A. Buck... . . . . . . . . . . . . 1995

Jill Seaman .................. 1994

Warren L. and Gretchen Berggren ... . 1993

Bernard Koucher. . . . . . . . . . . . . . . . 1992

Brian M. Greenwood . . . . . . . . . . . . . 1991

Ralph H. Henderson . . . . . . . . . . . . . . 1990

\section{WALTER REED MEDAL-}

To recognize distinguished accomplishment in the field of tropical medicine.

Scott Halstead . . . . . . . . . . . . 2017

Alan J. Magill (in Memoriam). . . . . . . . 2015

Scott Weaver. . . . . . . . . . . . . . . . . 2014

Louis Miller . . . . . . . . . . . . . . . . . . . . . 2011

Richard L. Guerrant. . . . . . . . . . 2008

Karl M. Johnson . . . . . . . . . . . . . . . . 2005

Thomas Monath . . . . . . . . . . . . . . . . . . 2002

Philip K. Russell . . . . . . . . . . . . . . . . . 1999

Thomas H. Weller . . . . . . . . . . . . . . . 1996

Robert E. Shope . . . . . . . . . . . . . . . 1993

George B. Craig, Jr. . . . . . . . . . . . . . . 1993

Donald A. Henderson . . . . . . . . . . . . 1993

Willy Burgdorfer. . . . . . . . . . . . . . . . 1990

Paul C. Beaver . . . . . . . . . . . . . . . . . 1990

William C. Reeves . . . . . . . . . . . . . . . 1987

Wilbur G. Downs. . . . . . . . . . . . . . . . . 1984

Harry Most . . . . . . . . . . . . . . . . . . . 1981

Harry Hoogstraal. . . . . . . . . . . . . . . . . 1978

Robert Briggs Watson. . . . . . . . . . . . . 1975

Fred L. Soper . . . . . . . . . . . . . . . . . . . 1972

Albert B. Sabin . . . . . . . . . . . . . . . . . 1969

Henry E. Meleney. . . . . . . . . . . . . . . . 1966

Ernest Carroll Faust . . . . . . . . . . . . . . 1963

William W. Cort. . . . . . . . . . . . . . . . . 1962

Sir Gordon Covell . . . . . . . . . . . . . . . . 1960
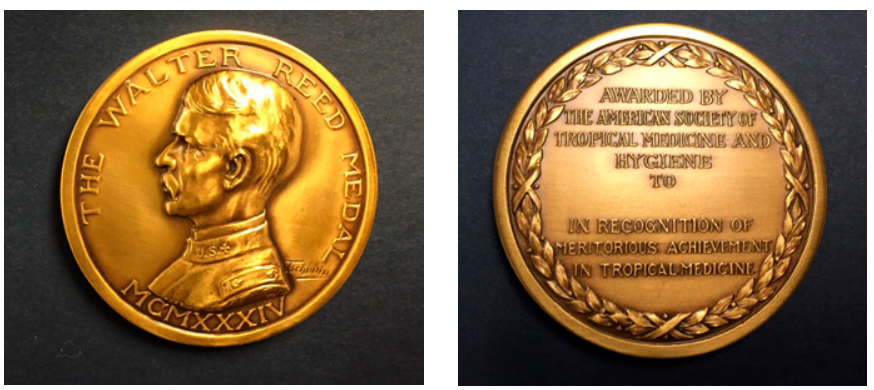

Willard H. Wright . . . . . . . . . . . . . 1958

Karl F. Meyer . . . . . . . . . . . . . . . . . . 1956

Lewis W. Hackett. . . . . . . . . . . . . . . . 1953

Rolla E. Dyer . . . . . . . . . . . . . . . . . . 1950

N. Swellengrebel. . . . . . . . . . . . . . . . . 1948

Paul F. Russell . . . . . . . . . . . . . . . . . . 1946

James S. Simmons. . . . . . . . . . . . . . . . 1944

Gustavo Campenema . . . . . . . . . . . . . . 1942

Carlos J. Finlay. . . . . . . . . . . . . . . . . . . 1942

Herbert C. Clark . . . . . . . . . . . . . . . 1940

William B. Castle . . . . . . . . . . . . . . . . 1939

The Rockefeller Foundation . . . . . . . . 1936

Mrs. Walter Reed . . . . . . . . . . . . . 1936 
COMMUNICATIONS AWARD-This award recognizes content that enhances the public's understanding and appreciation of tropical medicine research, clinical practice and/or policy.

2019

Martin Enserink

Science

"On a remote Pacific island, this doctor has revived a 60-year quest to eradicate a disfiguring disease"

\section{8}

Lena H. Sun

The Washington Post

"Chasing a Killer"

\section{7}

William Brangham, Jon Cohen and Jason Kane

PBS Newshour

"Why a Kenyan Island May Teach the World How to Beat AIDS"

\section{6}

Warren Cornwall

Science

"A Plague of Rats"

\section{5}

Kevin Sack, Sheri Fink, Pam Belluck, Adam Nossiter and Celia Dugger (Editor) The New York Times

"How Ebola Roared Back"
2014

Leslie Roberts

Science

"The Art of Eradicating Polio"

2013

Shelly Xie

University of Texas

Southwestern Medical Center

2012

Jason Gate and Adi Narayan

Bloomberg News

"The Scourge of the Superbugs"

2011

The Financial Times

Science Magazine

2010

Tina Rosenberg*

National Geographic

"The Burden of Thirst"

2009

Vivienne Walt

Time Magazine

"Death in Birth"

\section{8}

Charles Piller and Doug Smith Los Angeles Times

"Unintended Victims of Gates Foundation Generosity"

2007

Michael Leahy

The Washington Post

"Breaking the Cycle"

\section{6}

Celia Dugger and Donald McNeil, Jr. The New York Times**

"On the Brink"

\section{5}

Leslie Roberts

Science Magazine

"Rotavirus Vaccines' Second Chance"

* donated to WaterAid America, Inc.

** cash prize declined per NYT policy

\section{Fellowships/Awards/Annual Meetings/Recognitions}

\section{FELLOW OF ASTMH \\ (FASTMH)}

Class of 2019

Carter Diggs

Albert Icksang Ko

Amy Klion

Daniel Leung

Siddhartha Mahanty

Julie Pavlin

Anne Rimoin

Mary Wilson

\section{Class of 2018 \\ James Diaz \\ Eva Harris \\ Charles King \\ N. Regina Rabinovich}

\section{Class of 2017}

John H. Adams

Carolina V. Barillas-Mury

Robert Frederic Breiman

Robert Edelman

David O. Freedman

Mark G. Kortepeter

Gregory J. Martin

Steven R. Meshnick

Danny Arnold Milner

Hira L. Nakhasi

Daouda Ndiaye
B. Kim Lee Sim

Christopher W. Woods

\section{Class of 2016}

Elizabeth Barnett

Stephen M. Beverley

Carol D. Blair

Donald S. Burke

Martin Cetron

Barnett L. Cline

Daniel G. Colley

Joseph A. Cook

John R. David

David Fidock

Duane Gubler

Richard L. Guerrant

Scott B. Halstead

Stephanie James

Karl M. Johnson

Irving G. Kagan

Peter J. Krause

Carole A. Long

Claire B. Panosian

Frank O. Richards

Philip Russell

G. Dennis Shanks

Donald S. Shepard

Mary M. Stevenson

Nikos Vasilakis

Karl A. Western

A. Clinton White
Dyann Wirth

Class of 2015

J. Kevin Baird

Frank Bia

Hector Gorbea

Daniel Gordon

Christopher King

Beth Kirkpatrick

William Klein

Nirbhay Kumar

Sanjai Kumar

Jean Lang

David Morens

Amy Morrison

Nicanor Obaldia

Sheral Patel

Mark Polhemus

John Sanders

Lynn Soong

J. Brice Weinberg

Class of 2014

Daniel G. Bausch

Nora Besansky

A.D. Brandling-Bennett

Paul J. Brindley

Lin Chen

Jan E. Conn

James F. Cummings

Rick M. Fairhurst
Robert A. Gasser

Davidson H. Hamer

Simon I. Hay

Eric R. Houpt

Chandy C. John

S. Patrick Kachur Louis V. Kirchhoff

Laura D. Kramer

Shirley Luckhart

Peter C. Melby

Bernard Nahlen

Latha Rajan

Philip Rosenthal

Thomas W. Scott

Alan M. Spira

Stephen J. Thomas

Thomas R. Unnasch

David H. Walker

Scott C. Weaver

Class of 2013

Barry J. Beaty

Maria Elena Bottazzi

Stephen B. Calderwood

Philip E. Coyne

Robert H. Gilman

Lars Hviid

M. Patricia Joyce

Niranjan Kanesa-Thasan

Karen Kotloff

Dennis E. Kyle 
Anne McCarthy

J. Glenn Morris

Eric A. Ottesen

Christopher Plowe

Laurence Slutsker

Oyewale Tomori

Patricia F. Walker

Douglas Walsh

Martin S. Wolfe

\section{Class of 2012}

Serap Aksoy

Carlos C. Campbell

Bruce M. Christensen

John A. Crump

D. Gray Heppner

Stephen L. Hoffman

Duane R. Hospenthal

Noreen A. Hynes

Anthony A. James

Elaine C. Jong

Kent E. Kester

Walter (Ted) F. Kuhn

Susan McLellan

Thomas B. Nutman

Sarah K. Volkman

Gary J. Weil

\section{Class of 2011}

Michele Barry

Josh D. Berman

Joel G. Breman

Stephen Higgs

David R. Hill

George V. Hillyer

Peter J. Hotez

James M. Hughes

James W. Kazura

Donald J. Krogstad

Myron M. Levine

Alan J. Magill

Victoria McGovern

Wilbur K. Milhous

Louis H. Miller

Thomas P. Monath

William A. Petri, Jr.

Ann M. Powers

Edward T. Ryan

William Stauffer

Richard W. Steketee

Joseph M. Vinetz

Thomas E. Wellems

Peter F. Weller

Mary Elizabeth Wilson

\section{Honorary International} Fellow of ASTMH

(formerly known as Honorary Member)

\section{9}

Ahmed, Tahmeed

Bangladesh

Grobusch, Martin

Netherlands

\author{
Menendez, Clara \\ Spain \\ Ringwald, Pascal \\ Switzerland \\ Saul, Allen \\ Italy \\ 2018 \\ Anstey, Nicholas \\ Australia \\ Bhutta, Zulfiqar \\ Canada \\ da Costa Vasconcelos, Pedro \\ Brazil \\ McGready, Rose \\ Thailand \\ ter Kuile, Feiko \\ United Kingdom
}

2017

Kremsner, Peter

Germany

McCarthy, James

Australia

Muyembe, Jean Jacques

Dem. Rep. of Congo

Shaw, Jeffrey

Brazil

Sundar, Shyam

India

2016

Brown, Graham

Australia

Correa-Oliveira, Rodrigo

Brazil

Doumbo, Ogobara

Mali

Haque, Rashidul

Bangladesh

Oduola, Ayoade

Nigeria

2015

Aaskov, John

Australia

Binka, Fred

Ghana

Flisser, Ana

Mexico

Kalil, Jorge

Brazil

Leke, Rose Gana Fomban

Cameroon

2014

Enria, Delia

Argentina

Ito, Akira

Japan
Molyneux, David

United Kingdom

Reis, Mitermayer

Brazil

Sow, Samba

Mali

2013

Alonso, Pedro

Spain

Carvalho, Edgar

Brazil

Nosten, Francois

Thailand

Pinheiro, Francisco

Brazil

Qadri, Firdausi

Bangladesh

2012

Fenwick, Alan

United Kingdom

Lima, Aldo

Brazil

Marsh, Kevin

Kenya

Tanner, Marcel

Switzerland

2011

Hien, Tran Tinh

Vietnam

Jeronimo, Selma

Brazil

2010

McManus, Donald

Australia

Pasvol, Geoffrey

United Kingdom

2009

Greenwood, Brian

United Kingdom

Lainson, Ralph

Brazil

2008

Ambroise-Thomas, Pierre

France

Sousa, Anastacio

Brazil

2007

Sinden, Robert

United Kingdom

Swanepoel, Robert

South Africa

2006

Good, Michael

Australia
Horton, John

United Kingdom

2005

Killick-Kendrick, Robert

France

White, Nicholas

Thailand

2004

Rieckmann, Karl

Australia

Seth, Pradeep

India

2003

Duke, Brian

United Kingdom

Warrell, David

United Kingdom

2002

Gotuzzo, Eduardo

Peru

2000

Nisalak, Ananda

Thailand

Portaels, Francoise

Belgium

1999

Bryceson, Anthony Australia

1998

Prata, Aluizio

Brazil

1997

Feachem, Richard

United States

Homeida, Mamoun

Sudan

1996

Degremont, Antoine

Laos

Gazzinelli, Giovanni Brazil

1995

Peters, Wallace

United Kingdom

1994

Capron, Andre

France

Molineaux, Louis

Switzerland 


\section{Fellowships/Awards/Annual Meetings/Recognitions}

\section{3}

Alpers, Michael

Australia

Lee, Ho Wang

South Korea

1991

De Araujo Andrade, Zilton

Brazil

Godal, Tore

Switzerland

Mata, Leonardo

Costa Rica

1990

Pawlowski, Zbigniew

Poland

1988

Gilles, Herbert

United Kingdom

1987

Garcia, Edito G.

Philippines

Lucas, Adetokunbo

Nigeria

1986

Janssens, Pieter G.

Belgium

1984

Soh, Chin

South Korea

1982

Convit, Jacinto

Venezuela

Zhong, Hui-Lan

Peoples Republic of China

1981

Groot, Hernando

Colombia

1980

Zagreb, Jelka Vesenjak

Croatia

1979

Harinasuta, Chamlong

Thailand

1971

Sasa, Manabu

Japan

1963

Hsieh, Hsien-Chen

Taiwan R.O.C.
Lifetime Members Western, Karl

Aultman, Kathryn

Bhattarai, Achuyt

Blower, Donald

Boulware, David

Box-Hutchinson, Louise

Brennen, Roy

Brink-Legters, Linda

Burkot, Thomas

Cahill, John

Chapman, Louisa

Chhoeu, Austin

Colley, Daniel

Cummings, James

Dohn, Michael

Dooley, John

Dover, Arthur

Farooq, Ahmed

Fischer, Stephen

Graninger, Wolfgang

Guerrant, Richard

Hadley, Lesca

Hay, Simon

Herrick, Timothy

Hoadley, Deborah

Houpt, Eric

Hussein, Ahmed

Kaneko, Osamu

Kapella, Bryan

Kazura, James

Khalil, Hamed

Ko, Albert

Krogstad, Donald

LeDuc, James

Lin, Andrew

Macdonald, Etta Mae

Maguire, James

Malin, Howard

Markus, Miles

Massey, Joel

Murphy, James

Panosian, Claire

Petri, William

Plowe, Chris

Price, Todd

Rajan, Latha

Roberts, Bertram

Rudnick, Albert

Ryan, Edward

Sagebiel-Maley, Stephanie

Saint-Yves, Ian

Seefelder, Christian

Seitz, H.M.

Smith, Darvin

Sollers-Riedel, Helen

Spira, Alan

Stoler, Justin

Tchuem Tchuente, Louis-Albert

Tolia, Niraj

Valencia, Cynthia

Vierheilig, Jacqueline

Vogel, Carl-Wilhelm

Wellems, Thomas

Weller, Peter

Wolfe, Nathan

Zyzak, Michae

Alvarez, Julio

Arata, Andrew

Ayalde, Jaime

Bacchi, Cyrus

Bailey, Virginia

Baker, Walter

Bart, Kenneth

Berry, Richard

Berzins, Klavs

Bhatt, Pravin

Botero, David

Brosius, Otto

Brown, Arthur

Brown, Joel

Bryan, Ralph

Burga, Roger

Burry, Mary

Cain, George

Chen, Eng-Rin

Chin, Tom

Chin, William

Clark, Sandra

Cline, Barnett

Cook, Joseph

Craig, John

Crane, George

Cupp, Ed

David, John
White, A. Clinton

Whitty, Christopher Donelson, John

\section{Emeritus Members}

Acholonu, Alex

Allegra, Donald

Anderson, Clyde

Arbuthnot, James

Armstrong, Joseph

Ash, Lawrence

Barrett-Connor, Elizabeth

Bergquist, Robert

Biddle, Marjori

Bondi, Amedeo

Bosworth, Anthony

Boudreau, Ellen

Brooke, Marion

Brown, Kenneth

Brubaker, Merlin

Burchard, Gerd

Calisher, Charles

Campbell, William

Castro-Estrada, Santiago

Chapman, William

Chinel, Luis Valve

Christensen, Bruce

Clark, Lawrence

Cuckler, Ashton

Darsie, Richard

Davison, Thomas

Denlinger, David

Diamond, Louis

Doenhoff, Michael
Eckerlin, Ralph

Edelman, Robert

Eldridge, Bruce

Elisberg, Bennett

Ennis, Francis

Erickson, Duane

Estrin, William

Feldman, Roger

Felsenfeld, Ambhan

Ferris, Deam

Fleckenstein, Lawrence

French, George

Frierson, J. Gordon

Frothingham, Thomas

Genther, Clara Sesler

Gerberg, Eugene

Ghosh, Tara

Gill, Jag

Gleich, Gerald

Goldsmid, J.M.

Goldsmith, Robert

Gordon, Martin

Gottlieb, Michael

Graf, Penelope

Gubler, Duane

Gundelfinger, Benjamin

Haas, Victor

Hale, De Von

Harper, Kathleen

Hayes, Curtis

Herman, Robert

Heyneman, Donald

Hibbs, Richard

Himonas, Chris

Hoke, Charles

Holdenried, Robert

Hopps, Howard

Horton, John

Hougesen, Bent

$\mathrm{Hu}$, Xiao-Su

Hughes, James

Hussey, Kathleen

Hyland, Kerwin

Imperato, Pascal

Iversson, Lygia

Jaureguiberry, Ginette

John, David

Johnson, Karl

Johnson, Phyllis

Jong, Elaine

Jordan, William

Jung, Andre

Justines, Gustavo

Kagan, Irving

Kager, Piet

Kalter, S.S.

Katz, Frank

Kawashima, Kenjiro

Kawata, Kazuyoshi

Kern, Peter

Klebanoff, Seymour

Kliks, Michael 
Knopf, Paul

Kreutzer, Richard

Kuhn, Sharon

Kulcsar, David

Kutz, Frederick

Kwan, Clare

Lane, Robert

Lange, James

Lassmann, G. W.

Lawrence, Dale

Lelijveld, Jan

Lennox, Robert

Levin, Norman

Levine, Norman

Lewis, George

Ley, Herbert

Londner, Mauricio

Mahdy, M.S.

Malhotra, Indu

Mangiafico, Joseph

Mansour, Noshy

Mantel, Rita

Marcolongo, Assunta

Marcus, Leonard

McCall, John

McMahon-Pratt, Diane

McMillan, Kenneth

McMorrow, Clyde

McNeeley, David

McQuay, Russell

Mertz, Gregory

Meuwissen, Joseph

Meyer, Ernest

Meyers, Wayne

Miller, Albert

Miller, Larry
Murphy, Frederick

Nalin, David

Newson, Harold

Nisbett, Richard

Nothdurft, Hans

Nowell, Wesley

Ogden, Louis

Orbach, Hyman

Palmer, T. T.

Pankey, George

Patterson, Thomas

Pearson, Richard

Person, Donald

Pien, Francis

Podgore, John

Powell, Robin

Punjabi, Narain

Quarterman, Kenneth

Rees, William

Reisen, William

Reller, Lyman

Remington, Jack

Ribeiro, F.M.

Rice, Justus

Roberts, R H

Rosato, Robert

Rossan, Richard

Rowley, Wayne

Ruchman, Isaac

Russell, Philip

Salafsky, Bernard

Sanchez, Temistocles

Sapico, Francisco

Schantz, Peter

Schapiro, Mark

Schlesinger, Jacob
Schmutzhard, Erich

Schwabe, Calvin

Scott, Robert

Sexton, John

Shaw, Robert

Sherman, Irwin

Shiff, Clive

Shrager, Joel

Sibley, Carol

Smith, James

Smith, Joseph

Sodeman, William

Sogandares, Franklin

Sonenshine, Daniel

Sorensen, Kurt

Steel, Cathy

Sulahian, Annie

Sulzer, Alexander

Swartzwelder, J. Clyde

Targett, Geoffrey

Tesh, Robert

Tikasingh, Elisha

Tonn, Robert

Top, Franklin

Trpis, Milan

Tully, Joseph

Turell, Michael

Tzianabos, Theodore

Underman, Arvid

Van der Esch, Eric

Vanden Bossche, Hugo

Vanderberg, Jerome

Vodkin, Michael

von Sonnenburg, Frank

Walters, Laurel

Walton, Thomas
Walzer, Peter

Webster, H. Kyle

Weisfeld, Jason

Weiss, Margaret

Weiss, Niggi

Welborn, Charles

Weller, Richard

Wendt, Rosamund

Wery, Marc

Wikel, Stephen

Willcox, Michael

Wilson, Craig

Winter, Phillip

Wittner, Murray

Wykoff, Dale

Young, Frank

Young, Frank

Yuill, Thomas

Yunker, Conrad

Zeledon, Rodrigo

\section{AFFILIATE MEMBERS}

Affiliate membership is an opportunity for a company, corporation, foundation or other type of organization to support and participate in the society and its mission.

Donor

Peter Melby

University of Texas Medical Branch

\section{FELLOWSHIPS}

\section{ALAN J. MAGILL FELLOWSHIP - \\ This fellowship was created by the ASTMH then Council, now Board, in partnership with the Bill \& Melinda Gates Foundation to honor the life, example and legacy of Alan J. Magill, MD, FASTMH, a widely recognized and respected leader in the global tropical medicine community.}

2019

Deusdedith Ishengoma

National Institute for Medical Research (NIMR), Tanga

Medical Research Centre, Tanzania

2018

Abhilasha Karkey

Patan Hospital, Nepal
2017

Pedro Aide

Centro de Investigação de Saúde de Manhiça,

Mozambique 


\section{BURROUGHS WELLCOME FUND-ASTMH POSTDOCTORAL FELLOWSHIP IN TROPICAL INFECTIOUS DISEASES- This award provides research support for individuals enrolled in infectious disease fellowship programs who will spend a portion of their time working on research in a tropical or developing area.}

$\mathbf{2 0 1 9}$
Emily Ciccone
University of North Carolina School of
Medicine
Rose Lee
Boston Children's Hospital, Beth Israel
Deaconess Medical Center
Kevin Steiner
University of Virginia
$\mathbf{2 0 1 8}$
Lisa Bebell
University of Maryland
DeAnna Friedman-Klabanoff
Massachusetts General Hospital
Peyton Wilson
University of North Carolina, Chapel Hill

2017

Tara Bouton

Brown University

Patrick Cudahy

Yale University

Matthew Ippolito

Johns Hopkins University School of

Medicine

2016

Sarah-Blythe Ballard

Johns Hopkins Bloomberg School of

Public Health

Ruvandhi Nathavitharana

Beth Israel Deaconess Medical Center/

Harvard Medical School

Jonathan Parr

University of North Carolina at

Chapel Hill

\section{5}

Lauren Cohee

University of Maryland

Heidi Hillesland

University of Washington

Matthew Kelly

Duke University Medical Center

2014

Katherine Dobbs

Case Western Reserve University

John Openshaw

Stanford University
James Platts-Mills

University of Virginia

2013

Soumya Chatterjee

National Institute of Allergy and

Infectious Diseases

Andrew DiNardo

Baylor College of Medicine

Emily Wong

Massachusetts General Hospital

2012

Jason Andrews

Massachusetts General Hospital

Natalie Bowman

University of North Carolina

Claudia Denkinger

McGill University

2011

Kathryn Dupnik

Weill Cornell Medical College

Michelle Hsiang

University of California at

San Francisco

Jessica Lin

University of North Carolina

2010

Prasanna Jagannathan

University of California San Francisco

Daniel Leung

Massachusetts General Hospital

2009

Karen Jacobson

Massachusetts General Hospital

Lynn Matthews

Beth Israel Deaconess Medical

Center

Mark Travassos

University of Maryland

\section{8}

Nina Lin

Massachusetts General Hospital

Scott Peterson

Harvard Medical School

Joseph Tucker

Massachusetts General Hospital
2007

Scott Heysell

Yale University

Matthew Laurens

University of Maryland

Amber Read

University of New Mexico Health

Sciences Center

2006

Ryan Carroll

Northwestern University

Risa Hoffman

University of California at Los Angeles

Rebecca Plank

Brigham and Women's Hospital

2005

David Christiansen

University of Utah

\section{4}

Iracema Arevalo

New York University

Paul Lantos

Children's Hospital Boston

Danny Milner

Brigham and Women's Hospital

2003

Anusha Gunasekera

Harvard School of Public Health

Megan Reller

Children's Hospital Boston

2002

Ajay R. Bharti

University of California at San Diego

Holly Murphy

Tulane University

\section{1}

Aric Gregson

University of Maryland School of Medicine

Regina LaRoque

Massachusetts General Hospital

Krystn R. Wagner

Johns Hopkins University 


\section{CENTENNIAL TRAVEL AWARD IN BASIC SCIENCE TROPICAL DISEASE RESEARCH-}

This award provides support for a short-term research experience in the tropics to pursue studies in molecular, cellular or immunological aspects of tropical infectious diseases.

\section{9}

Camila Coelho

National Institutes of Health, National Institute of Allergy and Infectious

Diseases

\section{8}

Katherine Siddle

Broad Institute of the Massachusetts Institute of Technology (MIT) and

Harvard University

\section{7}

Melissa Conrad

University of California, San Francisco

Usheer Kanjee

Harvard T.H. Chan School of Public

Health

\section{6}

Heather Glasgow

Albert Einstein College of Medicine

\section{5}

Brie Falkard

Massachusetts General Hospital

\section{4}

Anna Babakhanyan

University of Hawaii at Manoa

\section{3}

Sarah-Blythe Ballard

Johns Hopkins University
2012

Amy Bei

Harvard School of Public Health

2011

Anne Frosch

University of Minnesota

2010

Amma Semenya

Centers for Disease Control and Prevention

2009

Robert Comer

Wake Forest University Baptist Medical Center

2008

Jessica Lin

University of North Carolina, Chapel Hill

2007

Judith Denery

Scripps Research Institute

Amber Read

University of New Mexico

2006

Erika Lamb

Uniformed Services University of the

Health Sciences
Stephen Popper

Stanford University

Scott Westenberger

Scripps Research Institute

\section{5}

Annette Kraus

University of North Carolina

2004

Gnanasekar Munirathinam

University of Illinois College of Medicine

Kristine Peterson

University of Virginia

Lisa Steele

Centers for Disease Control and

Prevention

Rhoel Dinglasan

Johns Hopkins School of Public Health

Sanjay Mehta

University of California at San Diego

2003

Katie Kurkjian

Centers for Disease Control and

Prevention

2002

Myaing Myaing Nyunt Johns Hopkins University

\section{ROBERT E. SHOPE INTERNATIONAL FELLOWSHIP IN INFECTIOUS DISEASES-}

This award provides support for a short-term research experience in the tropics in the area of arbovirology and/or emerging diseases.

2019

Anna Fagre

Colorado State University

\section{8}

Cameron Myhrvold

Broad Institute of the Massachusetts Institute of Technology (MIT) and Harvard University

\section{7}

Amy Krystosik

Stanford University School of Medicine

\section{6}

Kayla Barnes

Broad Institute of MIT and Harvard University

Steven Hatch

University of Massachusetts Medical School
2015

Claire Heath

Stanford University

2013

Jesse Waggoner

Stanford University

Sansanee Noisakran

Emory University

2012

Gillian Eastwood

New York State Department of Health

2011

Jonathan Auguste

The University of the West Indies
2010

Sarah Billeter

Centers for Disease Control and

Prevention

2009

Andrew Haddow

University of Texas Medical Branch

\section{8}

A. Desiree LaBeaud

Case Western Reserve University

\section{7}

Rebekah Kent

Centers for Disease Control and Prevention

\section{6}

Christine Hice

University of New Mexico 


\section{BENJAMIN H. KEAN TRAVEL FELLOWSHIP IN TROPICAL MEDICINE- This fellowship is designed to support medical students, house staff and fellows involved in clinical or research electives in tropical areas.}

2019

Eve Ameen

Stony Brook School of Medicine

Danielle Amundsen

Johns Hopkins School of Medicine

Tiffany Borbon

University of lowa Carver College of Medicine

Bickey Chang

University of Virginia School of Medicine

Hannah Connolly

SUNY Upstate Medical University

Hannah Cunningham

Duke University School of Medicine

Sanjana Dayananda

University of Pittsburgh School of Medicine

Amanda Farrell

Duke University School of Medicine

Julia Gelissen

Warren Alpert Medical School at Brown

University

Mary Gwin

University of North Caroline at Chapel Hill

Lauren Himes

Wake Forest School of Medicine

Alice Huang

Warren Alpert Medical School at Brown

University

Austin Jones

Tulane University

Savannah Karmen-Tuohy

New York University School of Medicine

Maya Ladenheim

University of California, San Francisco

Roger Lin

University of Maryland School of Medicine

Breeanna Lorenzen

University of Minnesota

Jez Marston

Weill Cornell Medical College

Aislinn McMillan

University of California, San Diego

John Mershon

University of Virginia School of Medicine

Anushay Mistry

University of Massachusetts

Marissa Nahirney

University of Alberta

Cody Nelson

Duke University School of Medicine

Subhjit Sekhon

University of Missouri-Kansas City

Amaya Wittmaack

University of Virginia School of Medicine
Kristen Zozulin

Frank H. Netter, MD School of Medicine at Quinnipiac University

2018

George Agyapong

Harvard Medical School

Nahid Bakhtari

Stony Brook School of Medicine

Diana Cheung

NYIT College of Osteopathic Medicine

Daniel Farrell

SUNY Upstate Medical University

Rose Gabert

University of Washington School of Medicine

George Hafzalla

Wake Forest School of Medicine

Sardis Harward

University of Massachusetts Medical School

Rebecca Henderson

University of Florida

Daniel Hodson

Yale School of Medicine

Diana Jeang

Emory University School of Medicine

Cesar Lopez

University of North Carolina

Raffaele Macri

Ross University School of Medicine

Matthew Neale

Warren Alpert Medical School of Brown University

Joseph Perosky

Michigan State University College of Human Medicine

Fartoon Siad

University of Calgary

Michael Sikorski

University of Maryland School of Medicine

Shelby Stoneking

University of North Carolina

Margaret To

Emory University School of Medicine

Grace Trompeter

Jacobs School of Medicine and Biomedical Sciences at University of Buffalo

Malcolm Velasco

Mercer University School of Medicine

Lisa Wanda

University of North Carolina

2017

Mustafa Abid

Wake Forest University School of Medicine

Precious Anyaoha

Morehouse School of Medicine 


\section{BENJAMIN H. KEAN TRAVEL FELLOWSHIP IN TROPICAL MEDICINE (cont.)}

Brandon Berger

University of Chicago Pritzker School of Medicine

Nicholas Brazeau

University of North Carolina at Chapel Hill School of Medicine

Catherine Castro

University of Chicago Pritzker School of Medicine

Shilpa Darivemula

Albany Medical College

Jessica Dawson

University of Washington

John Diehl

Emory University School of Medicine

Celia Fung

University of Rochester School of Medicine and Dentistry

Paris Hantzidiamantis

State University of New York

Megan Harris

SUNY Upstate Medical University

Monalisa Hassan

Wake Forest University School of Medicine

Justin Hills

University of North Carolina at Chapel Hill School of Medicine

John Kahler

State University of New York

Matthew Matson

Marshall University

Qaasim Mian

University of Alberta

Courtney Pedersen

Stanford University School of Medicine

Julia Ramos

Johns Hopkins University School of Medicine

Margaret Robinson

Stanford University School of Medicine

Tu Tran

University of Minnesota

Hannah Wild

Stanford University School of Medicine

\section{6}

Meagan Barry

Baylor College of Medicine

Christine Bogdanoff

University of Texas Medical Branch

Jessica Eby

University of Pennsylvania

Max Feinstein

Case Western Reserve University

Michael Harper

University of Colorado School of Medicine

Adam Kley

University of Texas Medical Branch

Vinay Krupadev

Northeast Ohio Medical University

Elizabeth Larson

Albert Einstein College of Medicine
Zachary Enumah

Johns Hopkins University School of Medicine

Andrew Mullin

Wake Forest School of Medicine

Ryan Nightingale

SUNY Update Medical University

Brady Page

Tulane University School of Medicine

Kristi Ray

Nova Southeastern University College of Osteopathic Medicine

Brenna Stanczyk

University of North Carolina

Anthony Puthumana

SUNY Update Medical University

David Roberts

University of Washington

Katherine Ryken

University of Iowa, Carver College of Medicine

Tiange Zhang

Loyola University, Stritch School of Medicine

2015

Britt Andersen

Washington University School of Medicine

Ashley Appiagyei

University of North Carolina School of Medicine

Neima Briggs

University of Texas Medical School at Houston and M.D.

Anderson Cancer Center Ignacio Cerdena

Yale School of Medicine

Madison Cornwell (In Memoriam)

University of California San Diego School of Medicine

James Cotton

University of Calgary

Andrew Flynn

University of Colorado School of Medicine

David Guernsey

NYIT-College of Osteopathic Medicine

Bryna Harrington

University of North Carolina at Chapel Hill School of

Medicine

Jo Henderson-Frost

Albert Einstein College of Medicine

Brenden Jenks

Case Western Reserve University School of Medicine

Brandon Kuang

University of California at Los Angeles

Amos Lichtman

University of Massachusetts Medical School, Harvard

School of Public Health

Nathan Lo

Stanford University School of Medicine

Erica Ludi

Emory University

Haiyan Ramirez Batlle

Alpert Medical School of Brown University 


\section{BENJAMIN H. KEAN TRAVEL FELLOWSHIP IN TROPICAL MEDICINE (cont.)}

Kelsey Ripp

Warren Alpert Medical School at Brown University

David Sanchez

Howard University College of Medicine

Milan Satcher

The Warren Alpert Medical School of Brown University

David Schaffer

University of North Carolina at Chapel Hill

Adrianna Stanley

Dartmouth Medical School

2014

Michelle Beam

Oregon Health \& Science University

Richard Broadhurst

University of North Carolina School of Medicine

William Bruno

University of California San Diego School of Medicine

Katrina Ciraldo

Boston University

Amelia Cline

University of North Carolina School of Medicine

Alex Cortez

University of Cincinnati College of Medicine

Ian Eisenhauer

University of Colorado School of Medicine

Shekinah Elmore

Harvard Medical School

Kimberly Faldetta

Pennsylvania State University College of Medicine

Morgan Goheen

University of North Carolina at Chapel Hill

Jill Hagey

University of California San Francisco

Seth Hoffman

Medical School for International Health (Ben-Gurion

University in affiliation with Columbia University)

June-Ho Kim

Johns Hopkins University School of Medicine

Chelsea McCullough

Emory University School of Medicine

Erika Phelps

Michigan State University

Nathaniel Rogers

University of Alabama at Birmingham School of

Medicine

Jonathan Schultz

University of lowa Carver College of Medicine

Stephen Selinsky

Michigan State University College of Human Medicine

Daniel Silva

Boston University School of Medicine

Bianca Stifani

Alpert Medical School of Brown University

Austin Wesevich

Washington University School of Medicine
Ya Zhou

University of Maryland School of Medicine

2013

Ameer Abutaleb

University of Maryland

Rachel Blake

Alpert Medical School of Brown University

Ethan Bornstein

Emory University School of Medicine

Sarah Boudova

University of Maryland

Chelsea Canon

Wake Forest School of Medicine

Mark Carlberg

SUNY Upstate Medical University

Aubri Carman

The University of Arizona College of Medicine

Seth Congdon

University of North Carolina School of Medicine

Melvin Donaldson

University of Minnesota

Sam Du

University of Maryland School of Medicine

Ahmar Hashmi

University of Texas Medical Branch

Marguerite Hoyler

Harvard Medical School

Daniel Huck

Case Western Reserve University School of Medicine

Genna Jerrard

University of Maryland School of Medicine

Christian Parobek

University of North Carolina Chapel Hill

Nina Prasanphanich

Emory University School of Medicine

Julia Rosenberg

Weill Cornell Medical College

Nicholas Sausen

University of Minnesota

Leah Wibecan

Harvard Medical School

Joshua Wong

Harvard Medical School

\section{2}

Jasmine Beria

New York College of Osteopathic Medicine

Geoffrey Buckle

University of Massachusetts Medical School

Jeffrey Campbell

Harvard Medical School

Jamie Carter

University of North Carolina School of Medicine

Shama Cash-Goldwasser

McGill University 


\section{BENJAMIN H. KEAN TRAVEL FELLOWSHIP IN TROPICAL MEDICINE (cont.)}

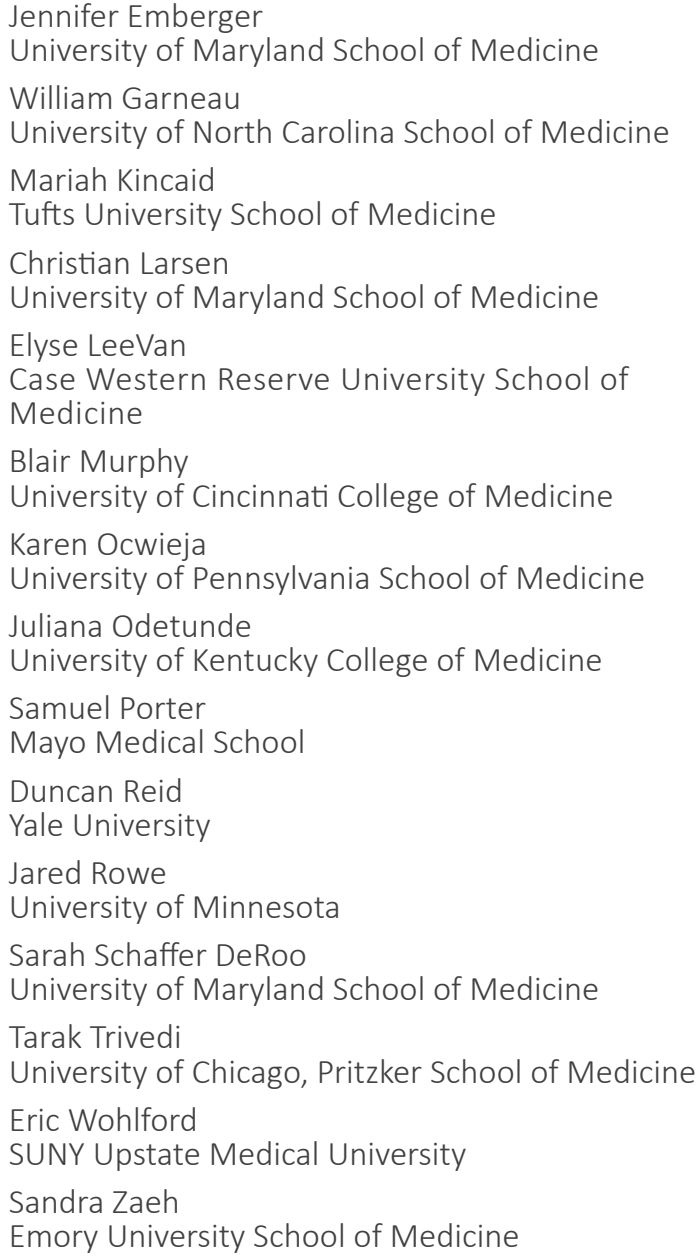

\section{1}

Jonathan Abelson

University of Virginia

Jonathan Abraham

Harvard University Medical School

Ufuoma Akoroda

Duke University School of Medicine

Andrew Bouley

Duke University

Nicholas Carter

Brown University Warren Alpert Medical School

Whitney Harrington

University of Washington

Nicholas Janusz

University of Manitoba

Ellen Jones

University of Texas Southwestern Medical School

Brian Long

University of Virginia

Micah Manary

University of California, San Diego

Emma Mohr

University of lowa Carver College of Medicine
Connor O'Brien

Columbia University College of Physicians and Surgeons

Evan Orenstein

Emory University School of Medicine

Amelie Peryea

University of Washington

Trisha Schimek

Jefferson Medical College

Jennifer Spicer

Emory University School of Medicine

Carla Valenzuela

Vanderbilt University

Sarah Ventre

SUNY Upstate Medical University

Jacqueline Weiss

University of California at San Francisco Medical School

Emily Wilkinson

University of Arizona College of Medicine

2010

Malika Atmakuri

Pennsylvania State University College of Medicine

Brian Bergmark

Harvard Medical School

Regan Bergmark

Harvard Medical School

Andrew Brookens

University of Colorado School of Medicine

Katharine Burns

Vanderbilt School of Medicine

Nicole Cooper

Baylor College of Medicine/Harvard

School of Public Health

Benjamin Cunningham

New York University School of Medicine

Marianna Freudzon

Yale School of Medicine

Eric Kalivoda

University of Vermont College of Medicine

Charles Langelier

University of Utah

Jennifer Manne

Boston University School of Medicine

Jonathan Merola

New York University School of Medicine

Juliana Minak

University of Virginia

Aaloke Mody

Duke University School of Medicine

Tafadzwa Muguwe

Harvard Medical School

Scott Nabity

Duke University School of Medicine

Heyman Oo

University of California San Diego School of Medicine 


\section{BENJAMIN H. KEAN TRAVEL FELLOWSHIP IN TROPICAL MEDICINE (cont.)}

Adi Price

University of California San Diego School of Medicine

Joanne Smucker

Pennsylvania State University College of Medicine

Ailis Tweed-Kent

Harvard Medical School

\section{9}

Sara Janne Aarsland

University of Texas Medical Branch

Byron Berenger

University of Calgary

Rachel Bystritsky

Mount Sinai School of Medicine

Youngjee Choi

Washington University School of Medicine

L. Emily Carter

George Washington University

Dylan Davey

Yale University

Qyana Griffith

Boston University School of Medicine

Jenny Hsu

University of Vermont College of Medicine

Alexis Kearney

Mount Sinai School of Medicine

Kristina Krohn

University of Minnesota

Rodwell Mabaera

Darmouth Medical School

Tracy Mak

Weill Cornell Medical College

Marilyn Michelow

Weill Cornell Medical College

Grace Milad

University of Virginia School of Medicine

Molly Paras

Mayo Clinic College of Medicine

Barclay Stewart

Medical University of South Carolina

Scott Tolan

University of Texas School of Medicine at Houston

\section{8}

Rushina Cholera

University of North Carolina, Chapel Hill

Cassidy Claassen

University of Maryland

Jeffrey Collins

Case Western Reserve University

Ryan Dare

University of Arkansas for Medical Sciences

William Hahn

SUNY Upstate Medical University

Alison Han

Morehouse School of Medicine
Anna Yasmine Kirkorian

Mount Sinai School of Medicine

Rebecca Lawniczak

Vanderbilt University Medical School

Fiona Lovegrove

University of Toronto

Anna Minta

Stanford University School of Medicine

Kimberly Rutherford

McMaster University

Meaghann Weaver

University of Nebraska Medical Center

2007

Cullen Carter

University of Virginia

Heidi Hillesland

University of New Mexico

Atis Muehlenbachs

University of Washington

Sean Murphy

Northwestern University

Eugene Richardson

Weill Cornell Medical College

\section{6}

Karl Bezak

Vanderbilt University

Kara Goss

Baylor College of Medicine

Victoria Kuohung

Yale University Alyssa Lovell University of Iowa

Douglas Olson

George Washington University

Christina Polyak

University of Maryland

Heather Strah

University of lowa

Luisa Stramm

University of California, San Francisco

Marisa Wagner

Harvard Medical School

Louise Vaz

Vanderbilt University

\section{5}

Sarah Atunah-Jay

Brown University Kathryn Duphik University

of Virginia

Megan Leo

University of Florida

Sarah Meyer

St. Louis University

Megan Morsheimer

University of California at Los Angeles

Amy Oyler

University of Virginia 


\section{BENJAMIN H. KEAN TRAVEL FELLOWSHIP IN TROPICAL MEDICINE (cont.)}

\author{
Irene Sarantakos \\ Tulane University \\ Alexander Tsai \\ Case Western Reserve University \\ Michael Westerhaus \\ Harvard University \\ Christine Zink \\ Cornell University

\section{4} \\ Ben Andrews \\ Baylor College of Medicine \\ Daniel Leung \\ Wake Forest University \\ Kevin Lunney \\ University of Maryland School of Medicine \\ Heidi Sandige \\ Washington University \\ Zongqui Xia \\ Case Western Reserve University

\section{3} \\ Sujeet Acharya \\ Baylor College of Medicine \\ Supinda Bunyavanich \\ Harvard University Medical Schoo \\ David Edwards \\ Duke University School of Medicine \\ Jeanne Noble \\ University of California, San Francisco
}

\section{2}

David B. Blossom

University Hospitals of Cleveland

Katrina Gray

Albany Medical College

Ingrid Lundgren

Mayo Medical School

Mehret Mandefro

Harvard Medical School

Sean Moore

Johns Hopkins University

\section{1}

Peter Crompton

Massachusetts General Hospital

Amy Dechet

University of California, San Francisco

Meg Doherty

Massachusetts General Hospital
William Fischer

University of North Carolina, Chapel Hill

Danny Milner

Brigham and Women's Hospital

Tuan Tran

Emory University

2000

Sona Aggarwal

Johns Hopkins University

Annie-Claude

Labbe Laval University

Nina Lin

Harvard Medical School

Iyesatta Massquio

Case Western Reserve University

Katrina McPherson

Johns Hopkins University School of Medicine

Holly Murphy

Tulane University

Tina Rutar

Harvard Medical School

David Walton

Harvard Medical School

\section{9}

Rachel Ash-Bernal

Medical College of Pennsylvania-Hahnemann

Richard Brantley

University of Virginia

Carol McLaughlin

Johns Hopkins University School of Medicine

Rebecca Plank

University of California at San Francisco

Paul Robben

Washington University School of Medicine

Amil Shah

University of Pennsylvania School of Medicine

\section{8}

Stephen Altmin

Vanderbilt University

Michele Morse

Johns Hopkins University

Dylan Pillai

University of Toronto

Allison Portney

Yale University 


\section{GORGAS MEMORIAL INSTITUTE RESEARCH AWARD (2014 - final year of award)- This award was designed to enhance and facilitate the development of scientific link- ages between Panama, nations of Central America and the Caribbean Islands and the United States and Canada through support of short-term travel for young research investigators from these regions.}

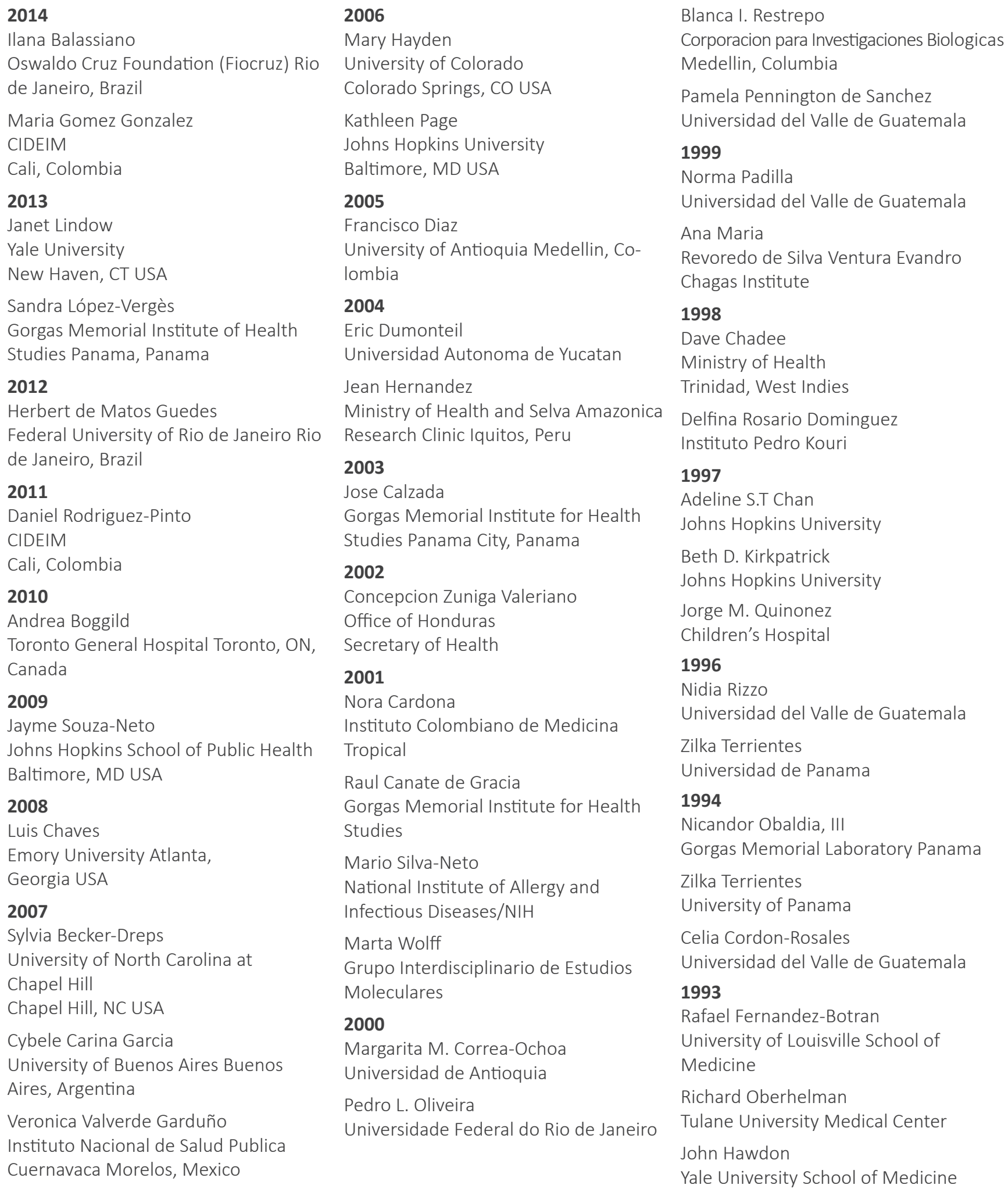


ASTMH FELLOWSHIP IN TROPICAL MEDICINE AND TROPICAL PUBLIC HEALTH-

This award supports a one-year postdoctoral fellowship to provide field experience in endemic areas for individuals working on tropical infectious disease-related research in the U.S. (1999final year of award).

\section{9}

Kevin P. Fennelly

National Jewish Medical and Research Center

1996

Benjamin Wizel-Cohen

University of Georgia

1998

Peter McElroy

Centers for Disease Control
1995

Jonathan Kurtis

Brown University

1996

Christine Muller-Graf

University of Virginia

1994

Nicholas Anstey

Duke University

\section{YOUNG INVESTIGATOR AWARD-}

Each year, ASTMH recognizes the work of outstanding young investigators through the presentation of the Young Investigator Awards, a yearly competition held during the first day of the Annual Meeting. This award encourages young scientists to pursue careers in various aspects of tropical disease research.

\section{Winners}

Alexandra Ehrens . . . . . . . . . . . . 2019

Beatriz Galatas . . . . . . . . . . . . . . . 2019

Maria Simoes ............... . 2019

Hannah Steinberg . . . . . . . . . . . . 2019

Kristine Werling. . . . . . . . . . . 2019

First Tier Mention

Kristyn Hoffman ... . . . . . . . . . . . 2019

Paulo Manrique Valverde. . . . . . . . . . . 2019

Catherine Mitran . . . . . . . . . . . . 2019

Talia Quandelacy. . . . . . . . . . . . . . . . . 2019

Alyse Wheelock. ................ 2019

\section{Honorable Mention}

Marco Brustolin . . . . . . . . . . . . . . 2019

Cristina Costales . . . . . . . . . . . . . . . . 2019

Santosh George. . . . . . . . . . . . . . . . . 2019

Brien Haun. . . . . . . . . . . . . . . . . . . . . 2019

Yvett Sosa . . . . . . . . . . . . . . . . . . . . 2019

Winners

Cordelia Coltart. . . . . . . . . . . . . . . . 2018

Ethan Degner . . . . . . . . . . . . . . . . . . 2018

Christina Faust. . . . . . . . . . . . . . . . . 2018

Erin MacDonald . . . . . . . . . . . . . 2018

Catherine Mitran . . . . . . . . . . . . . . . 2018

First Tier Mention

Ian Buller. . . . . . . . . . . . . . . . . . 2018

Pedro Gazzinelli-Guimar. . . . . . . . . . . . 2018

Karthigayan Gunalan . . . . . . . . . . . . 2018

Julia Mwesigwa. . . . . . . . . . . . . . . . . . . 2018

Olivia Winokur . . . . . . . . . . . . . . 2018

\section{Honorable Mention}

Sonia Agrawal . . . . . . . . . . . . . . . . . . . 2018

Johannes Mischlinger. . . . . . . . . . . . . 2018

Niraja Suresh . . . . . . . . . . . . . . . . . . . 2018

Luzia Veletzky . . . . . . . . . . . . . . . . . . . 2018

Megan Vogt. . . . . . . . . . . . . . . . . . 2018

\section{Winners}

Alexandra Grote . . . . . . . . . . . . . . . . 2017

Martina Laidemitt. . . . . . . . . . . . . . . . . . 2017

Inke Lubis. . . . . . . . . . . . . . . . . . . . 2017

David Markman. . . . . . . . . . . . . . . . . . 2017

Katelyn Walzer . . . . . . . . . . . . . . . 2017

First Tier Mention

David Berendes. . . . . . . . . . . . . . . . . . 2017

Charlotte Heuvelings . . . . . . . . . . . . . 2017

Hyeogsun Kwon . . . . . . . . . . . . . . . 2017

Jiangtao Liang . . . . . . . . . . . . . . . . . . 2017

Carla Mavian . . . . . . . . . . . . . . . . . 2017

Honorable Mention

Manuela Carugati . . . . . . . . . . . . . . . 2017

Sage Davis . . . . . . . . . . . . . . . . . . 2017

Kenneth Gavina. . . . . . . . . . . . . . . . . . 2017

Raul Saraiva . . . . . . . . . . . . . . . . . . 2017

Angela Toepp. . . . . . . . . . . . . . . . . . 2017

\section{Winners}

Nicholas Bergren . . . . . . . . . . . . . 2016

Bethany Caruso . . . . . . . . . . . . . . . . 2016

Richard Davis. . . . . . . . . . . . . . . . . . 2016

Kristina Keitel . . . . . . . . . . . . . . . 2016

Kara Moser . . . . . . . . . . . . . . . . . . 2016

First Tier Mention

Suzy Campbell. . . . . . . . . . . . . . . . . 2016

Morgan Goheen . . . . . . . . . . . . . . . . . . 2016

John Jimah. . . . . . . . . . . . . . . . . . . 2016

Christine Markwalter . . . . . . . . . . . . . . 2016

Deepali Ravel . . . . . . . . . . . . . . . 2016

\section{Honorable Mentions}

Gunjan Arora. . . . . . . . . . . . . . . . . 2016

Sarah Buddenborg . . . . . . . . . . . . . . . . . . . 2016

Sung-Jae Cha . . . . . . . . . . . . . . . . . . . . . . 2016

Breanna Scorza . . . . . . . . . . . . . . . . . . . 2016

Donna Tyungu. . . . . . . . . . . . . . . . . 2016 


\section{YOUNG INVESTIGATOR AWARD (cont.)}

\section{Winners}

Melanie Abongwa. . . . . . . . . . . . . . . . . 2015

Sarah Short . . . . . . . . . . . . . . . . 2015

Hugo Valdivia. . . . . . . . . . . . . . . . . 2015

Brandi Freeman . . . . . . . . . . . . . . . . . 2015

Emily Gallichotte. . . . . . . . . . . . . . . . . 2015

\section{First Tier Mention}

Sarah Boudova ................... 2015

Brandyce St. Laurent . . . . . . . . . . . . . . 2015

Richard Pinapati . . . . . . . . . . . . . . . 2015

Estela Shabani . . . . . . . . . . . . . . . . . . . . 2015

Jesse Erasmus . . . . . . . . . . . . . . . . 2015

\section{Honorable Mentions}

Andreea Waltmann ..... . . . . . . . . . . 2015

Albert Jonathan Auguste . . . . . . . . . . . . 2015

Samantha Nava. . . . . . . . . . . . . . . . . 2015

Jagrati Jain . . . . . . . . . . . . . . . . . . . . . . 2015

Rahajeng Tunjungputri. . . . . . . . . . . 2015

\section{Winners}

Kayla Barnes . . . . . . . . . . . . . . . . . . . . . 2014

Allison Demas . . . . . . . . . . . . . . . . . . . . 2014

Carmen Dickinson-Copeland . . . . . . . . 2014

Anna Last. . . . . . . . . . . . . . . . . . . . . . . . 2014

Abdallah Samy. . . . . . . . . . . . . . . . . . . 2014

\section{First Tier Mention}

Francesco Baldini . . . . . . . . . . . . . . . . . 2014

Augusto Carvalho . . . . . . . . . . . . . . . . . . 2014

Emanuele Giorgi . . . . . . . . . . . . . . . . . . . 2014

Serene Joseph. . . . . . . . . . . . . . . . . . . . 2014

Simon Pollett. . . . . . . . . . . . . . . . . . 2014

\section{Honorable Mentions}

Fiona Angrisano . . . . . . . . . . . . . . . . 2014

Jessica Atwell. . . . . . . . . . . . . . . . . . . . . . 2014

Katia Bruxvoort . . . . . . . . . . . . . . . . . 2014

Jessica Hostetler . . . . . . . . . . . . . . . . . . 2014

Eugene Liu . . . . . . . . . . . . . . . . 2014

\section{Winners}

Aaron Neal. . . . . . . . . . . . . . . . . . . . . . 2013

Fernando Pereira Bruno. . . . . . . . . . . . . . 2013

Michael Smouth . . . . . . . . . . . . . . . . . . 2013

Derek Trobaugh. . . . . . . . . . . . . . . . . . . 2013

Rakiswendé Yerbanga. . . . . . . . . . . . . 2013

\section{First Tier Mention}

Sheila Akinyi . . . . . . . . . . . . . . . . . . . . . 2013

Cayley Bowles . . . . . . . . . . . . . . . . . . . 2013

Martha Clark . . . . . . . . . . . . . . . . . . . . 2013

Richard Davis. . . . . . . . . . . . . . . . . . . 2013

Joseph Wagman . . . . . . . . . . . . . . 2013

\section{Honorable Mentions}

Ahmed Abd El Wahed . . . . . . . . . . . . . 2013

Giridhar Athrey . . . . . . . . . . . . . . . . . . 2013

Sethu Nair . . . . . . . . . . . . . . . . . . . . . . 2013

Richard Sullivan. . . . . . . . . . . . . . . . . 2013

Tuan Tran. . . . . . . . . . . . . . . . . . . . 2013

\section{Winners}

Jennifer Armistead . . . . . . . . . . . . . . . 2012

Kovi Bessoff. . . . . . . . . . . . . . . . . . . 2012
Holly Evans . . . . . . . . . . . . . . . . . 2012

Jen Manne. . . . . . . . . . . . . . . . . . . . . . . 2012

Nana Wilson . . . . . . . . . . . . . . . . . 2012

First Tier Mention

Bridget Barber. . . . . . . . . . . . . . . . 2012

Andrew Bouley . . . . . . . . . . . . . . . . . . . 2012

Alexander Douglas . . . . . . . . . . . . . 2012

Alexander Narty . . . . . . . . . . . . . . . . . . 2012

Sanie Sesay . . . . . . . . . . . . . . . . . . . . 2012

\section{Honorable Mentions}

Miranda Delahoy . . . . . . . . . . . . . . . 2012

Assem Harith Hamed Al-Ani . . . . . . . . . 2012

Poonum Korpe . . . . . . . . . . . . . . . 2012

Tchouassi Poumo . . . . . . . . . . . . . . . . 2012

Geoffrey H. Siwo . . . . . . . . . . . . . . . 2012

\section{Winners}

Joshua Benoit . . . . . . . . . . . . . . . . . . 2011

Shevaun Davis . . . . . . . . . . . . . . . . . . . 2011

Michael Lo. . . . . . . . . . . . . . . . . . . 2011

Ricardo J. Soares Magalhaes . . . . . . . . . 2011

Joaniter NanKabirwa . . . . . . . . . . . . . 2011

\section{First Tier Mention}

Mark Gillrie . . . . . . . . . . . . . . . . . . . . . 2011

Julian Hertz . . . . . . . . . . . . . . . . . . . . 2011

Daniel T. Leung . . . . . . . . . . . . . . . . . 2011

Maggy Sikulu . . . . . . . . . . . . . . . . . . . . . 2011

Stephanie Zimmerman. . . . . . . . . . . . . 2011

\section{Honorable Mentions}

Kelly Baker. . . . . . . . . . . . . . . . . . . 2011

Joanne Berghout. . . . . . . . . . . . . . . . . 2011

Maryam Kimali . . . . . . . . . . . . . . . . . 2011

Margaret Mccoy . . . . . . . . . . . . . . . . . 2011

Sarah Wheeler ............... 2011

\section{Winners}

Michelle Boyle. . . . . . . . . . . . . . . . . . 2010

Christopher Cirimotich . . . . . . . . . . . . 2010

Andrea Conroy . . . . . . . . . . . . . . . . 2010

Yeung Lo. . . . . . . . . . . . . . . . . . . . . . . 2010

Yovany Moreno . . . . . . . . . . . . . . . . . 2010

\section{Runners-Up}

Matthew Aliota . . . . . . . . . . . . . . . . 2010

Nadine Riedel . . . . . . . . . . . . . . . . . . . 2010

JB Sarr . . . . . . . . . . . . . . . . . 2010

Tara Thiemann . . . . . . . . . . . . . . . . . 2010

Elizabeth Villasis . . . . . . . . . . . . . . . . 2010

\section{Honorable Mentions}

Lauren Cator . . . . . . . . . . . . . . . . . . 2010

Christine Ferragine . . . . . . . . . . . . . . 2010

Guilherme Ribeiro. . . . . . . . . . . . . . 2010

Claudia Valentim . . . . . . . . . . . . . . . . . 2010

Jordan Zuspann. . . . . . . . . . . . . . . . . . 2010

\section{Winners}

Joanne T. Baker . . . . . . . . . . . . . . . . 2009

Whitney E. Harrington . . . . . . . . . . . . . 2009

Tamaki Kobayashi . . . . . . . . . . . . . . . . . 2009

Fredros O. Okumu. . . . . . . . . . . . . . . . 2009

Mark A. Wacker. . . . . . . . . . . . . . . . . . 2009 


\section{YOUNG INVESTIGATOR AWARD (cont.)}

\section{Runners-Up}

Lauren J. Cator . . . . . . . . . . . . . . . . 2009

Hannah Chiu . . . . . . . . . . . . . . . . . . 2009

Rachel Beaulieu Goldsmith . . . . . . . . . . 2009

Brett E. Swierczewski . . . . . . . . . . . . . 2009

Sumiti Vinayak. . . . . . . . . . . . . . . . . . 2009

\section{Honorable Mentions}

Allison C. Brown . . . . . . . . . . . . . . . . . 2009

Sarah J. Higgins . . . . . . . . . . . . . . . . 2009

Heidi Hillesland . . . . . . . . . . . . . . . . . . 2009

David Larson . . . . . . . . . . . . . . . . . . . 2009

Jason M. Meyer. . . . . . . . . . . . . . . . . 2009

\section{Winners}

Jacqueline Janka . . . . . . . . . . . . . . . . . 2008

Samantha McNulty. . . . . . . . . . . . . . . 2008

Agnes Mwakingwe . . . . . . . . . . . . . . . . 2008

Meera Venkatesan . . . . . . . . . . . . . . . 2008

Shuyi Zhang. . . . . . . . . . . . . . . . . 2008

\section{Runners-Up}

Pamela Orjuela-Sánchez . . . . . . . . . . . 2008

James Potts . . . . . . . . . . . . . . . . . . . 2008

Jennifer Simpson. . . . . . . . . . . . . . . . . . 2008

Kristin Smith . . . . . . . . . . . . . . . . . . 2008

Prakash Srinivasan . . . . . . . . . . . . . . 2008

\section{Honorable Mention}

Charles Criscione . . . . . . . . . . . . . . . 2008

Julio Croda. . . . . . . . . . . . . . . . . . . . 2008

David Larson . . . . . . . . . . . . . . . . . . 2008

Edwin Ochong. . . . . . . . . . . . . . . . . 2008

Nikos Vasilakis . . . . . . . . . . . . . . . . . . . . 2008

\section{Winners}

Arlene Dent. . . . . . . . . . . . . . . . . . . . 2007

Claire Marie Filone . . . . . . . . . . . . . . 2007

Godfree Mlambo . . . . . . . . . . . . . . . . 2007

Lisa Purcell. . . . . . . . . . . . . . . . . . . . . . 2007

Michelle Steinauer . . . . . . . . . . . . . . . . 2007

\section{Honorable Mention}

Christine Hsiao . . . . . . . . . . . . . . . . . . 2007

Marc Hübner. . . . . . . . . . . . . . . . . . . 2007

Nadia Sam-Agudu . . . . . . . . . . . . . . . . 2007

Kristin Smith ... . . . . . . . . . . . . . . . 2007

Shannon Takala. . . . . . . . . . . . . . . . . . 2007

\section{Winners}

Matthieu Gissot . . . . . . . . . . . . . . . . . . 2006

Adrienne Meyers . . . . . . . . . . . . . . 2006

Jennifer Spence. . . . . . . . . . . . . . . . . . 2006

Robert Tweyongyere . . . . . . . . . . . . . . 2006

\section{Honorable Mention}

Brian Grimberg . . . . . . . . . . . . . . . . . 2006

Naomi Lucchi. . . . . . . . . . . . . . . . . . . . 2006

Robin Moudy. . . . . . . . . . . . . . . . . . 2006

Kristina Persson. . . . . . . . . . . . . . . . . . 2006

\section{Winners}

Marina Allary. . . . . . . . . . . . . . . . . . . . . 2005

Michael Donovan . . . . . . . . . . . . . . 2005

Amara Ezeamama. . . . . . . . . . . . . . . . . 2005

Christine Hice . . . . . . . . . . . . . . . . . . 2005

\section{Honorable Mention}

Janet Achan. .......................... 2005

Uzma Alam . . . . . . . . . . . . . . . . . . 2005

Kate McElroy . . . . . . . . . . . . . . . . . . . . . 2005

Ahmed Sayed . . . . . . . . . . . . . . . . . . . 2005

\section{Winners}

Laurin Kasehagen . . . . . . . . . . . . . . . 2004

Kimberly Keene. . . . . . . . . . . . . . . . . . . . 2004

Kim-Sung Lee . . . . . . . . . . . . . . . . . . 2004

Nilda Rodriguez. . . . . . . . . . . . . . . . . . . 2004

\section{Honorable Mention}

Alisa Alker . . . . . . . . . . . . . . . . . . . . . . 2004

Jennifer Anderson. . . . . . . . . . . . . . . 2004

Renee Larocque . . . . . . . . . . . . . . . . . . . 2004

Charlotte Lanteri. . . . . . . . . . . . . . . . . 2004

\section{Winners}

Michael Brown . . . . . . . . . . . . . . . . 2003

Rick Fairhurst. . . . . . . . . . . . . . . . . . . 2003

Ivorlyne Greene . . . . . . . . . . . . . . . . 2003

Anne Schwab. . . . . . . . . . . . . . . . . . . . 2003

\section{Honorable Mention}

Alfred Cortes. . . . . . . . . . . . . . . . . . . . 2003

Kari Gillenwater. . . . . . . . . . . . . . . . . 2003

Illona Gillette-Ferguson . . . . . . . . . . . . 2003

Elmer Saathoff . . . . . . . . . . . . . . . . 2003

\section{Winners}

Subash Babu . . . . . . . . . . . . . . . . . 2002

Victor Mwapasa . . . . . . . . . . . . . . . . . . 2002

Salima Nathoo. . . . . . . . . . . . . . . . . . 2002

Boonrat Tassaneetrithep . . . . . . . . . . . 2002

\section{Honorable Mention}

Kai-Hsin Chang . . . . . . . . . . . . . . . . . . . 2002

Ali Dana . . . . . . . . . . . . . . . . . . . . 2002

Laura Goddard . . . . . . . . . . . . . . . . . 2002

Amy Hise . . . . . . . . . . . . . . . . . . . . 2002

Sandra Laney. . . . . . . . . . . . . . . . . . . . 2002

Edward Mitre . . . . . . . . . . . . . . . . . . . 2002

\section{Winners}

Craig Boutlis . . . . . . . . . . . . . . . 2001

Meghna Desai . . . . . . . . . . . . . . . . . . . . . 2001

Avelina Espinosa . . . . . . . . . . . . . . . . . . 2001

Jason Rasgon. . . . . . . . . . . . . . . . . . . . 2001

\section{Honorable Mention}

Kai-Hsin Chang . . . . . . . . . . . . . . . . . . . 2001

Andrea Crampton . . . . . . . . . . . . . . . 2001

Matthew Dodge . . . . . . . . . . . . . . . . . 2001

Heather Ferguson . . . . . . . . . . . . . . . . 2001

Iveth Gonzalez. . . . . . . . . . . . . . . . . . . . . 2001

Lillian Ogonda . . . . . . . . . . . . . . . . . . . . 2001

Sheral Patel . . . . . . . . . . . . . . . . . 2001

Eduardo Villamor . . . . . . . . . . . . . . 2001

\section{Winners}

Zach Adelman . . . . . . . . . . . . . . . . . . . 2000

Gregory Anstead. . . . . . . . . . . . . . . . . 2000

Oralee Branch . . . . . . . . . . . . . . . . . . . 2000

Molly Hughes . . . . . . . . . . . . . . . . . . 2000

Julian Rayner. . . . . . . . . . . . . . . . . . . . 2000 


\section{YOUNG INVESTIGATOR AWARD (cont.)}

\section{Honorable Mention}

Aaron Brault . . . . . . . . . . . . . . . . . . . 2000

Kira Gantt. . . . . . . . . . . . . . . . . . . . . 2000

Hai Qi . . . . . . . . . . . . . . . . . . . . . . . . 2000

Lena Serghides . . . . . . . . . . . . . . . . . 2000

Susan Thomas... . . . . . . . . . . . . . 2000

\section{Winners}

Tedros Ghebreyesus . . . . . . . . . . . . . . . 1999

Christopher Huston . . . . . . . . . . . . . 1999

Audrey Lau . . . . . . . . . . . . . . . . . . . . 1999

P'ng Loke . . . . . . . . . . . . . . . . . . . . . . 1999

Elena Pollina . . . . . . . . . . . . . . . . 1999

\section{Honorable Mention}

Serge Ankri . . . . . . . . . . . . . . . . . . . . . 1999

Peter Blair . . . . . . . . . . . . . . . . . . . . . . 1999

Antonio Del Valle ... . . . . . . . . . . . . . 1999

Seydou Doumbia. . . . . . . . . . . . . . . . . . 1999

Kira Gantt. . . . . . . . . . . . . . . . . . . . . 1999

Karen Myung. . . . . . . . . . . . . . . . . . . . 1999

Lena Serghides . . . . . . . . . . . . . . . . . 1999

Tom Solomon . . . . . . . . . . . . . . . . . . 1999

\section{Winners}

Stefan Kappe . . . . . . . . . . . . . . . . . . . . . 1997

Manuel Moro . . . . . . . . . . . . . . . . . . . 1997

Sacha Sidjanski . . . . . . . . . . . . . . . . . . 1997

\section{Honorable Mention}

Gabriel Bonilla. . . . . . . . . . . . . . . . . . . 1997

Richard Bungiro, Jr. . . . . . . . . . . . . . 1997

Michael Ferdig . . . . . . . . . . . . . . . . . 1997

Carol Othoro . . . . . . . . . . . . . . . . . 1997

Lena Serghides . . . . . . . . . . . . . . . . . . 1997

\section{Winners}

Esther Dechant . . . . . . . . . . . . . . . . . 1996

Upinder Singh . . . . . . . . . . . . . . . 1996

Jason Wooden. . . . . . . . . . . . . . . . . . . 1996

\section{Honorable Mention}

Jackeline Alger. . . . . . . . . . . . . . . . . . 1996

Kim Halpin . . . . . . . . . . . . . . . . . . . . . 1996

Elaine Rodrigues . . . . . . . . . . . . . . . . . . . 1996

Edward Ryan . . . . . . . . . . . . . . . . . 1996

\section{Winners}

Larry Chow . . . . . . . . . . . . . . . . . . . . 1995

Chris Leptak. . . . . . . . . . . . . . . . . . . . 1995

Janet Yee . . . . . . . . . . . . . . . . . . 1995

\section{Honorable Mention}

Richard Brigandi . . . . . . . . . . . . . . . . . 1995

Denise Doolan. . . . . . . . . . . . . . . . . 1995

Ravi Durvasula. . . . . . . . . . . . . . . . . 1995

Benjamin Wizel . . . . . . . . . . . . . . . 1995

\begin{abstract}
Winners
Susan Gagnon . . . . . . . . . . . . . . . . . . . . . 1994

Julie Moore . . . . . . . . . . . . . . . . . . . . . . 1994

Thomas Streit . . . . . . . . . . . . . . . . . . . . 1994

Sarah Volkman . . . . . . . . . . . . . . . . . . 1994

Julio C. Rojas . . . . . . . . . . . . . . . . . . . 1994

\section{Winners}

Basile Kollo . . . . . . . . . . . . . . . . . . . . . 1993

Sigrid C. Roberts . . . . . . . . . . . . . . . . 1993

Michael Cappello . . . . . . . . . . . . . . . 1993

\section{Winners}

James J. McCoy. . . . . . . . . . . . . . . . . . . . 1992

Linette Rodriguez-Figueroa . . . . . . . . . 1992
\end{abstract}

\section{Winners}

Matthias Leippe . . . . . . . . . . . . . . . . . . 1991

Rodolfo Acuna-Soto . . . . . . . . . . . . . . . . . . 1991

\section{Winners}

Sanjai Desai . . . . . . . . . . . . . . . . . . . . 1990

Ajit P. Limaye . . . . . . . . . . . . . . . . . . . . . 1990

\section{Winners}

Ellen Kruger. . . . . . . . . . . . . . . . . . . . 1989

Misha Pless . . . . . . . . . . . . . . . . . . . . 1989

\section{Winners}

Michael Denis . . . . . . . . . . . . . . . . . 1988

Daniel R. Lucey . . . . . . . . . . . . . . . . . . . 1988

Joan Malancon-Kaplan . . . . . . . . . . . . . 1988

\section{Honorable Mention}

Thomas Eng. . . . . . . . . . . . . . . . . . 1988

L. K. Read. . . . . . . . . . . . . . . . . . . . . 1988

Tobil Yvonne Sam-Yellowe . . . . . . . . . . 1988

\section{Winners}

Terrie E. Taylor. . . . . . . . . . . . . . . . . 1987

Honorable Mention

Stephen C. Bosshardt. . . . . . . . . . . . . . 1987

\section{Winners}

William A. Petri . . . . . . . . . . . . . . . 1986

\section{Honorable Mention}

L. David Sibley . . . . . . . . . . . . . . . . . . . . . 1986

J. M. Shaw . . . . . . . . . . . . . . . . . . 1986

\section{Winner}

Ralph J. Blair . . . . . . . . . . . . . . . . 1985 
ANNUAL MEETING TRAVEL AWARD RECIPIENTS

\section{9}

Dennis Adu-Gyasi

Olugbenga Akinola

Jéssica Alves

Mireille Amba

Md Nuhu Amin

Marcos André

Martin Ayanore

Marycelin Baba

Laud Anthony Basing

Madeleine Eunice Betouke Ongwe

Jyoti Bhardwaj

Carolina Camargo

Peter Cheuka

Uchechukwu Chukwuocha

Naomi Clarke

Jennifer Clinton

Aissatou Diawara

Sandra Duffy

Elise Farley

Yalemzewod Gelaw

Alexander Gold

Oheneba Charles Hagan

Visopo Harawa

Tatiana Hountohotegbe

Trieu Huynh

Chenjerai Jairoce

Lucy John

Benson Juma

Emmanuel Kaindoa

Ruwandi Kariyawasam

Gokul Raj Kathamuthu

Aisha Khatib

Alphonso Kofa

Abibatou Konate

Ayleen Kosasih

Amy Krystosik

Titus Kwambai

David Kwasi

Viridiana Laredo-Tiscareno

Allan Lemtudo

Jonathan Liew

Jailos Lubinda

Paulo Manrique Valverde

Mikhael Manurung

Catherin Marin

Victor Mobegi

Nobert Mudare

Bushra Mufti

Shamsun Naher

Celine Nkenfou

Win Han Oo

Mercy Opiyo

Benjamin Opot

James Otieno

Rapatbhorn Patrapuvich

Lam Phung

Christiane Prosser

Gianmarco Raddi
Breanna Scorza

Robert Shaw

Robert Sumaye

Leandro Tapia

Lizzie Tchongwe

Frank Tenywa

Tshokey Tshokey

Charlene Yoboue

\section{8}

Manfred Accrombessi

Syed Fazil Ahamed

Kristen Aiemjoy

Munir Akkaya

Abena Amoah

Racheal Aya

Justy Antony Chiramal

David Cook

Eugenia Corrales-Aguilar

Alexandra Cossio

Isadora De Siqueira

Bruno Gomes

Sherry Haller

Yabo Honkpehdji

Fang Huang

Fatou Joof

J. Daniel Kelly

Damaris Matoke

Godfrey Mayoka

Oscar Mbare

Brandon Mercado

Pallavi Mishra

Jose Moreira

Beth Mutai

Julia Mwesigwa

Latif Ndeketa

Leonardo Ortega-Lopez

Alex Pauvolid-Correa

Melissa Pender

Yoenten Phuentshok

Anielle Pina Costa

Melissa Richard-Greenblatt

Peter Sangoro

Joseph Nelson Siewe Fodjo

Lwitiko Sikana

Morgan Smith

Seynabou Sougoufara

Nataya Suttanont

Dipomin Traore

Jessica Tuan

Ana Valenciano Murillo

Megan Vogt

Trung Vu

Xiaotong Zhu

2017

Adamu Addissie

Mensah Ahadji-Dabla

Tahmina Ahmed
Roberto Alcántara

Denise Alvarenga

Tiffany Borbon

Ross Boyce

Nathaniel Byers

Philip Bystrom

Manuela Carrasquilla

Emily Deichsel

Yahya Derua

Phouvieng Douangdala

Anna Fagre

Maurice Itoe

Dennis Juma

Caroline Kabaria

Donghun Kim

Sri Krishna

Inke Nadia Diniyanti Lubis

Karina Luque-Burgos

Zvifadzo Matsena

Yeromin Mlacha

Lidia Montenegro

Fathima Mubarak

Billy Ngasala

John Okombo

Olugbenga Onile

Derick Osakunor

Camilla Pires

Saravanakumar Puthupalayam Kaliappan

Binod Rayamajhee

Rasheed Salaudeen

Richard Sanya

Stacey Scroggs

Rachel Sippy

Menno Smit

Subramanian Swaminathan

Susana Vaz Nery

Hayley Yaglom

Mabot Yobo

2016

Pedro Aguiar

Shehu Awandu

Sujata Balasubramanian

Etienne Bilgo

Rebecca Brander

Guido Camargo España

Hsiao-Han Chang

Zaydah De Laurent

Michelle Dao Dong

Renato Errea

Amal Gadalla

Gabriela Garcia

Patricia Gomez-Perez

Claire Heath

Anita lyer

John Jimah

Flora Kano

Cristian Koepfli

Fanny Legrand 
ANNUAL MEETING TRAVEL AWARD RECIPIENTS (cont.)

\author{
Gathsaurie Neelika Malavige \\ Vanessa Moraes \\ Fred Mugabo \\ Mary Muhindo \\ Peninnah Muiruri \\ Patrick Mukadi Kakoni \\ Julius Mulindwa \\ Sarah Murphy \\ Ailie Robinson \\ Miryam Romano \\ Marlon Saavedra Romero \\ R. Tedjo Sasmono \\ Smriti Sharma \\ Maria Simoes \\ David Smith \\ Itziar Ubillos Escriche \\ Andrew Walakira \\ Tiendrebeogo Wendpayangde \\ Kristine Werling
}

\section{5}

Elizabeth Astupina Figueroa

Ghulam Awab

Sheila Barasa

Abebe Genetu Bayih

Georgina Bowyer

Henrietta Doe

Heven Sime Firew

Leslie Goo

Erastus Haindongo

Gabrielle (Abby) Harrison

Rachel Lowe

Fredrick Lutwama

Kerryn Moore

Roberta Prado

Juan Quintana

Ronald Ramirez

Gillian Stresman

Sofonias Tessema

Vanessa Tran

Delenasaw Yewhalaw

Elizabeth Adedire

Muhammed Afolabi

Lorna Chebon

Thierry Franchard

Majidah Hamid-Adiamoh

Hamidou Ilboudo

Said Jongo

Natasha Laban

Judith Mangeni

Nattika Nantachit

Eric Ndombi

Luicer Olubayo

Mariella Quispe

Ava Kristy Sy

Patrick Valere Tsouh Fokou

Hugo Valdivia

Gillian Eastwood

Geoffrey Gonzalez-Escobedo
Seokyoung Kang

Jessica McCaffery

Monica Nayakwadi Singer

Aaron Neal

Adam South

2014

Susan Adakun

Ben Andagalu

Elhadji $\mathrm{Ba}$

Herbert de Matos Guedes

Kebede Deribe

Bismarck Dinko

Fernanda Fortes de Araujo

Rubona Joshua

Elijah Juma

Jean Paul Katsuva

Stella Kepha

Evaristus Mbanefo

Seleshi Mekonnen

Dominic Mosha

Augusto Nhabomba

Rasaq Olaosebikan

Lynette Oyier

Mwiche Siame

Maggy Sikulu

Braulio Valencia

Alvaro Baeza Garcia

Elissa Butler

Suresh Maddur Ganesan

Frankline Magaki Onchiri

Patricia Pavlinac

Raul Saraiva

Suwanna Chaorattanakawee

Alex Eapen

Kimberly Fornace

Michael Hawkes

Danika Hill

Taweewun Hunsawong

Placide Mbala

Randy Mungwira

Sophie Namasopo

Jeremiah Ngondi

Repon Paul

Chandary Rang

Stephen Rulisa

Toolika Singh

Holger Unger

Rajesh Valanparambil

Tom Wingfield

\section{3}

Mary Auma

Geral Baldeviano

Awa Bineta Deme

Titus Divala

Adriana Echazu

Thanyalak Fansiri

Giovan Gomez
Maria Isabel Hoshi

Kofi Kwofie

Nancy Matowo

Clement Meseko

Agnes Nanyonjo

Jovvian Parakkal

Anuradha Rajamanickam

Wanlapa Roobsoong

Joan Simam

Isaac Ssewanyana

Chun Hai Fung

Nona Jiang

Melissa Nolan

Wesley Solomon

Lindsey Turnbull

Nana Wilson

Poom Adisakwattana

Bahita Ashenafi Assefa

Pebanne Atemba

Kodjo Ayi

Sarah-Blythe Ballard

Kayla Barnes

Douadi Benacer

Elizabeth Brickley

Georges Diatta

Clement Elanga N'Dille

Robyn Elphinstone

Mario Jiz

Stephen Kinara

Worachet Kuntawunginn

Belinda Morahan

Birgit Nikolay

David Pigott

Michael Smout

Hyacinthe Toe

Timothy William

Guang Xu

\section{2}

Ishag Adam

Kathryn Anderson

Ibrahima Baber

Paul Bangirana

Andrew Bouley

Natalie Bowman

Dibyadyuti Datta

Helen Gerns

Farhana Haque

Grace Kaguthi

James Kapisi

Samson Kiware

Pharath Lim

Martin Lukindu

Dickson Lwetoijera

Nelson Naranjo

Ousmane Ndiath

Winnie Okeyo

Prince Prabhu

Zhenpeng Qin 


\section{ANNUAL MEETING TRAVEL AWARD RECIPIENTS (cont.)}

Maurice Marcel Sandeu

Sanie Sesay

Om Prakash Singh

Tashi Tobgay

Rakiswendé Yerbanga

Min Zhang

Jennifer Armistead

Bridget Barber

Corey Campbell

Alexander Douglas

Jose Hagan

Emmanuel Hakizimana

Admirabilis Kalolella

Jingwen Lin

Moges Mekonnen

Louisa Messenger

Neida Mita-Mendoza

Elly Munde

Dyson Mwandama

Eugenie Poirot

Elizabeth Rogawski

Ebako Takem

Tiffany Weinkopff

Kee Thai Yeo

\section{1}

Sridhar Arumugam

Sania Ashraf

Ulysse Ateba Ngoa

Frederick Baliraine

Mariama Cherif

Kristina Clark

Maria del Pilar Crespo-Ortiz

Kamlesh Gidwani

Luis Gomez-Puerta

Quoc Cuong Hoang

Abel Kakuru

Elizabeth Keating

Albert Majwala

Emmie Mbale

Matthew McCarra

Hugues Clotaire Nana Djeunga

Jeanne Rini Poespoprodjo

Dumizulu Tembo

Nicholas Van Sickels

Emily Young

S. M. Niaz Arifin

Sanjiv Baxi

Dana Clifton

Tonya Colpitts

Noushin Emami

Constance Finney

Nahla Gadalla

Bich-Tram Huynh

Marwa Ibrahim

Tatiana Maria Lopera Mesa

Qianqian (Doreen) Miao

Nicolas Moiroux

Christopher Moxon
Eugene Oteng

Shahana Parveen

Christopher Peatey

Coraline Rigouin

Rungrat Saiyasombat

Naman Shah

Yeung Tutterrow

Linda Wammes

2010

Judith Anchang-kimbi

Tavis Anderson

Jennifer Chau

Kiprotich Chelimo

Carolina Cura

Emilie Dama

Dziedzom de Souza

James Kelley

Sammy Kiplagat

Drissa Konate

Monica Namayanja

My Phan

Andre Lin Pitaluga

Lincoln Timinao

Elizabeth Villasis

Jordan Zuspann

Ruth Ashton

Rene Condori

Jennifer Downs

Laura Erdman

Bernardo Franklin

Lauren Gong

Chris Keh

Alison Kuchta

Issa Lyimo

Ruth Nyakundi

Andre Lin Ouedraogo

Fred Piel

Karen Polson

Abdullah Sayeed

Dea Shahinas

Salvador Tarun

\section{9}

Yaw Afrane

Some Anyirekun Fabrice

Samuel Anyona

Albert Auguste

James Bangura

Jun Cao

Maria Dantur Juri

Iqbal Elyazar

Mofolusho Falade

Rafael Freitas

Najia Ghanchi

Lina Gutiérrez Builes

Md. Hossain

Adela Jiram

Basile Kamgang
Adama Keita

David Langoi

Tatjana Lima

Hamma Maiga

Swati Mandal

Dairo Marin

Alberto Mendoza

Cyrille Ndo

Quyen N. Nguyen

Abdisalan Noor

Michael Odera

Bilha Ogola

Lilian Ogonda

Emelda Okiro

Evans Okoth

Richard Omore

Robert Opoka

Renzo Perales

Wasiq Rawasia

Richard Ssekitoleko

Sivapong Sungpradit

Marco Tovar

Cristian Valencia

Nicolas Veland

Joanne Baker

Andrea Conroy

Jose Gaudier

Simon Glover

Vinay Gupta

Whitney Harrington

Rosalind Howes

Anam Javed

Regina Joice

Hae Ji Kang

Stephan Karl

Richard Maude

Atis Muehlenbachs

Panote Prapansilp

Melissa Riedesel

Amy Savage

Karlee Silver

Argon Steel

Esther Volper

Catherine Westbrook

Caroline Gitonga

John Keven

Siaka Konate

Daniel Minja

Bartholomew Nyangahu Ondigo

Daniel Onguru

Kayla Wolofsky

2008

Ambroise Ahouidi

Emmanuel Arinaitwe

Puji Asih

Patchanee Chootong

Astrid Cienfuegos

Cornell Collins Ouma 
ANNUAL MEETING TRAVEL AWARD RECIPIENTS (cont.)

\author{
Luc Djogbénou \\ Papa Drame \\ Kwadwo Frempong \\ Dionicia Gamboa \\ Bruno Ghersi \\ Alisa Junpee \\ Muhammed Khan \\ Joseph Koroma \\ Bhagyashree Manivannan (Uradey) \\ Erick Muok \\ Norah Mwebaza \\ Joaniter Nankabirwa \\ Sammuel Nsobya \\ Charles Obonyo \\ Pamela Orjuela-Sánchez \\ Surendra Kumar Prajapati \\ Anne Spichler \\ Iskra Tuero \\ Tom Were Wasike \\ Sheri Anderson \\ Maria Arevalo \\ April Bobenchik \\ Richelle Charles \\ Kelsey Deus \\ Anne Dickson \\ Brett Ellis \\ Christen Fornadel \\ Phillip George \\ Kathryn Griffiths \\ Aaron Harris \\ Yan $\mathrm{Hu}$ \\ Cynthia Khoo \\ Fiona Lovegrove \\ Robin Moudy \\ James Mutunga \\ Agnes Mwakingwe \\ Sarah Olson \\ Edsel Maurice Salvana \\ Maria de Jesus Trovoada \\ Matt Tucker
}

\section{7}

Mouhamadou Chouaibou

Rachaneeporn Jenwithisuk

Colince Kamdem

Hortance Manda

Flavia McBride

Arthur Mpimbaza

Daniel Olusola Ojurongbe

John Ongecha

Virornpun Sanprasert

Mariana Simoes

Peter Sumba

Jane Achan

Paige Adams

Christopher Barker

Ron Birnbaum

Paola Boggiatto

James Colborn
Francisco Diaz

Nancy Duah

Gretchen Ehrenkaufer

Autumn Girouard

Andrew Helmers

Andres Herrera

Nusrat Homaira

Emily Jentes

Marisa Madison

Frank Mannix

Charles McGee

Hugo Razuri

Win Surachetpong

Alexander Yaw Debrah

\section{6}

Tran Chau Nguyen

Rushina Cholera

Josue da Costa Lima

Judy Easterbrook

Joseph Fair

Darryl Falzarano

Yvette A. Girard

Moses R. Kamya

Ibne Karim M. Ali

Rebekah Kent

Mark Kuniholm

Daniella Martins

Kriti Mittal

Luciano Moreira

Kija Ng'habi

Denise Njama-Meya

Jonathan M. Sherman

Ratawan Ubalee

Tsin Wen Yeo

Karine Zevallos Villegas

\section{5}

Andrea Bertolotti-Ciarlet

Douglas Boettner

Juan Calix

Lon Chanthap

Tran Thuy Chau

Marina Chavchich

Julie Clennon

Hanan Helmy

Sulggi Lee

Khaled Mahmoud Abd Elaziz Saleh

Joao Mendes Wanderley

Sammy Njenga

Mike Osei-Atweneboana

Tasanee Panichakul

Narong Ponsa

Innocent Safeukui

Melanie Samuel

Hai-Wei Wu

2004

Catherine Bourguinat
Emmanuel Emukah

Krystal Evans

Vidal Felices

Guido Jordan

Renee Larocque

Kim Sung Lee

Jun Li

Emily Mann

Sean Murphy

Milagro Oviedo

Eddy Segura

Scott Shone

Hastings Suba

Katherine Swanson

Jessica Tarleton

Andrew Walsh

Adoke Yeka

Carol Zavaleta

2003

Sujittra Chaisavaneeyakorn

Emukah Chukwudirim

Alfred Cortes

Sophie Escutenaire

Anne Gasasira

Kari Gillenwater

Diego Andres Goyeneche

Patino

Gisely Hijar Guerra

Mayra Jimenez

Thomas Kariuki

Daniel Kyabayinze

Richard Reithinger

Jessica Ricaldi

Claudio Rocha

Moises Sihuincha

Sodiomon Sirima

2002

Ibrahim Abdel-Messih

Wallis Best-Plummer

Kimberly Brouwer

Maria Corena

Antoine Diatta

Fraction Dzinjalamala

Steven Gajda

Karen Haag

Mayra Jimenez

Renee Larocque

Ton That Long

Alemka Markotic

Rania Milleron

Fernando Monteiro

Victor Mwapasa

Salima Nathoo

Tien Huy Nguyen

Adriana Troyo

Wei-Kung Wang 


\section{ANNUAL MEETING TRAVEL AWARD RECIPIENTS (cont.)}

2001

Kai-Hsin Chang

Darja Duh

Heather Ferguson

Leticia Franco

Anne Gasasira

Iveth Jimenez

Sarah Keenihan

Thewarach Laha

Bill Messer

Raphael Oguariri

Cielo Pasay

Hakim Sendagire

Wondji Sinclair

Balbir Singh

Eduardo Villamor

Marcus Zachariah

Wenbao Zhang

\section{0}

Josephine Birungi

Jason Botten

Louis Casta

Randall DeJong

Meghna Desai

Eric Dumonteil

Rashika El Ridi

Lyndal Emerson

Elvia Esparza

Idle Farah

Jariyanart Gaywee

Mark Herb

Edward Kamau

Annie-Claude Labbe

Thanh Hoa Le

Yibayiri Sanogo

\section{9}

Aurimar Ayala-Lopez

Astrid Cardona

Remi Charrel

Mawlouth Diallo

Tedros Ghebreyesus

David Guiliano

SimonKariuki

Yuesheng Li

P'nLoke

Paula Mwinzi

Dylan Pillai

Elena Pollina

Pornpirnol Rongnoparut

Amadou Sall

Miwako Takano-Lee

Amelia Travassos da Rosa
1998

Robert Anderson

Jamaree Bhisutthibian

Adrian Billings

Olivier Garraud

Julia Gill

Pascale Leonard

Melissa Nevils

Nattiya Pimtanothai

Andrea Pomrehn

Janine Ramsey

Blanca Restrepo

Mario Santiago

Joanna Schaenman

Frederic Simard

Jean Tsao

Nathan Wolfe

1997

Gemiliano Aligui

Gabriel Bonilla

Lynn Cooper

Franka Des Vignes

Diana Karanja

Paul Levett

Kyle McKenna

Karkada Mohan

M. Angela Montesano

Caroline Othoro

Miroslav Petrovec

Windell Rivera

Lena Serghides

Joram Siangla

John Waitumbi

\section{6}

Oleg Alexejev

Norma Bautista-Lopez

Mausumi Bharadwaj

Edmund Browne

Marcelo Fantappie

Irina Gavrilovskaya

Luis Giraldo

Kim Halpin

Timothy Kram

C. Mazine

Kyeen Mesesan

Jaba Mukhopadhyay

M. Ramadan

Elaine Rodrigues

Ed Ryan

Slavia Ryder

Margarita Samudio

Jetsumon Sattabongkot
Sudha Vippagunta

Larisa Vredevoe

Dawit Wolday

Xiaoping Ye

1995

Gabriela Aviles

Dan Bausch

Wagner Dos Santos

Dao Duc

Khalid El-Shewy

I. Ghosh

Joan Gigstad

Lilia Gonzalez-Ceron

Adi Gundlapalli

Rashidul Haque

Diana Isaza

Veronica Jennings

Chris Leptak

Silvana Levis

Ivelina Metcheva

Ayub Ofulla

D. Oliveira

Boonyos Raengsakulrach

Sacha Sidjanski

C.N. Wamae

1994

Mohamed Ali

Denis Boulanger

Ana Maria Briggiler

Andrew Brittingham

Arturo Centurion

Sansanee Chaiyaroj

Alexandre da Silva

Davin Dillon

Kashinath Ghosh

Andrew Githeko

Said Ismail

Michael Lindsay

Rosalia Lira-Carmona

Lucy Lum

Peter McElroy

James Montoya-Lerma

Julie Moore

Alexander Rivkin

Inge Sutanto

L.A. Tchuem Tchuente

Bruno Travi

Jason Wooden 


\section{CERTIFICATE OF KNOWLEDGE IN CLINICAL TROPICAL MEDICINE AND TRAVELERS' HEALTH-CTropMed ${ }^{\circledR}$ PROGRAM}

\section{Recipients}

Daniel Adams

Adekola Adedeji

Ghassan Al Awar

Ragheed Al-Dulaimi

Wang Dingyuan Alvin

Ekta Bansal

Sapha Barkati

Rachel Bensman

Nathan Bertelsen

Divya Bhamidipati

Vijai Bhola

Cary Bjork

Amy Borden

Daniel Bourque

Baljeete Brar

Whitney Bryant

Helene Calvet

Lloyd Cassidy

Jessica Cataldi

Aileen Chang

Christine Chen

Justy Antony Chiramal

Samantha Chittick

Eva Clark

Sigrid Collier

Denrick Cooper

Nathanial Copeland

Katherine Crabtree

Juliana Da Silva

Kevin Dieckhaus

Abdelghani El Rafei

M. James Eliades

Shannon Findlay

Molly Fleece

Dianne Frankel

Ayako Wendy Fujita

Jessica Gaulter

Timothy Gobble

Laura Greisman

Daniel Guiles

Elizabeth Gulleen

Joel Hamm

Jessica Hane

Michael Heiland

Brett Hoffecker

Mary Holland

Rezhan Hussein

Jack Hutter

Saki Ikeda

Paul Janoian

Thomas John

Christie Joya

Pallavi Kamra

Anubhav Kanwar

John Paul Kelada

Aisha Khatib

Christine Kley

Parvathi Kumar

Varkha Lachman

Poppy Markwell

Martha Montgomery

Jennifer Masel

Lacey MenkinSmith
Mahesh Menon

Mary Micikas

Tara Ness

Samuel Oon Soo Ng

Thundon Ngamprasertchai

Andrew Olson

Taiwo Omole

Paul Opare-Addo

Susan Owens

Juan Perez Velazquez

Aleksandra Prochowska

Robert Pueringer

Bradley Randles

Arathi Rao

Pryanka Relan

Robert Rolfe

Joelle Rosser

Julia Rubin-Smith

Sima Saleh

Amanda Schnee

Timothy Smith

Nathan Stockamp

Joseph Sullivan

Hiroyuki Suzuki

Olympia Tachopoulou

Lokachet Tanasugarn

Charles Teh

Supatida Tengsupakul

Michelle Tubman

Chandler Villaverde

Benjamin Walz

Sara Westergaard

Walter Winders

Janewit Wongboonsin

Margaret Wood

Andrew Wu

Benjamin Wyler

\section{Recipients}

Mahsa Abassi

Lani Ackerman

Patrick Allen

Jay Allison

Anjoli Anand

Kristina Angelo

Prudence Barrett

Jennifer Beaudreau

Ronald Beaudreau

Dale C. Betterton

Jamie Boudreau

Danielle Brown

Mariel Bryden

Matthew Campbell

Wesley Campbell

Jeffrey Cho

Natalie Cobb

Roxana Cruz

James DellaValle

Timothy Depp

Malini DeSilva

Adriana Dhawan

David D'Souza

Michael Esan

Tracy Evans-Gilbert
Clayton Foster

Michael Gamble

Kathleen Gang

Christopher Gifford

Lisa Gilbert

Matthew Goers

Daniel Griffin

Matthew Haldeman

Crystal Hammer

Julie Hanson

David Harris

Bradley Hickey

Natasha Hochberg

Meghan Hofto

Peter Holmberg

W. Hunt

Claire Italiano

Michael Jaung

Jaimini Jodhani

Christopher Jones

Shanthi Kappagoda

Benjamin Katz

Mandip KC

Adam Kley

Alexia Knapp

Michael Koren

Michelle LaBrunda

Andrea Lack

Stephanie Lauden

Kellene Lenz

Andrew Letizia

Daniela Lobo

Jason Lohr

Michael Marriott

Donna McGregor

Brian Medernach

Ana-Claire Meyer

Bayan Missaghi

Joshua Mularella

Lindsay Murphy

Philip Murray

Eric Nau

Jill Neely

Chienyenwa Nwachuku-

Winful

Elise O'Connell

Meagan Pate

Carolina Perry

Jonathan Peters

Hope Pogemiller

Vivek Ramarathnam

Mauricio Rebolledo

Medranda Heather Rivasplata

Candace Rypien

Sapna Sadarangani

Rebecca Sainato

Makoto Saito

Kacie Saulters

Martin Schmidt

Michael Smit

Linnea Smith

Melissa Sutton

Andrzej Szefler

Wilawan Thipmontree
Alexandra Vinograd

Jill Weatherhead

Soe Win

Jennifer Wong

Rebecca Zadroga

Anteneh Zewde

2014 Recipients

Kathryn Anderson

Ana Arauz

David Ayer

Meagan Barry

Luther Bartelt

Katherine Billingsley

Tim Bongartz

Christine Borghi-Cavallaro

David Brieff

Calla Brown

Benjamin Bryden

Darilyn Campbell-Falck

Rebecca Chancey

Lisa Chastant

Roshni Daver

Bethany DeBoer

Britta Denman

Devika Dixit

Jon Dyal

Karen Ekernas

Ryan Fabrizius

Sheiphali Gandhi

Cameron Gongwer

Maria Guevara

Daniele Gusland

Tracey Haas

Lesca Hadley

Kamal Hamed

David Horne

Benjamin Huntley

Rosanna laniro

Alexander Kay

Leah Kern

Roy Kim

Rachael Krob

Karl Kronmann

Eric Lee

Christopher Lindshield

Rebecca Lyle

Salahudin Maalim

Rajat Madan

Stephanie Malherbe

Suma Manjunath

Luis Marcos

Megan McKinnon

William Meeker

Llewellyn Mensah

Sandy Mudge

Carolyn Newman

Naranjo Theresa Nguyen

Obinna Nnedu

Jody Olson

Padmasayee Papineni

Jaime Pardo

Lorine Pelly

Jeffrey Percak 


\section{CERTIFICATE OF KNOWLEDGE IN CLINICAL TROPICAL MEDICINE AND TRAVELERS' HEALTH-CTropMed ${ }^{\circledR}$ PROGRAM (cont.)}

Stefan Pomrenke

Andrew Posey

Jakrapun Pupaibool

Anupama Raghuram

Nipunie Rajapakse

William Ramsey

Manoj Ray

Lauren Richey

Marie Rubby

Roger Reinaldo

Rosas Carlo

Rossi Richard

Ruck Marcus

Salmen Aaron

Scott Siavash Shahbodaghi

Anita Sircar

Kristina St. Clair

Aubrey Stimola-Ryan

Anup Subedee

Ramona Sunderwirth

Leigh Sweet

Rachel Talavlikar

Milada Tavodova

Michael Taylor

Susan Tector

Elizabeth Thielen

Joshua Thomas

Nicholas Van Sickels

Andrea Van Wyk

Peter Veldkamp

Indumathi Venkatachalam

Karolyn Wanat

Amber Wobbekind

Edward Xie

Karen Yates

Anthony Zimmerman

2012 Recipients

Sharjeel Ahmad

Brandon Allport

Margot Anderson

Nasikarn Angkasekwinai

Nathan Bahr

Sarah-Blythe Ballard

Brenda Barnhart McGuire

Theresa Barton

Jason Blaylock

Matthew Caddell

Steven Cashen

Cesar Augusto

Castro de Barros

Elizabeth Clarke

Keri Cohn

Douglas Collins

Glen Drobot

Dan Dulek

Robert Ferris

DeAnna Friedman

Brandi Gary

John Gibson

Rafik Hanna

Gretchen Heinrichs

Jeffrey Henney

Elisabeth Hesse

Andrea Hull
Camille Introcaso

Neville Jadeja

Lucas Johnson

Ayesha Kadir

Yasuyuki Kato

Michael Kavanaugh

Sarah Kesler

Karl Kirby

Anjali Kunz

Daniel Lattanzi E

dward Leitz

Leyi Lin

Jill Linse

Raymond Lyrene

Pasri Maharom

Sarah Mandigo

Ashley Maranich

Yasutaka Mizuno

Vasant Nagvekar

Kristin O'Dell

Anil Panackal

Kristopher Paolino

Elizabeth Petersen

Timothy Ruttan

Kimberly Schoonover

Christian Seefelder

Megan Shaughnessy

Judith Steyer

Mark Swancutt

Ashwini Tayade

Andrea Tenner

Nicole Thomas

Drake Tilley

Harrys Torres

James VanVooren

Stephen Vaughan

Katherine Venable

George Viola

Terry Wuerz

Kosuke Yasukawa

Rudy Zimmer

\section{Recipients}

Jan Agosti

Julie Ake

Svenja Albrecht

Rahul Anand

Suzanne Atteberry

Mariam Aziz

William Bevins

Brian Blackburn

Natalie Bridger

Benjamin Bristow

Ulrike Buchwald

Amaya Bustinduy

Seema Chandra

Gordon Christensen

Kevin Clarke

Laura Cooley

Peter J. De Vries

Gregory Deye

Michael Ellis

Joseph Etienne

Jessica Fairley

Eileen Farnon
Ahmed Farooq

Norman Farr

Jacqueline Firth

Eric Garges

Jorge Garib

Cameron Gifford

Curtis Gongwer

Jonathan Grennan

Dubert Guerrero

Jeffrey Hall

Joshua Hartzell

Sarah Hoehnen

Julia Hutter

Shamim Islam

Mark Johnson

Matthew Jones

Ubonvan Jongwutiwes

Stephen Jost

Michael Khoury

Kristi Klee

Kristina Krohn

Paul Larson

Sarah Lee

David Lohr

Rogelio Lopez-Velez

Joel Massey

Jacqueline Michaud

Vicki Penney

Benjamin Phelps

Marc Robinson

Rodrigo Romulo

Daniel Schnobrich

Charles Schubert

Michelle Science

Phillip Scott

Thomas Snickenberger

Venkat Srinivasan

Dorairajan Suresh

Kumar Carey Terry

Damanpreet Ubhi

Michael Virata

Paige Waterman

Brian Yablon

Mohamed Yassin

1995-2008 Recipients

Neva Abbott

Elfatih Abter

Samer Abufadil

Shadab Ahmed

Azeem Ahsan

Abdulkader Al Rezqi

Ghassan Al-Awar

Tarik Al-Azraqi

David Alcid

Sadia Ali

Donald Allegra

James Allen

William Allen

William Alto

Fernando Alvarado

Carlos Alvarez

Hassan Amjad

Natasha Anandaraja

Steven Andersen
Susan Anderson

Benjamin Andrews

Anne Anglim

Vernon Ansdell

Deepak Ariga

Carlos Aristeguieta

Robert Armstrong

Mark Arness

Naomi Aronson

Ashoni Arora

Julio Arroyo

Ramin Asgary

Tobey Audcent

Arash Babaoff

Lindsey Baden

Steven Baker

Iqbal Bal

Michael Ballard

Anna Banerji

Shehla Baqi

Elizabeth Barnett

V. Theodore Barnett

Michael Barnish

Michele Barry

Robert Barthel

Alex Baskous

Daniel Bausch

Andrew Bazemore

Donald Beggs

Kiran Belani

Joseph Bell

Jean-Luc Benoit

Stephen Berger

Vladimir Berthaud

Larry Bethoney

Deborah Bettels

Lee Beyer

Frank Bia

Kendall Billick

Joseph Bingham

Marvin Bittner

David Blazes

Samia Boctor

Diane Boteler

Alain Bouckenooghe

David Boulware

Terra Bowles

Russell Bradford

David Brandt

Darin Brannan

Lo Ranee Braun

Gerald Brennock

Holly Brodzinski

Jason Brophy

Arthur Brown

Joel Brown

Richard Brown

Joe Bryan

Don Buckley

Yen-Giang Bui

Timothy Burgess

Heather Burnett

Mark Burnett

Joseph Byrnes

Claude Cadoux 


\section{CERTIFICATE OF KNOWLEDGE IN CLINICAL TROPICAL MEDICINE AND TRAVELERS' HEALTH-CTropMed ${ }^{\circledR}$ PROGRAM (cont.)}

John Campagna

Daniel Caplivski

G. Alexander Carden

I. Dale Carroll

Romanee Chaiwarith

Jeff Chapman

William Chapman

Paul Charlebois

Lin Chen

Imtiaz Choudhary

Sarfraz Choudhary

Emilie Chow

Greg Christensen

John Christenson

Vishnu Chundi

Preston Church

Theodore Cieslak

Elizabeth Clark

Marvin Clark

F. David Clifford

Barnett Cline

Jeffrey Cloud

Allison Clough

Matthew Coatsworth

Jonathan Cohn

Mireille Coles

Kenneth Colina

JoAnne Collinge

Maria Compte

Byron Conner

Walter Connor

Timothy Cook

Timothy Cooper

Nicholas Corey

Paula Corrigan

Philip Coyne

John Crane

E. Patrick Creehan

Maryanne Crockett

Tonya Crook

Nancy Crum

James Crutcher

Johanna Daily

Harry Davis

Richard Dawood

Mohamud Daya

John De Pasquale

Robert DeFraites

Pierre Dejace

Anne-Marie Demers

Arlene Dent

Stan Deresinski

Jaime Deseda

Ronald Devine

Vinod Dhawan

Lew Dick

David Diemert

Daniela Dilorio

Ross Donaldson

Mary Donnelly

Deirdre Duffy

Dana Duncan

Terry Dwelle

Kenneth Earhart

A. Mark Edwards
Edward Eitzen

Okechukwu Ekenna

Randall Ellis

Robert Elwood

Wesley Emmons

Carol Encarnacion

Arne Eskesen

Ana Maria Espinoza

Dennis Faix

Nagib Fares

R. Wesley Farr

Getachew Feleke

Jovita Fernandez

Michael Fincher

Philip Fischer

Evelyn Fisher

Walter Fister

Susannah Ford

Arthur Forni

John Foster

Fabio Foti

LeAnne Fox

Carlos Franco-Paredes

Leah Frazier

David Freedman

Daniel Freilich

J. Gordon Frierson

Mary Gabriel

Robert Gasser

Paula Gettys

Neil Gibson

Mary Glass

Stephen Gluckman

Ruth Goehle

William Goellner

Jesus Gonzalez-Moreno

Ram Gopalakrishnan

Hector Gorbea

Kara Goss

Philip Gould

Michael Grieco

Jeffrey Griffiths

Matthew Guarino

Fernando Guerena

Richard Guerrant

Mary Ellen Guroy

Sandra Gustin

Frank Gutmann

Christiane Hadi

Gary Haffner

Stefan Hagmann

Rana Hajjeh

Braden Hale

De Von Hale

Jennifer Halverson

Davidson Hamer

John Hammer

James Hansen

Scott Harper

Cathleen Harris

Scott Harris

Jonathan Harte

Michael Hawkes

Konrad Hayashi

Debbie Heit
Brett Hendel-Paterson

Todd Henrikson

Christy Henry

Timothy Herrick

Barbara Herwaldt

Patrick Hickey

Alicia I. Hidron

John Hii

David Hill

Julie Hoffman

Stephen Hoffman

Paul Holtom

Michael Holzer

Jennifer Hong

Duane Hospenthal

Eric Houpt

Randolph Hurley

Rocio Hurtado

Ahmed Hussein

Jeffrey Jackson

Frederique Jacquerioz

Andrew Jamieson

John Janikowski

Thomas Jensen

Nishith Jobanputra

Christine Johns

Betty Johnson

Donald Johnson

Edward Johnson

Leonard Johnson

Maxwell Johnson

Stephen Johnson

Jeffrey Jones

M. Patricia Joyce

Gregory Juckett

Kendrick Kahler

Shiva Kalidindi

Alexander Kallen

William Kammerer

Bryan Kapella

Michele Karim

Christopher Karp

John Kasukonis

Lisa Keep

Alan Scott Kellermann

Timothy Kelton

Richard Kenney

Henry Kerandi

Thomas Kerkering

Ali Khan

Riad Khatib

Charles King

Timothy Kirchler

Beth Kirkpatrick

Lynn Kitchen

Amy Klion

Colleen Kniffin

Joseph Kolars

Hoonmo Koo

Mark Kortepeter

Philip Korthuis

Carrie Kovarik

Victor Kovner

Phyllis Kozarsky

Mark Krautheim
Steven Krotzer

Tim Kuberski

Sharon Kuhn

Susan Kuhn

Walter (Ted)Kuhn

Sampath Kumar

Eileen Kummant

Farrukh Kureishy

Awewura Kwara

Mark Lacy

John Lammie

Michael Lange

Larry Laughlin

Chinh Trung Le

Richard Leach

Timothy Leach

John Leake

Erica Leazenby

Elizabeth Ledbetter

Edith Lederman

Lawrence Lee

Emil Lesho

Ludwig Lettau

Francois Lette

Ine Leus

Jill Levy

Drew Lewis

Daniel Libraty

Poh-Liam Lim

Jessica Lin

David Lintz

Jay Litchfield

Joanne Liu

Carson Lo

Charles Longer

Janice Louie

Daniel Lucey

Aric Ludwig

Thomas Luke

Kevin Lunney

Julia Lynch

Michael Lynch

Jonathan MacClements

Susan MacDonald

Sheila Mackell

Alan Magill

James Maguire

Jason Maguire

Charles Mahan

Siddhartha Mahanty

Sumit Majumder

Mark Malakooti

John Malone

Joseph Malone

James Mancuso

Venkat Mani

Doreen Mar

Leonard Marcus

William Markle

Lucila Marquez

Muriel Marshall

Pierre Martel

Gregory Martin

Harold Martin

James Martin 


\section{CERTIFICATE OF KNOWLEDGE IN CLINICAL TROPICAL MEDICINE AND TRAVELERS' HEALTH-CTropMed ${ }^{\circledR}$ PROGRAM (cont.)}

Carl Mason

Glenn Mathisen

David Mathison

Kunjana Mavunda

Sherman McCall

Martin McCann

Anne McCarthy

Karen McClean

Lesley McGalliard

Kelly McKee

Carol McLaughlin

Susan McLellan

Michael McMahon

David McNeeley

Ameya Medhekar

Peter Melby

Paul Mertens

Rebecca Meyerson

Larry Miller

Robert Miller

Jacob Minor

Ayesha Mirza

Yazdan Mirzanejad

Edgar Molina

Martin Montes

Christopher Moore

Thomas Moore

John Moran

Shaun Morris

Robert Mott

Victor Mulanovich

Stephen Munday

Gerald Murphy

John Murphy

Timothy Myrick

Jean Nachega

Yoshitaka Nakagawa

Leenhapong Navaravong

Franklin Neva

Patrick Ney

Ank Nijhawan

Richard Niska

Scott Norton

Thomas Nutman

Chidi Nwizu

Sean O'Donnell

Richard Oberhelman

Christophe Ohl

Patrick Olson

Mario Onagan

Winnie Ooi

Steven Oscherwitz

Johanna Osorio

Barry Pakes

Pia Pannaraj

Claire Panosian

Scott Paparello

Mark Paris

Douglas Parkin

Dean Paschal

Prafullkumar Patel
Jan Patterson

Julie Pavlin

Richard Pearson

M. Sayonara Perez

Mato Jeffrey Pernica

Mireille Plamondon

Christopher Plowe

John Podgore

Herbert Prawius

Todd Price

Ronald Primas

Ronald Pust

Gregory Raczniak

Shobita Rajagopalan

Latha Rajan

Diana Ramirez-Baron

Nigel Raymond

David Reifsnyder

Edward Rensimer

Steven Reynolds

Thomas Richie

Mark Riddle

Terri Rock

David Roesel

Matthew Rollosson

Martha (Marty) Roper

Jon Rosenblatt

Meghan Rothenberger

Debra Rowse

Omid Rowshan

Larry Rumans

Edward Ryan

Katie Ryan

Maheen Saeed

Rolando Saenz

Edsel Salvana

Priya Sampathkumar

Benjamin Samuel

John Sanders

Michael Sands

Christopher Sanford

Scott Santibanez

Arif Sarwari

Michael Sauri

Susan Saxe

Lynora Saxinger

Andrew Schechtman

Gottfried Schmer

Friedel Schneider

Glenn Schnepf

Sandra Schumacher

Mary-Louise Scully

Stephen Sears

Nyo Sein

Uzi Selcer

Jose Serpa-Alvarez

Trueman Sharp

Michael Shertz

Margarita Silio

Dawd Siraj

Joseph Sliman
Michael Sluss

Raymond Smego

Clyde Smith

Darvin Smith

Mark Smith

Melodie Smith

Bonnie Smoak

Keith So

Georgia Sotiropoulos

Paul Southern

David Spees

Corydon Sperry

Hans Spiegel

Alan Spira

Frank Stackhouse

Sarah Staedke

William Stauffer

Bruce Steffes

Madlyn Stein

Ellen Steinberg

Stevenson

Theodore Steiner

Robert Stewart

Mark Stinson

Steven Stocks

Oystein Strand

Judy Streit

Lara Strick

Alec Style

Andrea Summer

Deena Sutter

Robert Swanson

Stephen Swanson

Thor Swanson

Paul Swoboda

Elizabeth Talbot

Paul Tambyah

Cindy Tamminga

Sybil Tasker

Kevin Taylor

Nathan Teague

Ildefonso Tellez

Tom Thacher

Donald Thea

Michael Thigpen

Stephen Thomas

Donald Thompson

Matthew Thompson

Robert Tingley

Ellis Tobin

Michael Tolle

Kay Tomashek

Jason Tompkins

Stephen Toovey

John Toso

Indi Trehan

David Tribble

Sally Trippel

Andrew Trofa

April Truett

Anthony Tvaryanas
O Ugwu

William Umhau

Gregory Utz

David Van Dyke

John Vande Waa

Mary Alice Vanderkooi

Sabah Varoqua

Cuddalore Vasudevan

Sten Vermund

Merceditas Villanueva

Brant Viner

Joseph Vinetz

Christopher Vinnard

Steven Virant

Roopa Viraraghavan

Abinash Virk

Cecily Wait

Patricia Walker

Arthur Wallace

Mark Wallace

Douglas Walsh

Karen Wang

Shu-Hua Wang

Cirle Warren

Glenn Wasserman

Julia Weeks

Gary Weil

Peter Weina

Eric Weiss

Peter Weiss

Peter Weller

A. Clinton White

Andrew White

Gordon Whitney

Peter Wiest

William Willitts

Mary Wilson

Mary Wilson

Patrick Wilson

Dana Witmer

Darrell Witt

David Witt

Murray Wittner

Martin Wolfe

Patricia Woodall

Kara Wools-Kaloustian

Glenn Wortmann

Andrew Wright

Paul Wright

Seth Wright

Randolph Wykoff

Richard Ybarra

Philip Yeon

Irina Yermilov

Cedric Yoshimoto

Jean Young

A.J. Yund

Kenneth Zangwill

Brian Zanoni

Michael Zapor 
ASTMH-Approved Diploma Courses in Clinical Tropical Medicine

Diploma in Tropical Medicine

Baylor College of Medicine

Diploma in Tropical Medicine

Baylor International Pediatric AIDS Initiative

Diploma in Tropical Medicine

Bernhard Nocht Institute

Gorgas Memorial Institute of Tropical and Preventive Medicine

Diploma in Tropical Medicine and Public Health

Charité University Medicine Berlin, Humboldt University Free University Berlin

Graduate Certificate in Tropical Medicine

John A. Burns School of Medicine at the University of Hawaii, Mãnoa

Summer Institute in Tropical Medicine and Public Health Johns Hopkins Bloomberg School of Public Health

Diploma in Tropical Medicine \& Hygiene

Liverpool School of Tropical Medicine

Diploma in Tropical Medicine \& Hygiene

London School of Hygiene and Tropical Medicine

East African Diploma in Tropical Medicine and Hygiene London School of Hygiene \& Tropical Medicine
Graduate Diploma in Tropical Medicine and Hygiene

Mahidol University

Graduate School of Tropical Medicine and Global Health (TMGH)

Nagasaki University

Postgraduate Course in Tropical Medicine and International Health

Prince Leopold Institute of Tropical Medicine

Diploma Course in Clinical Tropical Medicine and Travelers' Health

Tulane University

Training in Tropical Medicine and Travelers' Health Uniformed Services University of the Health Sciences

University of Minnesota Global Health Course University of Minnesota/Centers for Disease Control and Prevention

Intensive Course in Tropical and Travel Medicine University of Texas Medical Branch at Galveston

\section{Tropical Medicine and Global Health}

University of Virginia Health System

Clinical Tropical Medicine and Travelers' Health

West Virginia University

\section{ANNUAL MEETING HISTORY}

\begin{tabular}{|c|c|c|c|}
\hline $\begin{array}{l}2019 \\
\text { National Harbor, Maryland }\end{array}$ & $\begin{array}{l}2009 \\
\text { Washington, DC }\end{array}$ & $\begin{array}{l}1999 \\
\text { Washington, DC }\end{array}$ & $\begin{array}{l}1989 \\
\text { Honolulu, Hawaii }\end{array}$ \\
\hline $\begin{array}{l}2018 \\
\text { New Orleans, Louisiana }\end{array}$ & $\begin{array}{l}2008 \\
\text { New Orleans, Louisiana }\end{array}$ & $\begin{array}{l}1998 \\
\text { Cancelled-San Juan, Puerto } \\
\text { Rico (Hurricane Georges) }\end{array}$ & $\begin{array}{l}1988 \\
\text { Washington, DC }\end{array}$ \\
\hline $\begin{array}{l}2017 \\
\text { Baltimore, Maryland }\end{array}$ & $\begin{array}{l}2007 \\
\text { Philadelphia, Pennsylvania }\end{array}$ & $\begin{array}{l}1997 \\
\text { Orlando, Florida }\end{array}$ & $\begin{array}{l}1987 \\
\text { Los Angeles, California }\end{array}$ \\
\hline $\begin{array}{l}2016 \\
\text { Atlanta, Georgia }\end{array}$ & $\begin{array}{l}2006 \\
\text { Atlanta, Georgia }\end{array}$ & 1996 & $\begin{array}{l}1985 \\
\text { Miami, Florida }\end{array}$ \\
\hline $\begin{array}{l}2015 \\
\text { Philadelphia, Pennsylvania }\end{array}$ & $\begin{array}{l}2005 \\
\text { Washington, DC }\end{array}$ & $\begin{array}{l}\text { Baltimore, Maryland } \\
1995\end{array}$ & $\begin{array}{l}1984 \\
\text { Baltimore, Maryland }\end{array}$ \\
\hline $\begin{array}{l}2014 \\
\text { New Orleans, Louisiana }\end{array}$ & $\begin{array}{l}2004 \\
\text { Miami, Florida }\end{array}$ & $\begin{array}{l}\text { San Antonio, Texas } \\
\mathbf{1 9 9 4} \\
\text { Cincinnati, Ohio }\end{array}$ & $\begin{array}{l}1982 \\
\text { Cleveland, Ohio }\end{array}$ \\
\hline $\begin{array}{l}2013 \\
\text { Washington, DC }\end{array}$ & $\begin{array}{l}2003 \\
\text { Philadelphia, Pennsylvania }\end{array}$ & 1993 & $\begin{array}{l}1981 \\
\text { San Juan, Puerto Rico }\end{array}$ \\
\hline $\begin{array}{l}2012 \\
\text { Atlanta, Georgia }\end{array}$ & $\begin{array}{l}2002 \\
\text { Denver, Colorado }\end{array}$ & $\begin{array}{l}1992 \\
\text { Seattle, Washington }\end{array}$ & $\begin{array}{l}1980 \\
\text { Atlanta, Georgia }\end{array}$ \\
\hline $\begin{array}{l}2011 \\
\text { Philadelphia, Pennsylvania }\end{array}$ & $\begin{array}{l}2001 \\
\text { Atlanta, Georgia }\end{array}$ & 1991 & $\begin{array}{l}1979 \\
\text { Tucson, Arizona }\end{array}$ \\
\hline $\begin{array}{l}2010 \\
\text { Atlanta, Georgia }\end{array}$ & $\begin{array}{l}2000 \\
\text { Houston, Texas }\end{array}$ & $\begin{array}{l}1990 \\
\text { New Orleans, Louisiana }\end{array}$ & $\begin{array}{l}1978 \\
\text { Chicago, Illinois }\end{array}$ \\
\hline
\end{tabular}


1977

Denver, Colorado

1976

Philadelphia, Pennsylvania

1975

New Orleans, Louisiana

1974

Honolulu, Hawaii

1973

Houston, Texas

1972

Miami, Florida

1971

Boston, Massachusetts

1970

San Francisco, California

1969

Washington, DC

1968

Atlanta, Georgia

1967

Philadelphia, Pennsylvania

1966

San Juan, Puerto Rico

1965

New Orleans, Louisiana

1964

New York, New York

1963

Chicago, Illinois

1962

Atlanta, Georgia

1961

Washington, DC

1960

Los Angeles, California
1959

Indianapolis, Indiana

1958

Miami, Florida

1957

Philadelphia, Pennsylvania

1956

New Orleans, Louisiana

1955

Boston, Massachusetts

1954

Memphis, Tennessee

1953

Louisville, Kentucky

1952

Galveston, Texas

1951

Chicago, Illinois

1950

Savannah, Georgia

1949

Memphis, Tennessee

1947

Atlanta, Georgia

1944

St. Louis, Missouri

1943

Cincinnati, Ohio

1942

Richmond, Virginia

1941

St. Louis, Missouri

1940

Louisville, Kentucky

1939

Memphis, Tennessee
1938

Oklahoma City, Oklahoma 1920

1937

New Orleans, Louisiana

1936

Baltimore, Maryland

1935

St. Louis, Missouri

1934

San Antonio, Texas

1933

Richmond, Virginia

1932

Birmingham, Alabama

1931

New Orleans, Louisiana

1930

Cleveland, Ohio

1929

Miami, Florida

1928

Washington, DC

1927

Boston, Massachusetts

1926

Washington, DC

1925

Washington, DC

1924

Chicago, Illinois

1923

San Francisco, California

1922

Washington, DC

1921

Hot Springs, Arkansas
New Orleans, Louisiana

1919

Atlantic City, New Jersey

1918

Cancelled (Influenza)

1917

New York, New York

1916

Washington, DC

1915

San Francisco, California

1914

Boston, Massachusetts

1913

Washington, DC

1912

Atlantic City, New Jersey

1911

New Orleans, Louisiana

1910

St. Louis, Missouri

1909

Washington, DC

1908

Baltimore, Maryland

1907

New York, New York

1906

Philadelphia, Pennsylvania

1905

Philadelphia, Pennsylvania

1904

Philadelphia, Pennsylvania

\section{Named Lectures}

\section{COMMEMORATIVE FUND LECTURE-}

This lecture is presented annually by an invited senior researcher resident in the tropics.

2019

Moses R. Kamya

Makerere University

2018

Kelly Chibale

University of Cape Town
2017

Jane Cordosa

Sentinex Therapeutics

\section{6}

Zulfiqar Bhutta

Hospital for Sick Children and Aga Khan University
2015

Abdoulaye Djimdé

University of Science, Techniques and Technology of Bamako

2014

Eusebio Macete

Manhica Health Research Center 


\section{COMMEMORATIVE FUND LECTURE (cont.)}

2013

Nick Day

Mahidol-Oxford Tropical Medicine

Research Unit (MORU)

2012

John Gyapong

University of Ghana

\section{1}

Mirta Roses Periago

Pan-American Health Organization

\section{0}

Alejandro Cravioto

International Centre for Diarrhoeal

Disease Research, Bangladesh

\section{9}

Fred Binka

University of Ghana

\section{8}

Carlos Morel

Oswaldo Cruz Foundation (FIOCRUZ)
2007

Awa Coll-Sack

Roll Back Malaria Partnership

2006

Sambo Sow

Center for Vaccine Development-Mali

2005

Oyewale Tomori

Redeemer's University

2004

Eduardo Gotuzzo

IMT 'Alexander Von Humboldtt'

2003

Rashidul Haque

International Centre for Health and

Population Research

2002

Solomon Benatar

University of Cape Town

2001

Francis Nkrumah
Noguchi Memorial Institute for Medical Research

\section{0}

Socrates Herrera

Instituto de Immunologia Del Valle Cali

1999

R. Swanepoel

National Institute of Virology

1998

Ayoade M.J. Oduola (Invited)

University of Ibadan

1997

A. de Queiroz Sousa

Secretary of Health

1995

C.A. de Quadros

Pan American Health Organization

\section{CHARLES FRANKLIN CRAIG LECTURE-}

This lecture is presented in alternate years at the ASTMH Annual Meeting by a distinguished worker in the field of tropical medicine.

2018

Beth Kirkpatrick

University of Vermont College of

Medicine

\section{6}

Albert Icksang Ko

Yale School of Public Health

2014

Terrie Taylor

Michigan State University

\section{2}

Michael Alpers

Curtin University, Australia

2010

Robert Gilman

Johns Hopkins University School of Public Health
2008

Robert Swanepoel

National Institute for Communicable Diseases

2006

David Molyneux

Liverpool School of Tropical Medicine

2004

David Walker

University of Texas Medical Branch

2002

Barry J. Beaty

Colorado State University

1994

Michael Osterholm

Minnesota Department of Health
1992

Hon. D.A. Henderson

Office of Science and Technology Policy

1990

Thomas P.Monath

USAMRIID

1988

Duane Gubler

Centers for Disease Control

1986

Franklin Neva

National Institutes of Health 


\section{VINCENZO MARCOLONGO MEMORIAL LECTURE- This lecture is presented annually at the ASTMH Annual Meeting as part of the ACCTMTH meeting.}

\section{9}

Robert Handby

Australian Red Cross, Australia

The Provision of Safe Water in

Complex Environments

\section{8}

David Christiani

Harvard Medical School and Harvard

School of Public Health Boston, MA

Environmental Disease in Tropical

Regions

\section{7}

Claudio F. Lanata

Nutritional Research Institute,

Lima Peru

Vibrio Cholera: Lessons from Haiti and

Its Pending Research Agenda

\section{6}

Saliyapura

Sri Lanka

Leptospirosis in the Tropics: The

Diagnostic Challenge Suneth Buddhika

Agampodi

\section{5}

David A. Warrell

Oxford, United Kingdom

The Clinical Fascination of Snake-Bite

World Wide

\section{4}

Carlos Seas

Lima, Peru

Paraccocidiodomycosis: A Neglected

Mycosis of Latin America

\section{3}

Edgar Carvalho Salvador

Brazil

Management of Tegumentary

Leishmaniasis: Lessons from Studies on

Pathogenesis of Leishmania braziliensis infection"

\section{2}

Buddha Basnyat

Nepal International Clinic Kathmandu, Nepal

"Gall Bladder Carriage of Salmonella: A Case of Mistaken Identity"

\section{1}

Fredrick Sawe

Kenya Medical Research Institute/

Walter Reed Project Kericho, Kenya

"Prevention of Mother-to-Child

Transmission of HIV Infection and HIV

Free Survival:

Perspectives from Resource-Limited Settings"

\section{0}

Anita Zaidi

Aga Khan University Karachi, Pakistan
"Neonatal Infections: A Global

Perspective"

\section{9}

David A.J. Moore

Imperial College London and

Universidad Peruana Lima, Peru

"Paradigm Shifts in Tuberculosis Drug

Susceptibility Testing: New Dos and Donts"

\section{8}

Raul Isturiz Hospital Privado

Centro Medico de Caracas Caracas,

Venezuela

"Understanding Neurocysticercosis:

Advances in the Last 50 Years"

\section{7}

Christian Burri

Swiss Tropical Institute Basel, Switzerland

"Human African Trypanosomiasis: A

Neglected Disease with Low Prevalence But High Impact"

\section{6}

Kevin Marsh

KEMRI-Wellcome Trust Collaborative

Research Programme Kilifi, Kenya

"Severe Malaria: A Moving Target?"

\section{5}

"Cystic Echinococcosis: To Treat Or Not

To Treat?" Enrico Brunetti

University of Pavia Pavia, Italy

2004

Jacques Pepin

University of Sherbrooke Quebec, QC, Canada

"Human African Trypanosomiasis: The Past Explains the Present and Is Key to the Future"

\section{3}

Tom Solomon

University of Liverpool Liverpool, United Kingdom

"Japanese Encephalitis-West Nile's Ugly

Sister"

\section{2}

David Dance

Public Health Laboratory Plymouth, United Kingdom

"Melioidosis: The Peril in the Paddy Fields"

\section{1}

Edward Zijlstra

College of Medicine Blantyre, Malawi

"Visceral Leishmaniasis in Sudan: New Insights from the Past Decade"

\section{0}

Solly Faine

Monash University Melbourne, Australia
"Leptospirosis, the Hide-and-Seek Disease"

1999

Eli Schwartz

Tel Aviv University Israel

"Malaria Prophylaxis: A New Approach"

1998

Annual Meeting Cancelled due to Hurricane Georges

1997

Michael Roggendorf

Institute of Virology, University of Essen Germany

"Clinical Features and Epidemiology of Tick Borne Encephalitis in Central and Eastern Europe"

1996

Jean Pape

Cornell University Haiti

"An Infectious Disease Specialist in Haiti: AIDS, Diarrhea, Typhoid Fever and Civil Unrest"

1995

J.H. Ellner

Case Western Reserve University

Cleveland, $\mathrm{OH}$

"Tuberculosis: Developments in

Epidemiology, Diagnosis, Treatment and Prevention"

1994

Nicholas White

Hospital of Tropical Diseases, Mahidol

University Bangkok, Thailand

"Management of Severe and

Complicated Malaria"

\section{3}

Thiravat Hemachudha

Chulalongkorn University Bangkok, Thailand

"Human Rabies: Clinical Features, Pathogenesis and Potential Treatment"

1992

Anastacio de Queiroz Sousa Federal University of Ceara Fortaleza, Brazil

"HIV and Opportunistic Infections in Northeastern Brazil"

\section{1}

Anthony Bryceson

London School of Hygiene and Tropical

Medicine London, England

"Tropical Dermatology"

\section{0}

Reeder

Honolulu, HI

"Radiological Aspects of Tropical and

Parasitic Disease" 
FRED L. SOPER LECTURE-

This lecture is presented in alternate years at the ASTMH Annual Meeting by a distinguished scientist involved in studies related to environmental control and preventive medicine in the tropics.

$\begin{array}{lll}\mathbf{2 0 1 9} & \mathbf{2 0 0 9} & \mathbf{1 9 9 6} \\ \begin{array}{l}\text { Peter J. Hotez } \\ \text { Baylor College of Medicine }\end{array} & \text { David Heymann } & \text { Warren D. Johnson } \\ & \text { Health Protection Agency_London } & \text { Cornell University } \\ \mathbf{2 0 1 7} & \mathbf{2 0 0 7} & \mathbf{1 9 9 3} \\ \text { Scott O'Neill } & \text { David Sack } & \text { Robert E. Shope } \\ \text { Monash University } & \text { Johns Hopkins University } & \text { Yale Arbovirus Research Unit } \\ \mathbf{2 0 1 5} & \mathbf{2 0 0 5} & \mathbf{1 9 9 1} \\ \text { Eleanor Riley } & \text { Didier Raoult } & \text { Donald Hopkins } \\ \text { London School of Hygiene and Tropical } & \text { Unite des Rickettsies Marseilles, France } & \text { Global 2000 } \\ \text { Medicine } & & \\ \mathbf{2 0 1 3} & \mathbf{2 0 0 3} & \mathbf{1 9 8 7} \\ \text { Frank Richards } & \text { Philip Russell } & \text { Leonidas Deane } \\ \text { The Carter Center } & \text { DHHS Office of the Assistant Secretary } & \text { Instituto Oswaldo Cruz } \\ \mathbf{2 0 1 1} & \text { for Public Health Emergency } & \\ \text { Anita Zaidi } & \text { Preparedness } & \\ \text { Aga Khan University } & \mathbf{2 0 0 0} & \end{array}$

\section{Recognitions}

\section{AWARD FOR LEADERSHIP IN ADVOCACY FOR VACCINES}

Peter J. Hotez

National School of Tropical Medicine

Baylor College of Medicine

\section{AWARD FOR EXTRAORDINARY LEADERSHIP IN THE FIGHT AGAINST MALARIA \\ 2006 \\ Victoria McGovern \\ The Burroughs Wellcome Fund \\ In recognition of contributions in the education and training \\ of researchers in the fields of parasitology and international \\ 2005 \\ Anthony Fauci \\ National Institutes of Allergy and Infectious Diseases, NIH \\ In recognition of commitment to the health and well-being of individuals throughout the world.}

medicine.

\section{CERTIFICATE OF RECOGNITION-}

To recognize corporations, foundations or other institutions or individuals who have made meritorious contributions toward the control of tropical diseases.

2002

The Pfizer Foundation

1999

Jean Hickman

National Institutes of Health

\section{RECOGNITION AWARD IN GLOBALHEALTH}

\section{3}

Rear Adm. Tim Ziemer, United States Navy(Retired)

U.S Global Malaria Coordinator

\section{4}

The Rockefeller Foundation

\section{7}

Harold Varmus

National Institutes of Health

\section{DISTINGUISHED SERVICE AWARD-}

In recognition of the Foundation's global efforts to prevent and control tropical infectious diseases.

Bill \& Melinda Gates Foundation. . . . . . 2000 


\section{AMERICAN COMMITTEE ON ARTHROPOD-BORNE VIRUSES (ACAV)}

\section{ACAV 2019 Annual Council Meeting Minutes \\ Prepared by Shannon Bennett \\ Gaylord Hotel and Convention Center \\ National Harbor, MD \\ November 20, 2019 \\ Chair: Lark Coffey \\ Secretary: Shannon Bennett \\ Treasurer: Rebecca Christofferson \\ Chair-Elect: David Morens}

\section{General:}

- ACAV chair noted that $\$ 10,000$ revenue to ACAV is confirmed from parent society ASTMH by June 30 but with no roll-over to next year. One suggestion for the left-over funds was to confer additional travel awards, however funds need to be spent too far in advance of the meeting for this to be a viable solution. Instead, this past year's remaining funds were invested in designing a new ACAV logo and printing it on promotional products. The new logo was voted on over email by council members leading up to the annual meeting.

- ACAV hosted an informational table at the ACAV symposium as well as in the Exhibit Hall that featured new logo-embossed promotional products (T-shirts, mugs, pens, and pins).

- ACAV membership is down by 7\%: Membership in 2019 was 416 , down from 446 prior year.

- Trainees are converting to full membership at a rate of $8 \%$.

- Council identified the need for membership growth as well as revenue, particularly to bring in more young people. Ideas included identifying student-friendly activities/support, and/or combine ACAV meetings with other society meetings such as ASV or ACME.

\section{Strategic Plan 2020 Goals and Objectives:}

- Position ACAV to serve as the leading professional subgroup advancing knowledge of arboviruses globally

- Expand educational offerings from ACAV members

- Augment ACAV member value by serving as the primary site for professional interactions between arbovirologists

- Expand the global influence of ACAV

- Increase training opportunities in arbovirology

- Strengthen the financial position of ACAV
- To advance the strategic goals, the council discussed:

- Producing/distributing a newsletter

- Setting-up and managing an ACAV email and list-serve

- ACAV website upgrade:

Current Website:

Awards

Executive Council Members

Newsletter

History

Governance

Proposed Website:

Current+

Mission \& Vision Statement

Goals \& Objectives

Membership Directory

Become a member

Purchase ACAV memorabilia

Donate

Where does my ACAV donation go?

Educational Offerings

Newsletter

Photos

Videos by ACAV members

Affiliate groups

Books by ACAV members

ACAV in the news

Recent and hot ACAV publications

ACAV trainee spotlight \& publications

Job postings

Clinical and Educational Resources

ARBOCAT

Historical archives

News and events

Social Media

- Representation on ASTMH's Expert Working Group:

Expert Working Group Proposal: The ASTMH should create a sitting expert "pandemic/epidemic response group" comprised of 10-15 senior experienced thought leaders involved in international science and health, and representing diverse disciplines including medicine, public health/epidemiology, virology and bacteriology, veterinary medicine, and one-health and ecological approaches, among others. The experts would also, as a group, have working collaborative relationships with key players in the countries where epidemics occur including locales in Africa, Southeast Asia/Asia, and South \& Central America and the Caribbean. Some members should be scientists 


\section{AMERICAN COMMITTEE ON ARTHROPOD-BORNE VIRUSES (ACAV)}

working abroad in countries where such diseases emerge. The experts should generally have worked with the major pandemic (e.g. influenza) and high consequence diseases (e.g., hemorrhagic fevers, diseases caused by coronaviruses like SARS and MERS). Experts in selected bacterial (e.g., cholera) and parasitic (e.g., malaria) diseases should be included. Membership should rotate approximately every two years.

The group would meet every 1-2 months via phone and immediately/on an as-needed basis when an important disease appears. Top ASTMH experts in particular emerging diseases would be quickly added to the group for the purpose of dealing with that emerging disease, including representatives of US federal agencies such as CDC and $\mathrm{NIH}$.

The group would do the following: 1) identify emerging epidemics and draw attention to them; 2) develop consensus opinions on epidemic response needs, resources available, and possible approaches; 3 ) identify in-country and international players and work with them; communicate expert opinion to the press and public via press releases, media availabilities, and if needed position papers or other expert opinions; 4) identify ASTMH members, both in-country and otherwise who may be available to work in epidemic response partnerships; 5) if funding becomes available, create a dedicated fund to be used to support epidemic responses especially in-country support of science and ministry resources and coordination, and linking public health and science expertise. For example, one or more experts might be dispatched to work with the Ministry in local response, coordination, or policy decisions. (From Joel Breman in discussion with David Morens).

- Collecting stories/articles for AJTMH newly launched "Stories from the Field:"

Announcing Stories From the Field in the American Journal of Tropical Medicine and Hygiene: The Journal is introducing a new category of papers titles, "Stories From the Field." This category will highlight descriptive personal stories based on author's experiences practicing clinical tropical medicine, performing tropical medicine research, taking part in international health education, and/or delivering global health services. Preference will be given to articles that provoke thought, encourage new ideas, share innovative approaches, promote sustainability, highlight local efforts, or work to braoden perspectives of Journal readers.

All articles should inform our readers about the world, people, health, hygiene, disease, and the myriad of issues that impact tropical medicine and global health. Through sharing these stories, it is hoped that we can advance our thinking and improve the quality of research and healthcare that we proved. Submission will be accepted through the Journal site at https://mc.manuscriptcentral.com/ajtmh.

Essays should be title but have no section headings. The maximum length is 1500 words. The maximum number of authors is 3. If patients are discussed, they should be deidentified. Preapproval is not required before submission, but potential authors are welcome to request feedback on suggest topics before submission.

- Distributing an open-access ACAV Membership Directory

- Curating \& preserving historical documents, with the following subgoals:

- Archiving materials

- Cataloguing entries

- Digitization of content

- Address permanent safe appropriate storage

- Project could cost $\sim \$ 300,000$, for which we would need a grant (IMLS?)

- Current status:

- Tom Monath has put out a call for archival donations

- Scott Weaver saw to the collecting and depositing of physical boxes of ACAV documents

- Another priority is to better define roles for the councillors. The following tasks could be assigned to councillors and/or are priorities:

\section{Treasurer's Report:}

Revenue from dues (\$1900) and ASTMH allotment $(\$ 10,000)$ offset expenses from travel awards (7 at $\$ 500$ each) and the Batnayas travel award (\$2500) plus meeting expenses TBD.

\section{Report from Subgroups:}

Subcommittee on Information Exchange (SIE)

- Working on catalogue and updating it

- Newsletter also in production

- Social media seen as an important mechanism for information exchange and in need of the right person to take it on

Subcommittee on Interrelationships Among Catalogued Viruses (SIRACA)

- ICTV undergoing big changes, ACAV needs representatives on subgroups

- EEE outbreak brought to light public health shortcomings

- Evaluation of arbovirus status 


\section{AMERICAN COMMITTEE ON ARTHROPOD-BORNE VIRUSES (ACAV)}

Subcommittee on Arbovirus Laboratory Safety (SALS)

- A substantial discussion was had around producing a safety paper describing laboratory-acquired infections (LAIs) including whether said paper would be doable and strategic.

SEAS

- No report at this time

Next steps include deciding the succession of ACAV leadership:

- Chair-Elect: we did not select a chair-elect for 2021 at the time of the meeting and have instead put out a call for nominations by email; vote will be handled by email.

\section{Business Meeting Highlights:}

Thank you to outgoing Trainee Councilor Maria Onyongo Welcome incoming Councilors:

Patricia Aguilar

Nathan Grubaugh

Trainee Councilor, Amy Krystosik

\section{ACAV Activities at ASTMH:}

Arbovirology (ACAV) Pre-Meeting Course: ArboviromicsHow 'omics' Technologies are Advancing Arbovirus Research and Control

Organized by Louis Lambrechts and Felix Hol

November 20

Panel Discussion: Career Pathways in Science and Identifying Your Niche

Organized by Maria Onyango, trainee councilor

November 21

ACAV Symposium I: ACAV Business Meeting, Award Presentations and Research Presentations by Award Recipients

November 22

ACAV Symposium II: Everything Old Is New Again: The ReEmergence of Yellow Fever

Organized by Ann Powers and Desiree LaBeaud

November 22

TropStop Career Chats: How to ace that grant application Organized by Maria Onyango

November 22

Speed Networking with the Experts

November 22

\section{AMERICAN COMMITTEE ON ARTHROPOD- BORNE VIRUSES (ACAV) ACAV Executive Council Members-2020}

Member/Position Term Expires

David Morens, Chair .................................... 2020

Lark Coffey, Past-Chair .................................. 2020

Patricia Aguilar, Chair-Elect......................... 2020

Shannon Bennett, Secretary ....................... 2020

Rebecca Christofferson, Treasurer............... 2020

Laura Kramer, Councilor ................................. 2020

Desiree LaBeaud, Councilor .......................... 2020

Rebecca Christofferson, Councilor ................ 2021

Lark Coffey, Councilor .................................... 2021

Thomas Ksiazek, Councilor............................ 2021

David Morens, Councilor................................ 2021

Shannon Bennett, Councilor .......................... 2022

Louis Lambrechts, Councilor ........................ 2022

Patricia Aguilar, Councilor ............................ 2023

Nathan Grubaugh, Councilor ....................... 2023

Amy Krystosik, Councilor for Trainees........... 2020

Ann Powers, Ex Officio Chair SIE ................... 2019

Thomas Ksiazek, Ex Officio Chair SALS.......... 2019

Mike Turell, Ex Officio Chair, SEAS ............... 2019

Nikos Vasilakis, Ex Officio Chair, SIRACA ....... 2020

\section{GUIDELINES FOR THE AMERICAN COMMITTEE OF ARTHROPOD-BORNE VIRUSES}

During the 1987 executive council meeting of the American Committee on Arthropod-borne Viruses (ACAV) in Los Angeles, it was agreed that guidelines for the committee need to be established and recorded in a single document. The ACAV currently operates under procedures that are stated in various reports. A summary statement of the ACAV organization would assist new executive council members and chairpersons in understanding how the committee operates. In addition, modifications in procedures could easily be incorporated into a centralized body of information.

The information that follows is intended only as a guide for the ACAV organization; goals of the ACAV are stated in other publications. During the formation of ACAV it was agreed that the group would not be a formal organization with by-laws. Rather, it would provide a forum for exchange of information among people interested in arbovirus research.

The ACAV meets annually in association with the American Society of Tropical Medicine and Hygiene (ASTMH). An executive council is the governing body of the ACAV. The council is chaired by a person who directs council and committee meetings and retains the ACAV files. The chairperson of the executive committee serves a 4-year renewable term and is elected by the executive council at the 


\section{AMERICAN COMMITTEE ON ARTHROPOD-BORNE VIRUSES (ACAV)}

annual council meeting. The council consists of six people who each serve a 4-year term. New council members are elected by members of the ACAV. Council members who are rotating off form a search committee that nominates council candidates; the slate of candidates must be approved by the existing council. The chairperson of the council sends ballots to the ACAV membership. The candidates who receive the most votes are notified and take office at the next annual meeting of the ACAV. There is no quorum for the election of officers or for other council and committee votes. Should a member of the executive council resign or leave the ACAV, a new council member is appointed by the executive council to complete the original member's term.

Other ACAV positions include a secretary, treasurer, historian and international advisors. The secretary is the newest member of the council and records minutes of the council meeting. The treasurer is appointed to a 4-year renewable term by the executive council and maintains the ACAV funds in a subaccount of the ASTMH. Withdrawals and deposits from the ACAV account are arranged with the treasurer. The council chairperson must approve all withdrawals,

The historian is designated by the council and provides historical information on the operation of the ACAV. The council selects international advisors, determines the number of advisors, and defines the length of the advisor's terms.

All people interested in joining the ACAV may do so by attending a meeting and signing their name to the attendance sheet. Membership in the ASTMH is not a requirement for membership in the ACAV. People missing three consecutive meetings will lose their membership in the ACAV. Exceptions to this attendance rule are made for people with emeritus status and for some overseas participants. Emeritus status is automatic upon retirement. The executive council must approve an attendance exception for people living overseas.

Subcommittees are an important part of the ACAV and are formed when sufficient interest is presented to the executive council by ACAV members. The council reviews proposed subcommittees and decides whether a particular subcommittee is needed. When council approves the formation of a subcommittee, it appoints a chairperson. The subcommittee chairperson, in consultation with the ACAV chairperson, appoints subcommittee members. New subcommittees formalize their objectives and structure. All subcommittees report progress on objectives at the annual meeting to the ACAV.

The ACAV presents three awards, when appropriate, in staggered three-year intervals. The chair of the executive council appoints a nominating committee for each award. Recipients of awards are selected by the executive council. An announcement requesting nominations for each pending award is published in the Tropical Medicine and Hygiene News at least six months prior to the ASTMH annual meeting. This procedure provides the nominating committee with nominations and documentation from ACAV members. The Scherer/Hardy Award is given to an outstanding graduate either before graduation or up to 3 years post-graduation. Accrued interest

\section{Awards}

\section{Richard M. Taylor Award}

Presented to a person who has made outstanding contributions to arbovirology throughout his or her career.

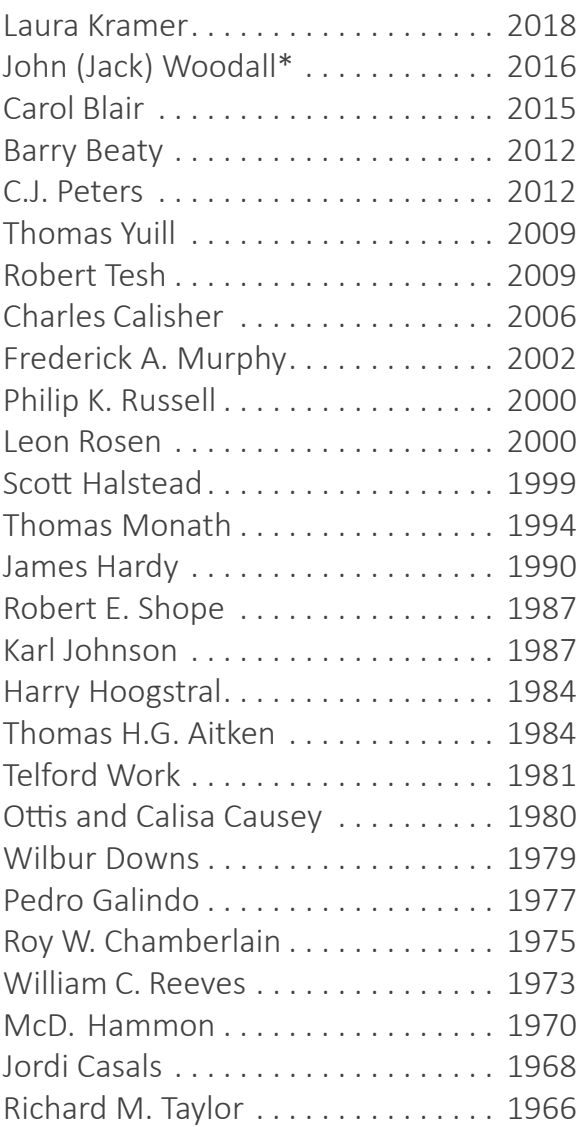

\section{Dalrymple/Young}

Gregory Ebel . . . . . . . . . . . . . . . 2019

Kathryn Hanley . . . . . . . . . . . . . 2016

Ebihara Hidecki . . . . . . . . . . . . 2013

Jonathan Towner . . . . . . . . . . . . 2010

Heinz Feldmann ............. 2005

Scott C. Weaver. . . . . . . . . . . . 2002

Stuart T. Nichol . . . . . . . . . . . . . 1999

Connie Schmaljohn. . . . . . . . . . . . 1996

James LeDuc . . . . . . . . . . . . . . . . 1990

*Given out of rotation 
Donald Burke. . . . . . . . . . . . . . . 1990

Barry Beaty . . . . . . . . . . . . . . . 1988

Thomas Monath . . . . . . . . . . . . . 1985

David Bishop . . . . . . . . . . . . . . . 1982

\section{Scherer/Hardy Award}

Nathan Grubaugh . . . . . . . . . . . . 2018

Farooq Nasar . . . . . . . . . . . . . 2015

Alexander Ciota. . . . . . . . . . . . . . 2013

Amy Schuh. . . . . . . . . . . . . . . . . 2013

Brian Bird. . . . . . . . . . . . . . . . 2010

Patricia V. Aguilar . . . . . . . . . . . . 2007

Gregory D. Ebel. . . . . . . . . . . . . . 2004

Aaron Brault . . . . . . . . . . . . . . . 2001

Ann M. Powers . . . . . . . . . . . . . . . 1997

Kurt Kamrud . . . . . . . . . . . . . . . . . 1997

Scott C. Weaver. . . . . . . . . . . . . . . 1993

George Ludwig . . . . . . . . . . . . . . 1989

Rebeca Rico-Hesse . . . . . . . . . . . . 1986

Daniel Sundin . . . . . . . . . . . . . . 1983

\section{ACAV Student Travel Award}

Priscila Castanha. . . . . . . . . . . . . 2019

Marissa Childs. . . . . . . . . . . . . . . 2019

Francesca Falconi . . . . . . . . . . . . 2019

Siew Wai Fong. . . . . . . . . . . . . . . 2019

Cat Lippi. . . . . . . . . . . . . . . . . . . 2019

Alice Michie. . . . . . . . . . . . . . . . . 2019

Blake Schouest . . . . . . . . . . . . . . 2019

Juan Aguilar. . . . . . . . . . . . . . . . . . 2018

Nikita Cudjoe. . . . . . . . . . . . . . . . . . 2018

Laura Dickson . . . . . . . . . . . . . . 2018

Danilo Lemos. . . . . . . . . . . . . . . . 2018

Daniela Michlmayr . . . . . . . . . . . 2018
Ranya Mulchandani . . . . . . . . . . 2018

Nivison Nery, Jr . . . . . . . . . . . . . . 2018

Maria Onyango . . . . . . . . . . . . . . . 2018

Nunya Chotiwan . . . . . . . . . . . . . . 2017

Amy Krystosik . . . . . . . . . . . . . . . 2017

Carla Mavian . . . . . . . . . . . . . . . . . 2017

Erin McDonald . . . . . . . . . . . . . . 2017

Wen Yang Tsai . . . . . . . . . . . . . . . . 2017

Joseph Fauver . . . . . . . . . . . . . . . 2016

Claire Heath. . . . . . . . . . . . . . . . . 2016

Devika Sirohi . . . . . . . . . . . . . . . 2016

James Weger. . . . . . . . . . . . . . . 2016

Albert Auguste . . . . . . . . . . . . 2015

Rebecca Hamlin . . . . . . . . . . . . . 2015

Lewis Hun . . . . . . . . . . . . . . . . . 2015

Stacy Scroggs. . . . . . . . . . . . . . . . 2015

Nunya Chotiwan . . . . . . . . . . . . . . . 2014

Brittany Dodson ... . . . . . . . . . . . 2014

Nisha Duggal . . . . . . . . . . . . . . . . . 2014

Nathan Grubaugh. . . . . . . . . . . . . 2014

Meghan Elizabeth Hermance . . . . 2014

\section{Kelly Labell Travel Award}

Nicholas Bergren. . . . . . . . . . . . . 2013

Andrea Bingham . . . . . . . . . . . . . 2012

Patrick Vander Kelen. . . . . . . . . . . . 2011

Sheri Anderson . . . . . . . . . . . . . 2010

Nicole Arrigo . . . . . . . . . . . . . . . . 2009 


\section{ACME SUBGROUP (AMERICAN COMMITTEE OF MEDICAL ENTOMOLOGY)}

\section{Council Meeting Minutes \\ Wednesday November $20^{\text {th }}, 2019$, 4:00 pm - 5:30 pm}

Chesapeake 1; Gaylord National Resort and Convention Center

National Harbor, Maryland USA

Chair: Matt Thomas, PhD

Secretary/Treasurer: Alvaro Molina-Cruz, PhD

Past Chair: Phil Armstrong, PhD

\section{Call to Order (Matt Thomas)}

\section{4:00-4:05 Treasury Report.}

Alvaro- The total net assets for all ACME funds were $\$ 24,801$ for the period ending in June 30, 2019. The revenue from memberships and member donations (including donations at the 2018 ACME booth) for the period July 2018- June 2019 was $\$ 5,325$. The ASTMH Board donated a $\$ 10,000$ grant to ACME toward its initiatives during 20182019, as it did in the previous year. The Board donation was used to cover three 2018 ACME Student Travel Awards (\$2,700 total), 2018 ACME Future Leaders Fellowship in International Medical Entomology $(\$ 2,000)$, the publication of the Arthropod Containment Guidelines $(\$ 3,200)$, scholarship funds for the 2019 Biology of Vector-borne Diseases course at U. of Idaho $(\$ 1,712.93)$ and the 2018 Speed Networking Event (\$387.07). ACME received a generous contribution $(\$ 10,000)$ from S.C. Johnson \& Son Inc. toward the Breakthrough in Medical Entomology Award and Future Leaders Fellowship. This finishes the commitment by S.C. Johnson, ACME should work on identifying other donors. ACME continued to avoid expenses associated with the council meeting from \$406.80 in 2017 to $\$ 0.00$ in 2018.

\section{4:05-4:20 Council Elections.}

Introduction of New Council Members, Eleanor Sternberg, Jennifer Stevenson and Marco Neira.

Introduction of Trainee Member, Olivia Winokur.

Nomination of Chair-Elect. Gabriel Hamer was the only nominated person for chair; voted on Gabrielelected.

Nomination of Secretary/Treasurer. Molly Duman Scheel was the only nominated person for Secretary/Treasurer; voted on Molly- elected.

Suggestions for council members, 2020 will be presented by outgoing councilors (Alvaro Molina-Cruz, Diana Ortiz and Laura
Harrington) that will contact the candidates to confirm their interest. The list of candidates should be finalized by March 2020.

Task Force Updates.

4:20-4:30 AWARDS update was presented by Matt Thomas.

Members. UPDATE Phil Armstrong, Kate Altman, Laura Harrington, Matt Thomas. MEMBERSHIP FOR 2020 was identified: Laura Harrington, Matt Thomas, Ellen Dotson, Gabe Hamer.

It was agreed that Ellen (chair) will oversee the Hoogstraal award selection, Matt (past-chair) the student travel awards and Gabe (incoming chair) the Breakthrough in Medical Entomology award.

\section{Accomplishments.}

- The 2019 \$10K from the ASTMH Board allowed us to give 2 grad-student awards (\$900 each), 2 postdoc awards (\$900 each) and 2 international (\$2000 each). The total amount is $\$ 7,600$. The remaining funds were used to fund scholarships for the Vector Biology Course in U. Idaho.

- The honorees for 2019 were: Hoogstraal Award, Frank Collins, U. Notre Dame; Breakthrough in Medical Entomology Ward, Stephanie James, Foundation for the National Institutes of Health; Future Leaders Fellowship in International Entomology, Adélaïde Miarinjara, National Institutes of Health; Travel Award for Young Investigators, Diana Nyanting'a, Kenya Medical Research Institute; Kristine Werling, Harvard School of Public Health; Young Investigator Award-International, Renee Ali, University of West Indies; Marilyn Murindahabi, University of Rwanda; Young Investigator Award-Post Doc, Deepani Fernando, University of Peradeniya, Sri Lanka; Emily Gallichotte, Colorado State University.

\section{Challenges.}

- Number of applicants for the International Leaders were 5 while there were only 2 applicants for the Breakthrough Awards for 2019. Several options to help increase the number of applicants were discussed: facilitate the nomination by allowing self-nomination with an external 
letter of support, increase the visibility of the awards in other events and social media, allow nomination and letters by non-members, the ACME council could suggest nominations, and allow nomination of multiple individuals involved in a single breakthrough finding. These changes will be incorporated in the ACME website with the rules for award application

- SC Johnson gave their last donation to ACME (\$10K) on Dec 2018, there is a need to identify other possible donors for awards.

\section{4:30-4:40 TRAINEES.}

Members. Diana Ortiz, Andrew Golnar, Matt

Thomas.

MEMBERSHIP FOR 2020 - Diana Ortiz, Olivia Winokur, Ellen Dotson.

\section{Accomplishments.}

- $\quad$ There were 150 students and 11 mentors registered for the Speed Networking and ACME/ACAV Lunch Networking events.

\section{Challenges.}

- Mentor identification has been a challenge. Currently the list of potential mentors is provided by Koya Allen (ASTMH Trainee Committee).

\section{Goals. Suggestions.}

- There was a discussion on ways to to engage and support trainees in addition to the networking events and travel awards. The task force will contact trainees to get their input on other activities they would like to have through ACME. The task force is also planning some trainee-trainee pairing outside the speed networking event. Another possibility is to organize and sponsor a social event outside the meeting for networking together with ACAV trainees. The task force is planning a mentor-mentee program beyond the speed networking event.

\section{4:40-4:50 ADVOCACY.}

Members. Phil Armstrong, Ellen Dotson

MEMBERSHIP FOR 2020- Ellen Dotson

Accomplishments.

- Arthropod Containment Guidelines publication.

\section{Challenges.}

Goals. Suggestions. There was discussion to to join intitiatives with other organizations like AMCA, ESA, PAMCA, Vector Borne Disease Coordinated Network. Ellen will contact some of these networks. ACME could do some international activities with PAMCA.

4:50-5:00 VISIBILITY.

Members. Alvaro Molina-Cruz, Gabe

Hammer

- MEMBERSHIP FOR 2020- Gabe Hammer, Molly Duman Scheel.

Accomplishments.

- $\quad$ Two newsletters were published in 2019. Gabe and Molly will work on the 2020 newsletters.

- $\quad$ Booth, 2018 booth sales/donations.

Challenges.

- $\quad$ Enhancing visibility in the digital age. ACME has not used social media. It was suggested that Twitter might be the best one to try.

- There is still lack of use of MyCommunities by members.

Goals. Suggestions.

- Rebecca Hamel, is the new Development Manager (ASTMH). Rebecca has a strong background in global health resource development. Rebecca can help ACME in strategies to increase non-dues revenues.

5:00-5:10 PRE-MEETING COURSE.

Members. Phil Armstrong, Laura Harrington, Chris Barker

- MEMBERSHIP FOR 2020 Laura Harrington, C Accomplishments. The previous premeeting course of Medical Entomology for clinicians was successful.

Challenges.

Goals. Suggestions. The premeeting course proposed for 2020 will be again on Medical Entomology for clinicians. Laura Harrington volunteered to organize it.

5:10-5:20 Updates from Executive Council.

- The parent Board has suggested preparation of strategic plans by subgroups. ACME will work on that based on the Board guidelines and ACAV strategic plan.

5:20-5:30 Open Discussion.

- Overall goals and suggestions

\section{Calendar Items for ACME-associated events:}

\section{WEDNESDAY Nov 20}

4:00 pm- 5:30 pm, ACME Council Meeting, Chesapeake 1 (Ballroom Level). 
THURSDAY Nov 21

8:00 am - 9:45 am, American Committee of Medical Entomology (ACME) Symposium I: Annual Business Meeting, Awards and Hoogstraal Medal Presentations and Networking Reception, in Potomac D (Ballroom Level).

10:15 am - 12:00 pm, American Committee of Medical Entomology (ACME) Symposium II: Will History Repeat Itself? Lessons Learned from Previous Vector Control

Efforts, in Potomac D (Ballroom Level)

12:00 pm- 1:30 pm, ACME Networking Lunch, in National Harbor 8.

\section{Friday Nov 22}

5:00 pm-6:45 pm, $7^{\text {th }}$ Annual "Speed-Networking with the Experts", in Riverview Ballroom 1/2/3. 


\section{AMERICAN COMMITTEE OF MEDICAL ENTOMOLOGY (ACME)}

\section{AMERICAN COMMITTEE OF MEDICAL ENTOMOLOGY (ACME) CHARTER}

Name. The name of the organization shall be the American Committee of Medical Entomology (acronym-ACME). It is organized under the auspices of the American Society of Tropical Medicine and Hygiene (ASTMH), and will meet at least annually in conjunction with that society's annual meeting.

Objectives. The objectives of ACME shall be as follows

- Topromote medical entomology in the ASTMH and in organizations whose scopes of activities include the area of human diseases transmitted by arthropods.

- To organize symposia or workshops annually that emphasize, but are not limited to, the contributions of medical entomology to tropical medicine.

- To encourage active participation of medical entomologists in the ASTMH.

- To recognize outstanding contributions by medical entomologists.

Membership. Membership is effective for one year and shall result from either of two acts: 1 . Payment of a fee of $\$ 25$ to the ASTMH designated for ACME membership. (Funds shall be placed in the ACME account [1].); 2. Students and post-doctoral fellows may become ACME members by signing up and are not required to pay ACME membership dues. All ACME members must be in good standing as members of the ASTMH.

Officers. There shall be an Executive Council comprised of twelve [12] members, including a Chairperson, a Chairperson-elect, a Past- Chair, and a Secretary-Treasurer. Officers must be ACME members at the time of their election. Executive Council members serve four-year terms, and three new members shall be elected each year. Terms of service begin with the Executive Council meeting held the year of election and end with the Executive Council meeting at the end of the fourth year i.e. Executive Council members attend five meetings. The Chairperson, Past-Chair and Chairperson-elect serve for one year. The Secretary-Treasurer shall serve for a period of one to three years. The Past-Chair is an ex officio member of the Executive Council without Council voting rights if they have exceeded their fourth year of Council membership.

\section{SELECTION OF OFFICERS:}

Executive Council members. The three members who are due to rotate off the Executive Council shall comprise the nominat- ing committee each year. They shall meet at the beginning of their third year of service immediately following the annual ACME business meeting and prepare a slate of nominees for election to the Executive Council. This slate shall consist of the names of at least five individuals. Nominees must be ACME members at the time of their nomination and must either have not previously served on the Executive Council OR one of the following is true:

- They are presently filling unexpired vacancies created by death or resignation from the Executive council

- They have been off the Executive Council for at least one full year

It shall be the responsibility of the Nominating Committee to deter- mine the eligibility of the nominees and to ascertain their willingness to serve on the Executive Council if elected. Upon completion of the slate of nominees and ascertaining the willingness of the nomi- nees to serve, the Nominating Committee shall submit the slate to the Secretary-Treasurer, who shall prepare ballots to be sent to all members. Election should be completed no later than July 1 following the annual business meeting. Executive Council members elected shall assume office at the beginning of the following Executive Council meeting.

Chairperson-Elect. The Executive Council shall elect a Chairperson- Elect from among their members during the annual meeting of the Executive Council. Service as Chairperson-Elect begins at the end of the next ACME Business meeting.

Chairperson. The serving Chairperson-Elect shall assume the res- ponsibilities of the Chairperson when the newly elected Chairperson- Elect assumes office.

Past-Chair. The Chairperson from the previous year shall serve as the Past-Chair.

Secretary-Treasurer. The Executive Council shall elect a Secretary Treasurer (which may include self-nominations) from among their members at any time there is a pending vacancy of the office. Election shall be for a period of no more than three years and is contingent upon annual election by the Executive Council.

\section{DUTIES OF OFFICERS.}

Chairperson. The Chairperson shall represent ACME to other Organizations, shall officiate at all meetings of the Executive Council and at annual business meetings, shall submit an annual report to the Executive Council of ASTMH, make all appointments for ACME, such as the individual to present the Hoogstraal Medal for Outstanding Achievement in Medical Entomology. 


\section{AMERICAN COMMITTEE OF MEDICAL ENTOMOLOGY (ACME)}

Chairperson-Elect. The Chairperson-Elect shall assist the Chair-person, shall serve in the absence of the Chairperson, and shall be responsible for the organization of symposia and workshops at the annual meeting of the ASTMH.

Past-Chair. The Past-Chair shall serve as a Councilor to the Chairperson, particularly to maintain ACME institutional memory in council actions and decisions. They may also be called upon by the Chairperson to attend the ASMTH Executive Council Annual and Mid-year meetings in the Chairperson's stead if needed.

Secretary-Treasurer. The Secretary-Treasurer shall maintain the membership rolls, shall record minutes of all meetings, shall prepare and mail ballots for election, and shall receive, disburse and maintain an accounting of all moneys used by ACME. The Secretary-Treasurer keeps records of the council members and officers and shall notify Executive Council members of the years of the meetings they are expected to attend.

\section{OTHER PROCEDURES.}

Subcommittees. Subject area subcommittees can be established and abolished by the Executive council.

Unusual vacancies. In the event of vacancies created on the Executive Council by death or resignation, additional nominations will be made by the members of the Executive Council and will be included on the annual ballot for vote by the membership to complete the unexpired terms. In such cases, individuals receiving the next highest number of votes to the three individuals elected to full terms will be elected to complete the unexpired terms.

Adoption of subsequent modifications. The adoption of this docu- ment and subsequent changes shall be upon recommendation of the Executive Council and affirmation by a simple majority of those members present at the annual ACME business meeting and will be effective immediately.

\footnotetext{
1 Accepted by those attending the ACME business meeting at the 2003 ASTMH National meeting in Philadelphia.

2 In 1990, the membership voted (90 for; 11 against) to increase the term of membership on the Executive Council of the American Committee of Medical Entomology from three to four years. As a result, the number of persons serving on the Executive Committee at any given time will be 12 .

3 This revised charter was accepted unanimously by a vote of those attending the ACME business meeting at the 2012 ASTMH Annual Meeting in Atlanta.
}

ACME Executive Council Members 2020

Member/Position Term Expires

Ellen Dotson, Chair ...................................................2020

Gabriel Hamer, Chair-Elect ...........................................2020

Matthew Thomas, Past-Chair .................................... 2020

Molly Duman Scheel, Secretary/Treasurer....................2020

Laura Harrington, Councilor ............................................. 2020

Matthew Thomas, Councilor ........................................ 2020

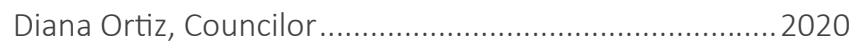

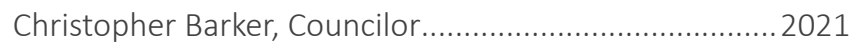

Ellen Dotson, Councilor .............................................. 2021

Gabriel Hamer, Councilor ............................................... 2021

Molly Duman Scheel, Councilor ...................................... 2022

Audrey Lenhart, Councilor............................................ 2022

Douglas Norris, Councilor ......................................... 2022

Marco Neira, Councilor........................................... 2023

Eleanore Sternberg, Councilor .......................................2023

Jennifer Stevenson, Councilor ..................................... 2023

Olivia Winokur, Student Representative........................ 2020

Matthew Thomas, Hoogstraal Medal Coordinator ..........2020

Laura Harrington, Awards Coordinator ......................... 2020
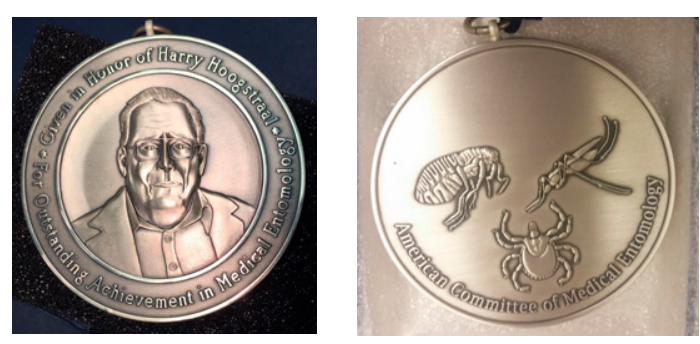

\section{Awards}

\section{Harry Hoogstraal Medal}

Frank Collins ... . . . . . . . . . 2019

Tom Scott. . . . . . . . . . . . . . . . . 2018

Willem Takken. . . . . . . . . . . . 2017

Patricia Nuttall. . . . . . . . . . . . . . . 2016

Durland Fish . . . . . . . . . . . . . . 2015

Robert Lane. . . . . . . . . . . . . . . . 2014

Barry Beaty . . . . . . . . . . . . . . . 2013

William Reisen. . . . . . . . . . . . . . . 2012

Abdu Azad . . . . . . . . . . . . . . . . 2011

Willy Burgdorfer . . . . . . . . . . . . 2010

William Collins. . . . . . . . . . . . . . 2009

Daniel Sonenshine . . . . . . . . . . . . 2008

Bruce Eldridge. . . . . . . . . . . . . . 2007

Mario Coluzzi . . . . . . . . . . . . . . 2006

Robert Washino ... . . . . . . . . . . 2005

John D. Edman ... . . . . . . . . . . . 2004

Andrew Spielman . . . . . . . . . . . . 2003

Michael Service. . . . . . . . . . . . . 2002

Chris Curtis ... . . . . . . . . . . . . . 2000

Gene R. DeFoliart . . . . . . . . . . 1998 
George B. Craig, Jr. . . . . . . . . . . 1996

A Ralph Barr . . . . . . . . . . . 1995

Thomas H.G. Aitken . . . . . . . . . 1993

James H. Oliver . . . . . . . . . . . . . . 1992

William L. Jellison . . . . . . . . . . . . 1991

William R. Horsfall. . . . . . . . . . . . 1990

Robert Traub . . . . . . . . . . . . . . 1989

Lloyd E. Rozeboom . . . . . . . . . . . 1988

William C. Reeves . . . . . . . . . . . 1987

ACME Breakthrough in Medical Entomology Award ACME presents this award for outstanding recent contributions (within the past 5 years) to the study and/ or practice of Medical Entomology that ultimately will contribute to reducing the burden of human diseases transmitted by arthropods. This award is designed to encourage and acknowledge significant advances in the field by investigators at any career stage.

Recipients

2019

Stephanie James

Foundation for the National Institutes of Health

\section{8}

Alvaro Molina-Cruz

National Institutes of Health

2017

Zhijian Tu

Virginia Tech

2016

Serap Aksoy

Yale University School of Medicine

ACME Future Leaders in International Medical Entomology Award

The Future Leaders fellowship is a competitive award that will be offered to an outstanding junior medical entomology researcher (must be at the undergraduate to post-doctoral level) to showcase individuals that have matched interests to ACME's objectives of promoting medical entomology and reducing the burden of human diseases transmitted by arthropods globally. Applicants must be a non-US citizen from a LMIC.

\section{Recipients}

\section{9}

Adélaïde Miarinjara

National Institutes of Health

\section{8}

Mabel Taracena

Universidad del Valle de Guatemala
Puja Tiwary

Banaras Hindu University

2017

Nsa Dada

Centers for Disease Control and Prevention

Eric Ochomo

Kenya Medical Research Institute

ACME Travel Awards for Young Investigators

Recipients

2019

Young Investigator Award - Graduate

Diana Nyanting'a

Kenya Medical Research Institute

Kristine Werling

Harvard School of Public Health

Young Investigator Award - International

Renee Ali

University of West Indies

Marilyn Murindahabi

University of Rwanda

Young Investigator Award - Post Doc

Deepani Fernando

University of Peradeniya, Sri Lanka

Emily Gallichotte

Colorado State University

2018

Young Investigator Award - Graduate

Leticia Smith

Cornell University

Young Investigator Award - International Fabian Aubrey

Institut Pasteur

Young Investigator Award - Post Doc

Sarah Merkling

Institut Pasteur

2017

Young Investigator Award - Graduate

Carolyn Hodo

Texas A\&M University

Young Investigator Award - International

Om Prakash Singh

Banaras Hindu University 


\author{
Young Investigator Award - Post Doc \\ Hannah Romo \\ Colorado State University \\ 2016 \\ Young Investigator Award - Graduate \\ Rebecca Love \\ University of Notre Dame \\ Young Investigator Award - International \\ Allan Muhwezi \\ Makerere University \\ Young Investigator Award - Post Doc \\ Laura Dickson \\ 2015 \\ Young Investigator Award - Graduate \\ Marissa Grossman \\ Emory University \\ Bahjat Marayati \\ University of North Carolina \\ Young Investigator Award - International \\ Marlon Saavedra Romero \\ Asociacion Benefica Prisma, Peru \\ Young Investigator Award - Post Doc \\ Claudia Rueckert \\ Colorado State University
}

\section{ACME Travel Award}

Marta Moreno Leirana. . . . . . . . . . 2014

Christina Newman . . . . . . . . . . . . 2014

Margaret Patermina Gomez . . . . . 2014

Robert McCann. . . . . . . . . . . . . . 2013

Anthony Clemons . . . . . . . . . . . . 2013

Julia Brown . . . . . . . . . . . . . . . . 2011

Cara Henry-Halldin . . . . . . . . . . . 2010

Win Surachetpong . . . . . . . . . . . . 2009

Lauren Cator . . . . . . . . . . . . . . 2009

Meera Venkatesan . . . . . . . . . . . 2008

Nicole Gottdenker. . . . . . . . . . . . . 2008

Maria Julia Dantur Juri . . . . . . . . . . 2007

Lisa Purcell. . . . . . . . . . . . . . . . . . 2007

Luca Facchinelli . . . . . . . . . . . . . . 2006

Sonja Kjos . . . . . . . . . . . . . . . 2006

Sharon Minnick. . . . . . . . . . . . . . . 2005

Rebekah Kent . . . . . . . . . . . . . 2005

Vincent Payet . . . . . . . . . . . . . . . 2004

Rebecca Robich. . . . . . . . . . . . . . 2004

Patricia Nkem Okoye. . . . . . . . . . . 2003

Junghwa Lim . . . . . . . . . . . . . 2003 


\section{ACMCIP SUBGROUP (AMERICAN COMMITTEE OF MOLECULAR, CELLULAR AND IMMUNOPARASITOLOGY)}

\section{$17^{\text {th }}$ Annual ACMCIP Business Meeting}

Friday, November 22, 2019

9:20-9:45am EST

Gaylord National Conference Center Room 327-329

ASTMH $68^{\text {th }}$ Annual Meeting, National Harbor, MD

1. President Mike Ferdig called the meeting to order, offering a welcome to all attendees and provided a brief overview of the ACMCIP subgroup.

2. We then held a memorial for two colleagues and friends whom we have lost over the past year: Shahid Khan and Alissa Myrick.

a. Steve Hoffman spoke in memory of Shahid, a "gentleman scholar" with an infectious smile and with whom he had worked for a number of years.

b. Christine Peterson spoke in memory of Alissa, with whom she shared their early trainee days and remained in touch during their careers and continued dedication to basic science and global health.

Both colleagues are survived by their children, families, and friends and colleagues around the world. May their memories be a blessing.

3. After a moment of silence in honor of our colleagues' memory, the President returned to business with ACMCIP 2019-2020 council elections. Two positions were open for nomination and confirmation vote from our Council meeting: President-elect and Trainee Councilor.

a. Mahalia Desruisseaux was nominated for the position of President-elect by Akhil Vaidya and the motion was seconded by Aabha Sharma. A vote was called by the President and passed unanimously.

b. Aabha Sharma was nominated for the position of Trainee Councilor by Amanda Lukens and seconded by Mahalia Desruisseaux. A vote was called by the President and passed unanimously.

c. A further announcement was made that we are seeking nominations for International Councilor as Christian Happi's term is ending.

i. Christy Petersen suggested Hugo Valdivia Rodríguez

ii. David Roos will follow-up with the President regarding another suggestion. He further suggested that we expand the number of
International Councilors on our executive board in order to better represent the large number of international scientists amongst our membership.

iii. Robin Stephens will follow-up with the President regarding another potential nominee.

iv. Keke Fairfax suggested Emmitt Jolly.

v. The President, Past-president, and President-elect will follow-up with each nominee.

4. The President then provided an update on 2019-2020 activities including a brief summary of our Council Meeting discussions (full Council Meeting minutes are included as an appendix):

a. Continued focus on ways to emphasize basic science research and parasitology as an integral component of our society.

b. Branding and identity, outreach and inclusion.

c. Empowering trainees, implementation of a new 'pipeline' leadership development committee

d. Explore the possibility of opening up an additional symposium slot (of our 6) to a second targeted symposium (e.g. trainee organized)

e. Draft plan for next year's ACMCIP symposium, led by Keke Fairfax: "Friend or Foe: The many faces of Myeloid cells in parasitic infections"

i. Additional suggestions from the audience included a symposium on the current limitations of culture systems for parasitic diseases: where we are, what challenges remain, and what do we need to cross the finish line?

f. 2020 Pre-Meeting Course

i. Represent translation of basic research to a field setting

ii. Possible foci to include pipelines for cutting edge vaccine/diagnostics

iii. Open discussion of additional ideas:

1. Culture systems for parasitic disease symposium idea could also be pursued as a pre-meeting course. This could then be coupled to assays/development of phenotypic assays.

2. The premeeting course should address challenges and opportunities for those working in disease endemic countries; e.g. immunodiagnostics

3. A general desire to include a practical/ hands-on component to the pre-meeting course was also emphasized

4. The cost of the pre-meeting course can be prohibitive for some - are we able 
to offer an award to help defray these costs, particularly for members from LMIC to be able to participate.

5. Celebration of 2019 Trager Award Recipient: Dr. David Horn, Professor of Parasite Molecular Biology, Biological Chemistry and Drug Discovery and the Wellcome Centre for Anti-Infectives Research (WCAIR), University of Dundee 


\section{AMERICAN COMMITTEE OF MOLECULAR, CELLULAR AND IMMUNOPARASITOLOGY (ACMCIP) 2020 Executive Council}

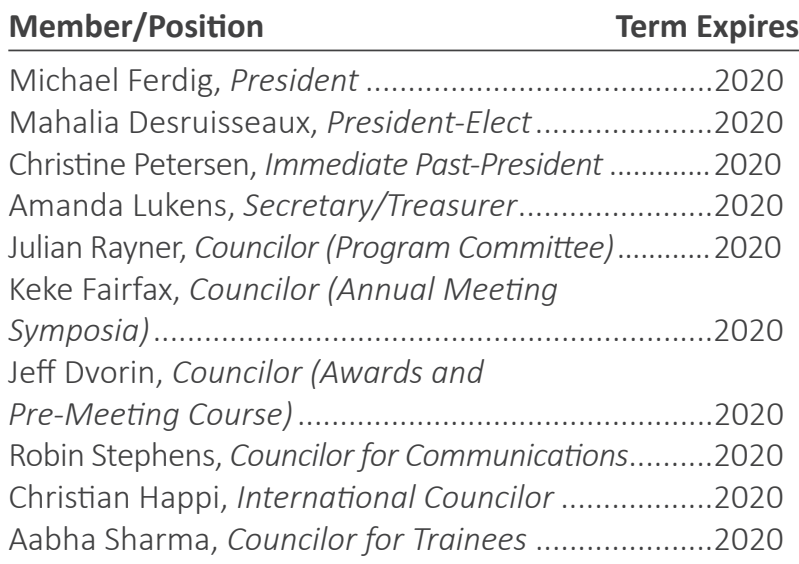

\section{CHARTER OF THE AMERICAN COMMITTEE OF MOLECULAR, CELLULAR AND IMMUNOPARASITOLOGY}

\section{Objectives}

The American Committee of Molecular, Cellular and Immunoparasitology (ACMCIP) is organized under the auspices of the American Society of Tropical Medicine and Hygiene (ASTMH) and has the following objectives:

- To promote an organization that facilitates interactions among scientists who work in the varied disciplines of parasitology, especially in basic laboratory, pre-clinical and translational research, clinician sciences and population-based sciences.

- Tofoster transfer of fundamental discoveries in basic research to applications that improve human health and to promote learning basic science aspects of parasitic diseases from humans afflicted with parasitic diseases.

- Tosponsor symposia or workshops to promote advanced research in medical parasitology.

- To facilitate recruitment and training in the most recent advances in medical parasitology.

- To recognize outstanding contributions to parasitology.

- To coordinate interactions and activities with other organizations and sponsored meetings to promote the objectives of ACMCIP.

- ACMCIP will meet at least annually under the aegis of the ASTMH Annual Meeting.

\section{Membership}

Membership will require attendance at the annual business meeting of ACMCIP, as certified by a sign-up sheet circulated at the meeting.

Established investigators will be assessed an annual fee of $\$ 25.00$ per year. No fee will be charged to post-doctoral or predoctoral trainees. Membership will continue unless a member is absent from three consecutive annual business meetings. A former member may reinstate membership by attendance at any subsequent annual business meeting of ACMCIP. ACMCIP will establish a fund for voluntary donations to be managed by ASTMH. Funds will be used for activities that further the objectives of ACMCIP, including receptions, annual awards, fellowships and international training opportunities, payment of page charges in the American Journal of Tropical Medicine and Hygiene for qualifying articles (eg. editorials, topical reviews, symposium proceedings, cutting-edge papers, etc.).

\section{Organization}

There will be an elected leadership in the form of an Executive Council. The membership of the Executive Council should reflect the wide diversity of ASTMH, include a diverse set of disciplines and be interna- tional in scope. There will be an elected Chair. Councilors will be elected to represent the major aspects of molecular, immunological, biochemical, cell biological and clinical field aspects of medical parasitology. There will be an annual business meeting of ACMCIP during the ASTMH Annual Meeting. Administration of ACMCIP will be done in close relationship to the ASTMH office and the chair of the program committee. Subcommittees will be formed to deal with important issues such as awards, training initiatives, fundraising, interfacing with the American Journal of Tropical Medicine and Hygiene, planning of ACMCIPsupported symposia and workshops at the ASTMH Annual Meeting, to coordinate the selection of speakers from the Woods Hole Molecular Parasitology meeting and the Woods Hole Immunoparasitology meeting to present at the ASTMH Annual Meeting. 


\section{AMERICAN COMMITTEE ON MOLECULAR, CELLULAR AND IMMUNOPARASITOLOGY (ACMCIP)}

\author{
Awards \\ ACMCIP Exchange Fellowship \\ Award Recipients \\ 2019 \\ Jessica Schue \\ Johns Hopkins University \\ 2018 \\ Martha Guevara Becerra \\ Universidad Peruana Cayetano Heredia \\ Anurag Kushwaha \\ Banaraas Hindu University
}

ACMCIP Award for Low and Low Middle Income Trainees

Award Recipients

2019

Kritika Chaddha

Jawaharlal Nehru University

Godwin Kwakye-Nuako

University of Cape Coast

\section{8}

Syed Mian

Jawaharlal Nehru University

2017

Rogger Carmen

Universidad Peruana Cayetano Heredia

2016

Emna Harigua Souiai

Institue Pasteur de Tunis, Tunisia

2015

Abel Nathan

Madurai Kamaraj University, India

\section{ACMCIP Award for Advanced Training}

Award Recipients

2019

Mary Lynn

University of South Carolina Arnold School of Public Health
2018

Ruwandi Kariyawasam

University of Toronto

2017

Camila Coelho

National Institute of Allergy and Infectious Diseases

Maria Luisa Simoes

Johns Hopkins University

2016

Vanessa Moraes

Oswaldo Cruz Foundation

2015

Heidi Hillesland

University of Washington

William Trager Award for Basic Parasitology Award Recipients

2019

David Horn

University of Dundee

2018

Elizabeth Winzeler

University of California, San Diego

2017

Boris Striepen

University of Pennsylvania

2016

Stefan Kappe

Center for Infectious

Disease Research

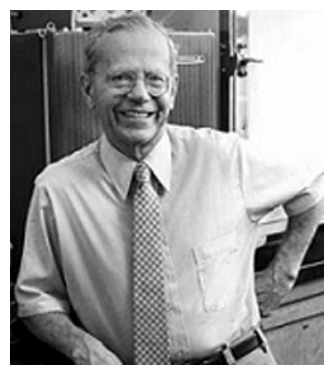




\section{CLINICAL GROUP}

\section{AMERICAN COMMITTEE ON CLINICAL TROPICAL MEDICINE AND TRAVELERS' HEALTH}

\section{Annual Business Meeting Minutes}

Thursday, November 21, 2019; 5:15-6:00 p.m. ASTMH 68th Annual Meeting

November 20-24, 2019

Gaylord National Resort and Convention Center

National Harbor, Maryland, USA

President: M. Patricia Joyce, MD

Secretary/Treasurer: Kristina Krohn, MD

1. The meeting was called to order by ACCTMTH President, Pat Joyce.

2. Elsevier-ASTMH Clinical Research Award winners (presented by Pat Joyce)

a. Awards are $1^{\text {st }}$ place $(\$ 500) ; 2^{\text {nd }}$ place $(\$ 300)$ and $3^{\text {rd }}$ place $(\$ 200)$ plus $\$ 200$ travel grant given to each presenter provided by the Clinical Group.

b. The Judges this year - David Kaminstein, Kristina Krohn. Latha Rajan, and John Sanders - were thanked.

d. 2019 Awardees

- First place, Titus Kwambai, Kenya Medical Research Unit, Kisumu, Kenya

- Second place, Melinda Tanabe, UTMB, Galveston, TX

- Third place, Ruwandi Kariyawasam, University Toronto, Canada

e. Thanks were given to Elsevier for their contributions

3. Election results. Past President David Brett-Major reviewed the 2019 election results:

a. Germán Henostoza was elected president-elect.

b. Dan Leung was elected to council for a 3-year term.

c. Thanks were offered to outgoing Councilor Janine Danko, Past-president David BrettMajor, and Student Representative Michael Harper.

4. Clinical Group finances were reviewed by Kristina Krohn, Secretary-Treasurer

a. Balance of fund include $\$ 167,115$ for the group plus $\$ 37,948$ for Marcolongo fund.

b. $\$ 10,000$ annual grant from parent society for 2018-2019 was completely spent, to include \$4016 fee for Marty S. Wolfe Mentoring Award medal and \$908 donation to AJTMH for clinical page charges.
5. Update on Educational Activities given by Brett HendelPatterson

a. 2018 CTropMed $^{\circledR}$ had 138 examinees with 83\% pass rate

b. 2019 Update Course held in Toronto in conjunction with North American Refugee Health Conference

c. Pre-Meeting Course held on "Tropical Diseases Encountered in Non-Travel Clinics"

d. Tropical Medicine and Global Health Exploratory Committee formulated to develop plan for Global Health-Tropical Medicine board certification.

6. New business was called for and completed.

7. Meeting Closure and Transfer of Authority

a. President (2018-2019) Pat Joyce transferred authority to the incoming President Latha Rajan (2019-2020) with the symbolic passing of the gavel.

b. President Latha Rajan closed the meeting. 


\section{ACCTMTH SUBGROUP \\ (AMERICAN COMMITTEE ON CLINICAL TROPICAL MEDICINE AND TRAVELERS' HEALTH)}

\begin{tabular}{|c|c|}
\hline INICAL GROUP LEADERSI & \\
\hline Member/Position & Term Expir \\
\hline Latha Rajan, President & .2020 \\
\hline Germán Henostroza, President-Elect & .2020 \\
\hline M. Patricia Joyce, Past-President. & 2020 \\
\hline Kristina Krohn, Secretary/Treasurer & . .2019 \\
\hline Natasha Hochberg, Councilor ..... . & .2020 \\
\hline Daniel Kaminstein, Councilor. & . .2021 \\
\hline Daniel Leung, Councilor. & . .2022 \\
\hline Austin Jones, Student Representativ & . . 2020 \\
\hline
\end{tabular}

\section{CLINICAL GROUP PAST LEADERSHIP}

Name Years

\section{President}

M. Patricia Joyce ....................... 2019

David Brett-Major ..................... 2018

John W. Sanders. . . . . . . . . . . . . . . . . . . . . . 2017

Duane Hospenthal . . . . . . . . . . . . . . . . . . . . . . 2016

Susan McLellan ........................ 2015

Richard Oberhelman .................... 2014

M. Patricia Joyce . . . . . . . . . . . . . . . . . . . . . 2013

David Hamer. . . . . . . . . . . . . . . . . . . . . . . . 2012

Philip Coyne .......................... 2011

William Stauffer . . . . . . . . . . . . . . . . . . . . . 2010

Joe Vinetz . . . . . . . . . . . . . . . . . . . . . . . . . . . . 2009

Alan Magill . . . . . . . . . . . . . . . . . . . . . . . . . . . 2008

Anne McCarthy . . . . . . . . . . . . . . . . . . . . 2007

J. Dick MacLean . . . . . . . . . . . . . . . . . . . . . . . . 2006

David Freedman. . . . . . . . . . . . . . . . . . . . . . 2005

Richard D. Pearson . . . . . . . . . . . . . . . . . . . 2002-2004

David R. Hill . . . . . . . . . . . . . . . . . . . . . . 2000-2002

A. Clinton White. . . . . . . . . . . . . . . . . . . . 1998-2000

Steve Hoffman . . . . . . . . . . . . . . . . . . . . . 1996-1998

Frank J. Bia . . . . . . . . . . . . . . . . . . . . . . . 1994-1996

Len Marcus. . . . . . . . . . . . . . . . . . . . . . . . . . . 1992-1994

Jay S. Keystone. . . . . . . . . . . . . . . . . . . . . . . . 1990-1992

Elaine Jong . . . . . . . . . . . . . . . . . . . . . . . . . . . 1988-1990

\section{Secretary-Treasurer}

Kristina Krohn. . . . . . . . . . . . . . . . . . . . . . . . 2019-

Latha Rajan. . . . . . . . . . . . . . . . . . . . . . . . . 2015-2018

Beth Kirkpatrick ....................... . 2012-2015

Paul Southern. . . . . . . . . . . . . . . . . . . . . . . . 2009-2012

M. Patricia Joyce ........ . . . . . . . . . . . . 2006-2009

Jan Evans Patterson . . . . . . . . . . . . . . . . . . 2003-2006

David R. Shlim. . . . . . . . . . . . . . . . . . . . . 2000-2003

Vernon Ansdell. . . . . . . . . . . . . . . . . . . . . 1997-2000
David Freedman. . . . . . . . . . . . . . . . . . . . . . . . . 1994-1997

Barbara Herwaldt. . . . . . . . . . . . . . . . . . . . . . 1991-1994

Murray Wittner . . . . . . . . . . . . . . . . . . . . . . . . 1988-1991

\section{Councilor}

Janine Danko . . . . . . . . . . . . . . . . . . . . 2017-2019

Miguel Cabada . . . . . . . . . . . . . . . . . . . . . . . . . . 2016-2018

Frederique Jacquerioz . . . . . . . . . . . . . . . . . . 2015-2017

Walter (Ted) Kuhn . . . . . . . . . . . . . . . . . . . 2014-2016

Brett Hendel-Paterson. . . . . . . . . . . . . . . . . . . 2013-2015

Michael Libman . . . . . . . . . . . . . . . . . . . . . . . . 2012-2014

Chandy John. . . . . . . . . . . . . . . . . . . . . . 2011-2013

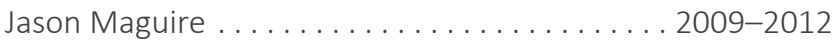

Elizabeth Barnett . . . . . . . . . . . . . . . . . . . . . . . . 2007-2010

Eric Houpt. . . . . . . . . . . . . . . . . . . . . . . . . . . . 2006-2009

Bradley Connor . . . . . . . . . . . . . . . . . . . . . 2005-2008

Alan Spira . . . . . . . . . . . . . . . . . . . . . . . . . . . . . 2004-2007

Philip E. Coyne, Jr. . . . . . . . . . . . . . . . . . . . . . . . 2003-2006

M. Patricia Joyce . . . . . . . . . . . . . . . . . . 2002-2005

Kenneth R. Dardick . . . . . . . . . . . . . . . . . . . . . . . 2003-2004

Claire Panosian. . . . . . . . . . . . . . . . . . . . . . . 2001-2004

Martin Cetron ......................... 2000-2003

Philip Fischer . . . . . . . . . . . . . . . . . . . 1999-2002

Monica Parise. . . . . . . . . . . . . . . . . . . . . . . . 1998-2001

Victor Kovner . . . . . . . . . . . . . . . . . . . . . . . 1998-2000

A. Clinton White. . . . . . . . . . . . . . . . . . . . . . 1997-1998

Douglas MacPherson. . . . . . . . . . . . . . . . . . . 1996-1999

Jan Evans Patterson . . . . . . . . . . . . . . . . . . . . . . 1995-1998

Thomas Strickland . . . . . . . . . . . . . . . . . . . . . 1994-1997

Phyllis Kozarsky . . . . . . . . . . . . . . . . . . . . . . . . . 1994-1996

Frank Bia . . . . . . . . . . . . . . . . . . . . . . . . . . . 1993-1994

David Hill. . . . . . . . . . . . . . . . . . . . . . . . . . . . . . 1992-1995

Marty Wolfe . . . . . . . . . . . . . . . . . . . . . . . . . 1991-1994

Ralph Bryan . . . . . . . . . . . . . . . . . . . . . . . 1990-1993

Len Marcus. . . . . . . . . . . . . . . . . . . . . . . . . 1989-1992

Bob Goldsmith. . . . . . . . . . . . . . . . . . . . 1989-1991

Michele Barry. . . . . . . . . . . . . . . . . . . . . . . 1989-1990 
CLINICAL GROUP

\section{AMERICAN COMMITTEE ON CLINICAL TROPICAL MEDICINE AND TRAVELERS' HEALTH}

\section{CONSTITUTION AND BYLAWS OF THE CLINICAL GROUP \\ ARTICLE I \\ NAME AND PURPOSE \\ Section 1}

This organization shall be called the American Committee on Clinical Tropical Medicine and Travelers' Health (ACCTMTH). In most instances, it will be referred to as the "Clinical Group."

Section 2

The purposes of this organization shall be to promote the Clinical aspects of tropical medicine, medical parasitology and travelers' health.

\section{ARTICLE II \\ MEMBERSHIP \\ Section 1 \\ There shall be three classes of members: active, honorary and student.}

Section 2

Active members will be those individuals who are members of the American Society of Tropical Medicine and Hygiene (ASTMH), who are actively engaged in, or interested in, clinical aspects of tropical medicine, medical parasitology and/or travelers' health.

\section{Section 3}

Students within the biomedical sciences who have an interest in clinical areas of tropical medicine, medical parasitology, and/or travelers' health may become student members. They shall have all the privileges of membership except voting and holding office.

\section{Section 4}

Honorary members shall be nominated by a two-thirds vote of the council and approved by at least two-thirds of members' casting votes. Honorary members will be individuals outside this organization recognized for their outstanding contributions to clinical tropical medicine and travelers' health. They shall have all the privileges of membership except voting and holding office.

\section{ARTICLE III \\ OFFICERS \\ Section 1 \\ The officers of the organization shall be a President-Elect, President, and Past President, Secretary/Treasurer and three \\ Councilors. Any active member in good standing is eligible for election to office. The officers shall constitute the council, in which the government of the organization shall be vested.}

The President-Elect, President and Past President shall each serve terms of one year in succession. The Secretary/Treasurer and the Councilors will be elected for a term of three years. Terms of office in the organization shall begin at the close of the annual meeting at which the officers were declared elected. Officers may run for and serve consecutive terms.

If the presidency becomes vacant, the President-Elect shall become the President for the remainder of the unexpired term. The President may fill vacancies among the Councilors by appointing Councilors from the active membership to serve until the missing Councilor returns to office or through the remainder of the absent Councilor's term, if return to office is not possible. If the Secretary/ Treasurer is unable to complete his/her term, the President will appoint a replacement from the active membership or Councilors to serve until the next annual meeting when a new Secretary/ Treasurer will be elected.

Section 2

The duties of the President shall be those regularly devolving on the chief executive officer. $\mathrm{He} /$ she shall preside at subcommittees as provided in the bylaws.

Section 3

The Secretary/Treasurer and/or Secretariat of the ASTMH shall be the custodian of all records of the Committee. They shall handle the business and finances of the committee and shall submit an annual financial report.

The Secretary/Treasurer and/or Secretariat of the ASTMH shall be responsible for mailings to members, including mailing of ballots and other business matters. The Secretary/ Treasurer shall preside over the Committee in the absence of the President.

Section 4

Council shall annually appoint a nominating subcommittee consisting of five active members. No more than three of those members may be current members of Council. A slate of candidates for offices to be filled at the next annual meeting shall be submitted by mail and/or electronic mail no later than two months prior to the next annual meeting. Members may submit themselves or suggest other active members as candidates to the Chairman of the nominating committee, but the nominating committee will not be obligated to put such individual(s) on the slate unless there is a supporting petition from three active members in addition to the individual proposed. 
Election for each office shall be by secret mail and/or electronic mail ballot and determined by majority vote (or by the greatest number of votes if more than two candidates). Mailing and counting ballots shall be the responsibility of the Secretary/Treasurer and/or Secretariat of the ASTMH. The results of voting will be announced in a Newsletter and at the next annual meeting.

\section{ARTICLE IV}

\section{AMENDMENT OF CONSTITUTION}

Section 1

Amendments to this constitution and bylaws may be adopted on approval by a majority of the ACCTMTH council and upon a two-thirds affirmative vote of ballots cast subject to the following conditions:

The proposed amendment must be in writing and signed by at least three members of the Committee.

The signed proposed amendment must be in the hands of the Secretary/Treasurer and/or Secretariat of the ASTMH before the annual meeting of the Committee.

Voting of proposed amendments by the active members will be done by mail and/or electronic mail, except as provided in section $1 \mathrm{~d}$, below. The mailing and counting of ballots shall be the responsibility of the Secretary/Treasurer and/or Secretariat of the ASTMH. Results of balloting will be announced at or before the annual meeting following the vote.

If at least 25 percent of active members are present at an annual meeting, a majority of those present can require a vote on a Council-approved amendment at that meeting. The amendment can be passed by two-thirds of ballots cast.

\section{ARTICLE V}

COUNCIL FUNCTION

The Council shall manage the Committee's affairs and direct its business. It shall initiate, coordinate and terminate the work of the subcommittees. It shall have the power to approve the expenditure of funds and establish an annual budget for the Committee.

\section{ARTICLE VI}

\section{MEETINGS}

The Committee shall meet annually for business and scientific sessions. The business meeting and at least one scientific meeting will be held in conjunction with the annual meeting of the ASTMH.

In addition, Council may call for other scientific sessions for purposes of continuing medical education. Council will determine the time and place of such additional meetings.

In the conduct of business sessions, Robert's Rules of Order shall be the guide. At the beginning of each session, the President shall appoint a member to serve as parliamentarian. Minutes will be recorded and maintained by the Secretary/Treasurer.

\section{ARTICLE VII SUBCOMMITTEES}

The President shall appoint annually a Program Chairman who should work cooperatively with the program committee of the ASTMH. With approval by a majority of council members, the President may create and appoint ad hoc subcommittees, as needed.

\section{ARTICLE VIII}

DUES

Dues may be assessed and changed by a majority vote of the ACCTMTH Council, subject to approval by a majority of active members voting. A quorum of twenty percent of active members may be called and required for that vote.

\section{ARTICLE IX}

\section{ADVISORY SUBCOMMITTEES}

The Past Presidents of this organization will collectively form an advisory subcommittee. This subcommittee may bring matters to the attention of Council and advise it on matters it is considering. However, neither the advisory subcommittee nor its members, except for the immediate Past President, will have a vote in Council affairs, unless the member has been re-elected as an officer of ACCTMTH.

\section{AMENDMENT 1 (18 NOV, 1992)}

A condition of active membership will be payment of dues.

\section{AMENDMENT 2 (30 NOV, 1992)}

Council shall annually appoint a nominating subcommittee consisting of five active members. No more than three of those members may be current members of Council. A slate of candidates for offices to be filled at the next annual meeting shall be submitted by mail and/or electronic mail no later than two months prior to the next annual meeting. Members may submit themselves or suggest other active members as candidates to the Chairman of the nominating committee, but the nominating committee will not be obligated to put such individual(s) on the slate unless there is a supporting petition from three active members in addition to the individual proposed.

Election for each office shall be by secret mail and/or electronic mail ballot and determined by majority vote. Mailing and counting ballots shall be the responsibility of the Secretary/Treasurer and/or Secretariat of the ASTMH. The results of voting will be announced in a Newsletter and at the next annual meeting.

Revised: 8 October 2004 

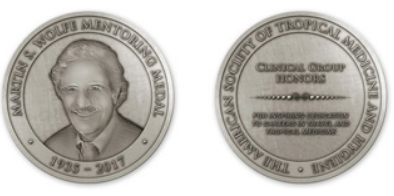

\section{Awards}

Martin S. Wolfe Mentoring Award-

The Clinical Group has established an award to honor the life of inspiring mentorship by our friend, teacher and colleague, Martin S. Wolfe, MD, FACP, FASTMH. This award will recognize individuals who have served as exemplary and inspiring mentors. It will be presented to a member of the American Committee on Clinical Tropical Medicine and Travelers' Health (ACCTMTH, the Clinical Group) who has been exceptional in guiding the professional growth of careers in tropical and travel medicine.

\section{Recipient}

Elaine Jong

University of Washington School of Medicine

\section{Elsevier Clinical Research Award- \\ Recognizes excellence in research presented by a student at the Annual Meeting.}

2019

Titus Kwambai

Melinda Tanabe

Ruwandi Kariyawasan

2018

Neima Briggs

Jonathan Chang

Thomas Siegert

2017

Inke Lubis

Menno Smit

Charlotte Heuvelings

\section{6}

Edward Smith

Ruwandi Kariyawasan

Obadia Kenji

\section{5}

Rahajeng N. Tunjungputri

Sarah Boudova

Ross Boyce

\section{4}

Paul Griffin

Junxiong Pang

Luis Marcos
2013

Remko Schats

Sara-Blythe Ballard

Samuel Tassi Yunga

2012

Else Bijker

Grace Chan

Jesica Christensen

2011

Andrew Brent

Elizabeth Schlaudecker

Luther Bartelt

2010

Paul Krezanoski

Kevin Esch

Jennifer Downs

\section{Elsevier Student Book Award- \\ Recognizes excellence in research presented by a student at the annual meeting.}

2009

An Na Park

2008

Eugene Richardson

2007

Aglaêr Alves da Nóbrega

2006

Sarah Landis

2005

Alisa Alker

2004

Luis Marcos Raymundo

2003

Jeffrey K. Eng

2002

John Todd Utz

2001

Tina Marie Loane Peterson

2001

Kai-Hsin Chang

2000

Shingairai Feresu

1999

Daniel Grushka 


\section{THE ASTMH COMMITTEE ON GLOBAL HEALTH (ACGH)}

\section{ASTMH Committee on Global Health Business \\ Meeting 2019}

Date: November 21, 2019

Room: Potomac Ballroom A, Ballroom Floor

Location: Gaylord Resort National Harbor, MD

\section{Agenda - Items discussed}

1. Introduction (Julie)

2. President's report

a. Registered members: 942, which includes 320 regular members, 311 students, and 275 postdocs.

3. Financial Report (Miguel)

a. Council Designated Funds: According to the final statement, the starting balance for the fiscal year 2019 (July 1st, 2018 to June 30th, 2019) was 23,710 USD. Total income for the fiscal year was 12,518 USD (mostly from Global Health Dues, 10,205 USD). The only expense was the e-ballot, which cost $\$ 659.00$. End balance (as of June 30 th, 2019) is $\$ 35,637.00$. Please note that this statement is pre-audit and may change after audit.

b. 10K Yearly Pilot Fund (must be used within fiscal year). Total income $\$ 10,000$. Total expenses $\$ 10,000.00$, as follows: $\$ 2,748.38$ for expenses related to $\mathrm{ACGH}$-sponsored sessions in the annual meeting; \$5,500.00 in Travel Awards; \$1,491.25 in Symposium Speakers; and, 260.37 used to partially support Research Awards. Income was to be used within this fiscal year, which is now complete.

c. Future discussion topic: What should we be spending our funds on for 2020?

4. Election results: newly elected officers: Miguel Reina Ortiz (President elect), Eri Togami (Secretary \& treasurer), Katie Anderson and David Gittelman (Councilors).

5. New leadership (as of Nov 21, 2019): Robert Newman (President), Miguel Reina-Ortiz (President-elect), Julie Pavlin (Past president), Eri Togami (Secretary \& treasurer), Joel Montgomery, Ilin Chuang, Katie Anderson, David Gittelman (Councilors), Joanne Gbenjo (Student/trainee representative)

6. Awards:

a. Travel award: Saikou Bah (Ghana), Caleb Stica (Tanzania). Selected from 25 applications.

b. Student award: Rachel Bensman (Cincinnati, $\mathrm{OH}$ ), Daniele Gusland (Madison, WI), Andrea Perez (Guatemala). Selected from 42 applications.

i. Student award was very well received. ACGH should discuss continuing the award.

7. Andrea Perez' research (student award recipient)

a. Currently undergraduate student in Guatemala

b. Overview of research i. Evaluation of genetic structure of four strains of Trypanosoma cruzii for endemic areas in Guatemala, including border zone with Mexico, using mouse biological model. Study found genetic differences of parasites do correlate with severity of patient symptoms.

8. 2019 Annual Meeting activities - thank you to all organizers

a. Pre-Meeting Course: Field Applications for Stopping Infectious Disease Threats at International Points of Entry: Co-chairs were Clive Brown and Miguel Reina Ortiz.

b. Symposium I: Creating a Sustainable Business for Global Health Innovations: Organizers were Robert Newman and Ilin Chuang.

c. Symposium II: Diverse Pathogens, Common Risk Factor: Infections of Poverty in the United States. Organizers were Eileen Stillwagon, and Jessica Manning.

d. Ponder-to-Probe: A Cosmopolitan Debate and Peer Networking. Organizers were Koya Allen, Latasha Allen, and Charlotte Hobbs.

e. Speed-networking with the Experts. Organizers were Koya Allen, Stephen Fischer, and Jessica Manning. This activity will be taken over by student committee.

f. Moving Back Home: Strategies for Returning Back to LMICs after Training Abroad. Co-chairs were Johanna Daily, Abiola Fasina, Ayaman Ahmed, Linnie Golightly

9. Communications - ACGH routinely communicates to its members through newsletters, please read them.

10. Hand-over of Presidency from Julie Pavlin to Robert Newman

11. New President's remarks (Robert Newman)

a. Thank you Julie for excellent leadership in the past year. Julie will still stay engaged as past president.

b. Awarding certificates of appreciation

i. Travel award: Caleb and Saikou

ii. Outgoing Secretary Treasurer: Miguel Reina Ortiz

iii. Outgoing past-president: Ramin Asgary

iv. Outgoing Councilors: Jessica Manning and Eileen Stillwaggon

v. Outgoing President: Julie Pavlin

c. ACGH council meeting: took place yesterday (Nov 20, 4:30-6:30pm) - great discussion about strategic plans, planning activities for upcoming pre-meeting course, symposia, etc.

i. Please provide feedback in the next two weeks for ACGH's strategic plan.

d. Providing membership

i. There are approx. 950 ACGH members, two thirds of whom are trainees. ACGH will focus 


\section{THE ASTMH COMMITTEE ON GLOBAL HEALTH (ACGH)}

on mobilization of mentors to encourage engagement of these younger members and provide useful information on leadership and management.

ii. One-time mentorship events: very good, continue activities

iii. Longitudinal mentorship: very important. ACGH will call for mentors and match them with trainees, with a minimum year-long (remote) commitment.

12. Meeting adjourned

\section{Leadership Member/Position} Term Expires

Robert Newman, President . . . . . . . . . . . . . . 2020 Miguel Reina Ortiz, President-Elect. . . . . . . . . . 2020 Julie Pavlin, Past-President. . . . . . . . . . . . . . 2020 Eri Togami, Secretary/Treasurer . . . . . . . . . . . 2021 Ilin Chuang, Councilor. . . . . . . . . . . . . . . . . . . 2020 Joel Montgomery, Councilor . . . . . . . . . . . . . . 2020 Kathryn Anderson, Councilor . . . . . . . . . . . . . . 2021 David Gittelman, Councilor . . . . . . . . . . . . . . . . 2021 Joanne Gbenjo, Student Representative . . . . . . . 2020

\section{CHARTER OF THE ASTMH COMMITTEE ON GLOBAL HEALTH}

\section{Objectives}

- The ASTMH Committee on Global Health (ACGH) is organized under the auspices of the American Society of Tropical Medicine and Hygiene (ASTMH) and has the following objectives:

- To promote the development of the field of Global Health which addresses multidisciplinary transnational approaches to health issues that unfavorably affect underserved and under-resourced populations.

- To educate about global health issues inclusive of and in addition to ASTMH's traditional purview of tropical medicine and hygiene through pre-courses, workshops, symposia and abstract presentations

- To foster collaborations between individuals and groups that address global health issues

- To facilitate dissemination and publication of global health research

- To provide recruitment opportunities for individuals interested in pursuing a career in global health.

- To serve as a representative voice from ASTMH in other Global Health organizations, societies and working groups.

- To recognize outstanding contributions in Global
Health

\section{Meetings}

The ACGH will meet at least annually under the aegis of the ASTMH Annual Meeting.

\section{Membership}

Members in good standing of ASTMH who wish to join ACGH may do so by paying subgroup dues in accordance with rates set by the ACGH Executive Council. ACGH will establish a fund for voluntary donations to be managed by ASTMH. Funds collected on behalf of ACGH from sources such as dues, voluntary donations, etc. will be managed by ASTMH and used for activities that further the objectives of ACGH, including receptions, annual awards, fellowships and international training opportunities, and payment of page charges in the American Journal of Tropical Medicine and Hygiene for qualifying articles (e.g. editorials, topical reviews, symposium proceedings, cutting-edge papers, etc.).

\section{Governance}

\section{Executive Council}

There will be elected leadership in the form of an Executive Council consisting of a President, President-Elect, Past President, Secretary- Treasurer and four Councilors. Any active member in goodstanding is eligible for election to office. The initial leadership of the Committee on Global Health will serve for a total of 18 months.

The President-Elect and President will each serve terms of one year in succession. The Secretary-Treasurer and Councilors serve terms of two years. Terms of office begin at the close of the annual meeting at which the officers were declared elected. All members of the ACGH Executive Council are allowed to vote on Council business. Council members may run for and serve consecutive terms.

The President serves as chief executive officer for the ACGH and presides over meetings of the ACGHExecutive Council and the ACGH annual business meeting. The President may fill vacancies among the Councilors by appointing Councilors from the membership to serve until the missing Councilor returns to office or through the remainder of the absent Councilor's term, if return to office is not possible. If the Secretary-Treasurer is unable to complete his/her term, the President will appoint a replacement from the membership to serve until the next annual meeting when a new SecretaryTreasurer will be elected.

The President-Elect presides over meetings of the ACGH in 
the absence of the President. If the presidency becomes vacant, the President-Elect will serve out the balance of the President's unexpired term before going on to complete his/ her originally- scheduled term as President.

The Secretary-Treasurer of the ACGH works with the Secretary- Treasurer of ASTMH to monitor the Committee's finances and to develop its annual budget. The ACGH Secretary-Treasurer, ACGH President, or person determined by the ACGH President maintains minutes of the ACGH Executive Committee and annual business meeting and compiles reports on ACGH activities for presentation to the ASTMH Council as requested.

\section{Elections}

The ACGH Executive Council appoints a nominating subcommittee consisting of five active members. No more than three of those members may be current members of the ACGH Executive Council. A slate of candidates for offices to be filled at the next annual meeting will be distributed to ACGH members by mail and/or electronic mail no later than two months prior to the next annual meeting. Members may submit themselves or suggest other active members as candidates to the Chairman of the nominating committee, but the nominating committee will not be obligated to put such individual(s) on the slate unless there is a supporting petition from three active members in addition to the individual proposed.

Election for each office shall be by secret mail and/or electronic mail ballot and determined by majority vote (or by the greatest number of votes if more than two candidates). Results of voting will be announced in the ASTMH newsletter and at the next ACGH annual business meeting.

\section{Subcommittees}

Subcommittees may be formed to deal with important issues such as awards, pre-course development, fundraising, career opportunities, interfacing with the American Journal of Tropical Medicine and Hygiene, and planning of ACGH-supported symposia and workshops at the ASTMH annual meeting. Members of the ACGH are encouraged to apply to participate on the Global Health Scientific Program Committee, following Society guidelines for participating on the Scientific Program Committee.

\section{Awards}

\section{ACGH Award for Research Support}

\section{Recipients}

2019

Rachel Bensman
Cincinnati Children's Hospital Medical Center

Daniele Gusland

University of Wisconsin

Andrea Perez

Universidad de San Carlos de Guatemala

\section{ACGH Student Travel Award}

\section{Recipients}

2019

Saikou Bah

WACCBIP

Caleb Stica

Ifakara Health Institute

\section{8}

Sarah Boudova

Indiana University

Mahfuza Talukder Flowra

Universitas Gadjah Mada

\section{7}

Meredith Hickson

University of Michigan Medical School

Martha Tesfalul

University of California San Francisco

\section{6}

E. Ross Colgate

University of Vermont

Louisa Messenger

London School of Hygiene \& Tropical Medicine

\section{5}

Rebecca Fischer

Baylor College of Medicine, National School of Tropical

Medicine

Marie Nancy Seraphin

University of Florida

\section{4}

Nathan Lo

Stanford University School of Medicine

Elizabeth Thiele

Centers for Disease Control and Prevention 